\title{
Principal Unconformities in Triassic and Jurassic Rocks, Western Interior United States- A Preliminary Survey
}

GEOLOGICAL SURVEY PROFESIONAL PAPER 1035-A

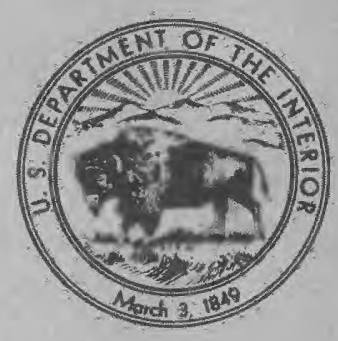





\section{Principal Unconformities in Triassic and Jurassic Rocks, Western Interior United States- A Preliminary Survey}

By G. N. PIPIRINGOS and R. B. O'SULLIVAN

UNCONFORMITIES, CORRELATION, AND NOMENCLATURE OF SOME TRIASSIC AND JURASSIC ROCKS, WESTERN INTERIOR UNITED STATES

GEOLOGICAL SURVEY PROFESSIONAL PAPER 1035-A

Description of nine widespread unconformities in Triassic and Jurassic rocks

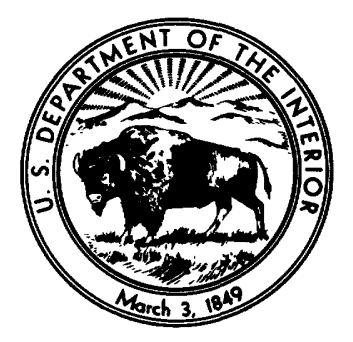




\title{
UNITED STATES DEPARTMENT OF THE INTERIOR
}

\author{
CECIL D. ANDRUS, Secretary
}

\section{GEOLOGICAL SURVEY}

H. William Menard, Director

\footnotetext{
Library of Congress Cataloging in Publication Data

Pipiringos, Geórge Nicholas, 1918

Principal unconformities in Triassic and Jurassic rocks, Western Interior United States.

(Unconformities, correlation, and nomenclature of some Triassic and Jurassic rocks, Western Interior United States)

(Geological Survey Professional Paper 1035-A)

Bibliography: p. 26

1. Geology, Stratigraphic-Triassic. 2. Geology, Stratigraphic-Jurassic. 3. Stratigraphic correlation-The West.

4. Geology-The West. I. O'Sullivan, Robert Brett, 1923-joint author. II. Title. III. Series. IV. Series:

United States Geological Survey Professional Paper 1035-A

QE676.P5 $551.7^{\prime} 6^{\prime} 0978$ $77-608355$
}

For sale by the Superintendent of Documents, U.S. Government Printing Office

Washington, D. C. 20402

Stock Number 024-001-03125-6 


\section{CONTENTS}

\begin{tabular}{|c|c|}
\hline Page & Page \\
\hline 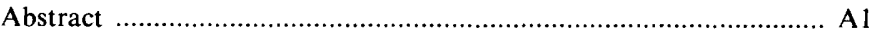 & Jurassic unconformities \\
\hline Introduction & 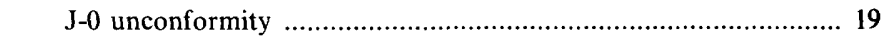 \\
\hline Previous work & 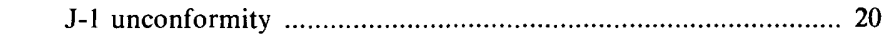 \\
\hline 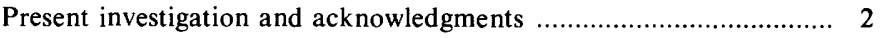 & $\mathrm{J}-2$ unconformity . \\
\hline Unconf ormities & $\mathrm{J}-3$ unconformity ......... \\
\hline 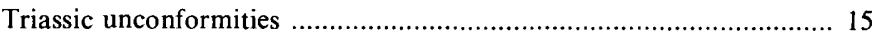 & 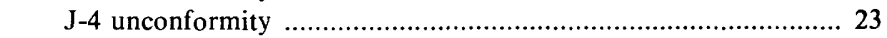 \\
\hline 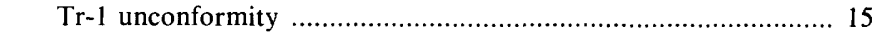 & J-5 unconformity .......... \\
\hline 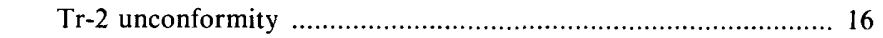 & Cretaceous unconformity ..... \\
\hline 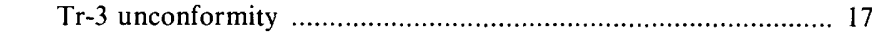 & 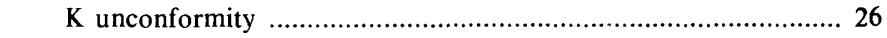 \\
\hline 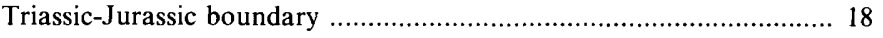 & 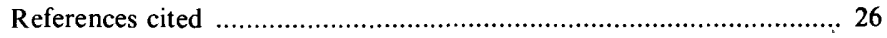 \\
\hline
\end{tabular}

\section{ILLUSTRATIONS}

Plate 1. Restored sections showing principal unconformities in Triassic rocks in Western Interior United States and paleogeologic maps of the J-2 unconformity In pocket

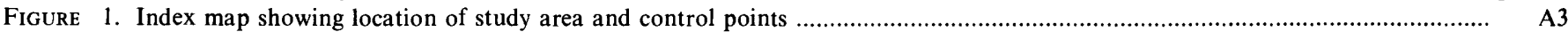

2. Diagram illustrating the arrangement and relationships of the several unconformities to each other and to the enclosed sedimentary wedges

3. Diagram illustrating two corollaries of the law of superposition

4. Diagram illustrating relationship of unconformities to a paleo-high

5. Series, stages, and time-scales of the Jurassic and Triassic Systems used in this report

\section{TABLES}

TABLE 1. Location of control points and source of data used to construct restored sections and maps in this report

2. Lithologic descriptions of stratigraphic units

Page

A4 



\title{
PRINCIPAL UNCONFORMITIES IN TRIASSIC AND JURASSIC ROCKS, WESTERN INTERIOR UNITED STATES-A PRELIMINARY SURVEY
}

\author{
By George N. Pipiringos and Robert B. O’Sullivan
}

\begin{abstract}
The Triassic and Jurassic rocks in Western Interior United States contain nine unconformities each of which was destroyed to some extent by a younger unconformity. Regardless of extent, all are useful for correlation of rock sequences in areas where fossils or age dates are lacking. The purpose of this report is to call attention to the presence, significance, and value for correlation of these unconformities. The Triassic unconformities are designated from oldest to youngest, $\mathrm{Tr}-1, \mathrm{Tr}-2$, and $\mathrm{Tr}-3$; the Jurassic ones similarly are designated $\mathrm{J}-0, \mathrm{~J}-1, \mathrm{~J}-2, \mathrm{~J}-3, \mathrm{~J}-4$, and $\mathrm{J}-5$. Of these, the $\mathrm{J}-2$ surface is the best preserved and most widespread. It extends throughout the Western Interior and truncates the older unconformities in different parts of this area. Consequently, the $\mathrm{J}-2$ surface is discussed and illustrated in much more detail than the others.
\end{abstract}

Identification of these unconformities throughout large areas where their presence hitherto had been unknown results in some new unexpected correlations and conclusions. Principal among these are: (1) The Red Draw Member of the Jelm Formation of southeastern Wyoming equals the lower part of the Crow Mountain Sandstone of central Wyoming. The Sips Creek Member of the Jelm Formation of southeastern Wyoming equals the upper part of the Crow Mountain Sandstone of central Wyoming and the Gartra Member of the Chinle Formation in the Uinta Mountains of northeastern Utah and northwestern Colorado. The Chinle Formation of the Colorado Plateau and the Uinta Mountains equals the upper part of the Crow Mountain plus the Popo Agie Formation of central Wyoming. (2) The Nugget Sandstone of northern Utah and southwestern Wyoming approximately equals the Glen Canyon Group of the Colorado Plateau. The Temple Cap Sandstone of southwestern Utah equals the Gypsum Spring Formation and the Gypsum Spring Member of the Twin Creek Limestone of Wyoming and the Nesson Formation of Nordquist in the subsurface of the Williston basin. The Sawtooth and Piper Formations at their type sections in Montana and the lower parts of the Twin Creek Limestone (including only the Sliderock, Rich, and Boundary Ridge Members) in western Wyoming and of the Carmel Formation in the Colorado Plateau, at their respective type localities, are equivalent, but none of these correlate with any part of the Gypsum Spring Formation of Wyoming. The Curtis Formation at its type locality in the San Rafael Swell, Utah, equals only the lower part of the Curtis Formation of the Uinta Mountains. The upper part of the Curtis in the Uinta Mountains and the Redwater Shale Member of the Sundance Formation of Wyoming and South Dakota are equivalent.

Estimates of the length of time in millions of years (m.y.) required for uplift and erosion of an unconformity range from less than 1 to as much as $10 \mathrm{~m} . \mathrm{y}$.; the average is about $1.8 \mathrm{~m} . \mathrm{y}$. if the extremes in time are excluded. The length of time for burial of the surfaces by transgression ranges from less than 1 to about 10 m.y.; the average is less than 1 m.y. if the extremes in time are disregarded.

\section{INTRODUCTION}

The Triassic and Jurassic rocks of the Western Interior were deposited on a stable shelf that sloped mainly westward in Triassic time and mainly northwestward in Jurassic time. Deposition was interrupted several times by epeiric uplift and subsequent erosion. Each erosion surface was then preserved by burial beneath the initial deposits of transgressive seas or by continental filling of subsiding basins.

In the Western Interior, rocks of Triassic and Jurassic age contain nine widespread unconformities or erosional surfaces. The Triassic unconformities are designated from oldest to youngest, $\operatorname{Tr}-1, \operatorname{Tr}-2, \operatorname{Tr}-3$; the Jurassic unconformities similarly are designated $\mathrm{J}-0, \mathrm{~J}-1, \mathrm{~J}-2, \mathrm{~J}-3, \mathrm{~J}-4, \mathrm{~J}-5$. Of these, the $\mathrm{J}-2$ surface is the best preserved and most extensive. 
Some of these surfaces were destroyed in large part by erosion that produced later surfaces, some have remained untouched throughout most of the area, and some originally were less widespread than others. All, regardless of areal extent, are eminently useful for setting physical limits that cannot be transcended by correlations based on fossils or on absolute age determinations. In areas where fossils or age dates are lacking, the unconformities are essential for establishing correlations. These unconformities are also of potential economic significance in the search for petroleum and for some mineral deposits of sedimentary origin. Evaluation of the economic potential of these ancient surfaces of erosion, however, is not attempted in this report.

The purpose of this report is to call attention to the presence, significance, and value for correlation of the principal unconformities in the Triassic and Jurassic rocks of the Western Interior. An index map (fig. 1) shows the location of the study area, control points used in this report, the lines of sections, and present-day outcrops of Precambrian rocks. The stratigraphic position and areal distribution of the unconformities are shown in five restored sections (pl. 1). The $\mathrm{J}-2$ unconformity is further portrayed by means of two paleogeologic maps (maps $A$ and $B$, pl. 1) and is discussed in more detail than the others because it is the most widespread, the most easily detected, and the best documented of all the unconformities considered here. The location of control points and the source of data used to construct the restored sections and maps are listed in table 1. Throughout the text a letter and number in brackets following a place name refers to a locality described in table 1 and shown on the restored sections and maps. The relation of the stratigraphic units to each other and to the unconformities is shown on plate 1 . The stratigraphic units used in this report are also listed and briefly described in table 2 .

A subordinate objective of this report is to introduce a numerical system of nomenclature to designate, and thereby facilitate discussion of, each widespread unconformity in the Triassic and Jurassic rocks of the Western Interior. The numbering of the unconformities is portrayed in figure 2 , and is also shown on plate 1 .

\section{PREVIOUS WORK}

Some of the unconformities discussed herein have been recognized locally by previous workers, but none has been portrayed in its entirety except the unconformity at the base of the Moenkopi $(\mathrm{Tr}-1)$. Some comprehensive reports on the stratigraphy of Triassic and Jurassic rocks (McKee and others, 1956, pl. 2; McKee and others, 1959, pl. 2; MacLachlan, 1972, fig. 2; J. A. Peterson, 1972, pl. 2; and Stewart, Poole, and Wilson, 1972a, b) show, by means of maps, the lithofacies and thicknesses of arbitrarily chosen rock sequences, but they are only incidentally concerned with erosional surfaces. Other comprehensive reports, such as by Reeside and others (1957) and Imlay (1952a), are principally concerned with the segregation of rock sequences by fossil assemblages; physical breaks in the depositional record are mentioned only in passing - their significance in correlation is not discussed. Some of the unconformities discussed in this report either were not recognized in the foregoing reports or were misidentified. We have not made an exhaustive search of the literature for references to the unconformities except for reports covering large regions; many pertinent reports, however, have been examined and are cited at appropriate places in the text.

\section{PRESENT INVESTIGATIONS AND ACKNOWLEDGMENTS}

This report brings together some of the results of two independent stratigraphic investigations of Triassic and Jurassic rocks. These investigations were carried out by $\mathbf{G}$. N. Pipringos in southern Wyoming, northeastern Utah, and northwestern Colorado from 1958 to 1964, and by R. B. O'Sullivan in the Four Corners area from 1956 to 1963. Some results of these investigations were described by Pipiringos (U.S. Geol. Survey, 1965, p. A88; 1967; 1968; 1972, p. 18-29); Pipiringos, Hail, and Izett (1969); Pipiringos and O'Sullivan (1975, 1976); O'Sullivan (1965, 1970, 1974); O'Sullivan and Beikman (1963); O'Sullivan and others (1972); O'Sullivan and Green (1973); and O'Sullivan and Craig (1973).

In 1964, Pipiringos accompanied Ralph W. Imlay of the U.S. Geological Survey on a tour of all the type sections of Jurassic rock-stratigraphic units in Montana, Idaho, Wyoming, and northern Utah. In early September of the same year, Pipiringos examined all the type sections of the various members of the Sundance Formation (Upper and Middle Jurassic) in the Black Hills of Wyoming and South Dakota. Still later that fall, Pipiringos and O'Sullivan visited nearly all the type sections of Triassic and Jurassic rock-stratigraphic units in the Colorado Plateau, including the Wingate-Thoreau area of New Mexico [NM15 and NM16]. By that time it was obvious (Pipiringos 1968, p. D19; U.S. Geological Survey, 1965) that the J-2 surface of unconformity extended from Buckhorn Wash [U12], in the San Rafael Swell (fig. 1), central Utah, eastward to Ralston Reservoir [C32], near Denver, Colo., and from Zuni, N. Mex. [NM20], at least as far north as Redwater Creek [SD1] in the Black Hills, S. Dak.

Other brief field trips or visits, in various widely scattered parts of the Western Interior, with members of the U.S. Geological Survey B. H. Bryant, D. D. Dickey, G. A. Izett, and F. G. Poole in 1964, D. M. Kinney, M. W. Reynolds, and G. L. Snyder in 1965; C. S. Bromfield, R. B. Johnson, E. V. Stephens, and H. D. Zeller in 1966; N. M. Denson, R. W. Imlay, J. F. Smith, Jr., and J. C. Wright in 1967; N. M. Denson in 1969; R. A. Cadigan, L. C. Craig, M. W. Green, Fred Peterson, and J. D. Strobell, Jr., in 1970; and M. W. Green in 1971, gradually filled in many gaps in our 


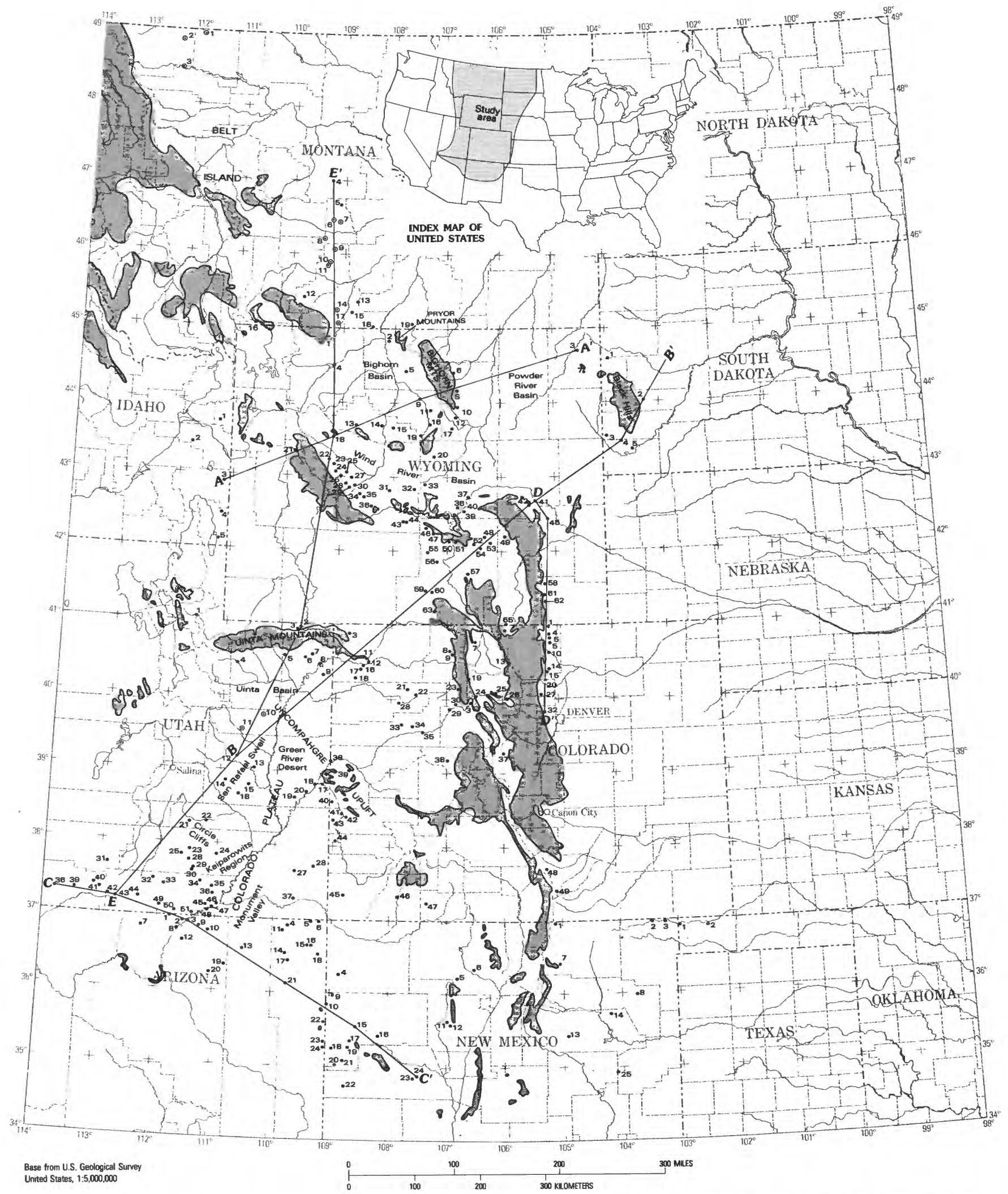

FIGURE 1.-Index map showing location of study area, control points used to construct cross sections $A-A^{\prime}$ through $E-E$ and paleogeologic maps on plate 1 , and present-day outcrops of Precambrian rocks (shaded). Control points are listed in table 1 by State and number. 
knowledge of unconformities in general and the distribution of the chert pebbles associated with the J-2 surface of erosion in particular. W. I. Finch contributed samples of conglomerate and color slides of an angular unconformity separating the Entrada Sandstone from underlying Triassic rocks, demonstrating that the $\mathrm{J}-2$ surface extends into the Panhandle of Oklahoma [Ok1 and Ok2]. H. D. Zeller contributed samples of conglomerate and color slides of the J-2 surface at Seep Flat, Utah [U30] (Pipiringos and O'Sullivan, 1975, figs. $5 E$ and $5 F$ ).

Fred Peterson identified the J-2 unconformity about $55 \mathrm{~m}$ below what was then considered the top of the Navajo Sandstone at Page, Ariz. [A3], in 1970, and traced it westward as far as Gunlock, Utah [U38]. Peterson established, in addition to the $\mathrm{J}-2$ surface, the presence of the $\mathrm{J}-3$ and the J-5 unconformities in a roughly triangular area with Garnet Ridge [A4] at the eastern apex, Fremont River [U22] at the northern apex, and Gunlock [U38] at the western apex. Some of the results of his work are given in Peterson (1973), Peterson and Barnum (1973a, b), and Peterson and Pipiringos (1978). Peterson furnished us some unpublished data that are credited in table 1 . Morris W. Green traced the $\mathrm{J}-2$ surface eastward from Thoreau, N. Mex. [NM16] nearly to the eastern New Mexico State line [NM8], and southeastward to near Acoma [NM23]. These and other data that he has contributed are credited in table 1. Some of these results appeared in Green (1974).

Several members of the U.S. Geological Survey have contributed J-2 chert-pebble collections or have furnished other stratigraphic information; their help is acknowledged and cited in table 1. Special thanks are due Ralph W. Imlay for taking the time to help Pipiringos hunt J-2 chert pebbles in the Black Hills of Wyoming and South Dakota in 1966, and in the Bighorn Mountains and Bighorn Basin, Wyo., in 1971. His unflagging enthusiasm and encouragement contributed immeasurably to the success of this report.

Pipiringos was assisted in the field by E. Vernon Stephens in 1959, by Bob Helming in 1962, and by Kirby W. Bay in 1964. Assistance by others is acknowledged in Pipiringos (1968, p. D13) and in Pipiringos, Hail, and Izett (1969, p. N2). Thanks are due George H. Dixon, who helped with the preliminary compilation and drafting of all the figures in this report, and to M. A. Mullens who helped compile table 1 .

TABLE 1.-Location of control points and source of data used to construct restored sections and maps in this report

$[\mathrm{P}$, locality where chert pebbles and granules diagnostic of the $\mathrm{J}-2$ unconformity were collected; SCP, locality is a stratigraphic control point used to construct the maps and restored sections; $\mathrm{S}$, locality where chert pebble source beds were sampled; ONC, locality where pebbles were observed but not collected; LFNF, locality where the pebbles were looked for but not found; un, unsurveyed]

\begin{tabular}{|c|c|c|c|c|}
\hline $\begin{array}{l}\text { Locality } \\
\text { No }\end{array}$ & Locality name & $\begin{array}{c}\text { Sec., } \mathrm{T} ., \mathrm{R} \text {. } \\
\text { or } \\
\text { long } \mathrm{W} \text { and lat } \mathrm{N}\end{array}$ & Quadrangle & References and remarks \\
\hline
\end{tabular}

\begin{tabular}{|c|c|c|c|c|}
\hline \multicolumn{5}{|c|}{ Arizona } \\
\hline Al ............ & Cedar Mountain ................ & NE $1 / 4$ SW1/44, 41 N., 6 E. (un) ............ & Paria Plateau ...................... & $\begin{array}{l}\text { Fred Peterson (unpub. data, } \\
\text { 1971); P, SCP. }\end{array}$ \\
\hline A2 ........... & Ferry Swale ......................... & NE $1 / 4 \mathrm{SW} 1 / 421,41 \mathrm{~N} ., 8 \mathrm{E} \ldots \ldots \ldots \ldots \ldots \ldots . . . . .$. & Lees Ferry .......................... & $\begin{array}{l}\text { Phoenix (1963, p. 30), SCP. } \\
\text { Fred Peterson (unpub. data, } \\
\text { 1971), P, SCP. }\end{array}$ \\
\hline A3 .......... & 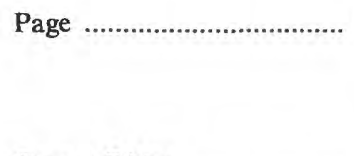 & SW1 $/ 4$ NW1 1419,41 N., 9 E. (un) .......... & Leche-e Rock ....................... & $\begin{array}{l}\text { Harshbarger and others (1957, } \\
\text { p. 14); SCP. Repenning and } \\
\text { others (1969, fig. 10, p. } \\
\text { B26); SCP. Fred Peterson } \\
\text { (unpub. data, 1971); P, SCP. }\end{array}$ \\
\hline A4 ........... & Garnet Ridge ........................ & 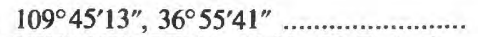 & Dinnehotso ................ & This report; $\mathbf{P}$. \\
\hline A5 ........... & West Red Mesa .................. & NW $1 / 4$ NE $1 / 4$ SE $1 / 44,41$ N., 28 E. (un) .. & Toh Atin Mesa ................... & Do. \\
\hline A6 ........... & East Red Mesa ................... & NW $1 / 4$ SE $1 / 4$ NE $1 / 46,41$ N., 29 E. (un) .. & ....... do ...................... & Strobell (1956); SCP. \\
\hline A7 ............ & Lefebre Canyon .................. & 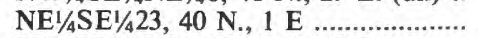 & 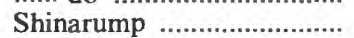 & This report; $\mathrm{S}$. \\
\hline A8 ............ & Marble Canyon ................... & $\mathrm{SE}^{1 / 4} \mathrm{SW} 1 / 4 \mathrm{SW} 1 / 432,40 \mathrm{~N} ., 7 \mathrm{E} \ldots \ldots \ldots .$. & Lees Ferry …........................ & Do. \\
\hline A9 $\ldots \ldots \ldots$ & Leche-e Rock ..................... & $111^{\circ} 16^{\prime} 37^{\prime \prime}, 36^{\circ} 52^{\prime} 17^{\prime \prime}$ & 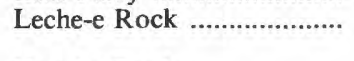 & $\begin{array}{l}\text { Fred Peterson (unpub. data, } \\
\text { 1971); SCP, ONC. }\end{array}$ \\
\hline A10 $\ldots \ldots . .$. & Tsai Skizzi Rock ................ & $11^{\circ} 06^{\prime} 43^{\prime \prime}, 36^{\circ} 49^{\prime} 20^{\prime \prime} \ldots \ldots$ & Navajo Creek ..................... & $\begin{array}{l}\text { Fred Peterson (unpub. data, } \\
\text { 1971); P. SCP. }\end{array}$ \\
\hline Al1 ........ & 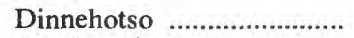 & $109^{\circ} 52^{\prime} 18^{\prime \prime}, 36^{\circ} 49^{\prime} 28^{\prime \prime}$ & 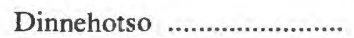 & This report; $\mathbf{S}$. \\
\hline A12 $\ldots \ldots . .$. & Antelope Pass ................... & $111^{\circ} 33^{\prime} 57^{\prime \prime}, 36^{\circ} 40^{\prime} 16^{\prime \prime} \ldots$ & Tanner Wash ..................... & $\begin{array}{l}\text { Fred Peterson (unpub. data, } \\
\text { 1971); P. }\end{array}$ \\
\hline $\mathrm{A} 13 \ldots \ldots . .$. & Klethla Valley ..................... & $110^{\circ} 29^{\prime} 10^{\prime \prime}, 36^{\circ} 34^{\prime} 02^{\prime \prime} \ldots$ & Long House Valley ... & $\begin{array}{l}\text { Fred Peterson (unpub. data, } \\
\text { 1971); P, SCP. }\end{array}$ \\
\hline A14 …...... & Carson Mesa .................... & $109^{\circ} 45^{\prime} 36^{\prime \prime}, 36^{\circ} 31^{\prime} 21^{\prime \prime} \ldots \ldots$. & 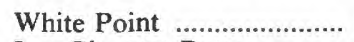 & This report; $P$. \\
\hline A15 & Toh Acon Mesa ................ & $109^{\circ} 22^{\prime} 30^{\prime \prime}, 36^{\circ} 39^{\prime} 12^{\prime \prime}$ & Los Gigantes Buttes ........... & This report; $P, S C P$. \\
\hline A16 .......... & Cove Mesa .......................... & $109^{\circ} 17^{\prime} 15^{\prime \prime}, 36^{\circ} 38^{\prime} 40^{\prime \prime} \ldots \ldots \ldots \ldots \ldots \ldots \ldots \ldots \ldots \ldots \ldots$ & ...... do .................................... & $\begin{array}{l}\text { Strobell }(1956) \text {; SCP. This } \\
\text { report, } \mathrm{P} \text {. }\end{array}$ \\
\hline
\end{tabular}


TABLE 1.--Location of control points and source of data used to construct restored sections and maps in this report-Continued

\begin{tabular}{lcccc}
\hline $\begin{array}{c}\text { Locality } \\
\text { No. }\end{array}$ & Locality name & $\begin{array}{c}\text { Sec., } \mathrm{T} ., \mathrm{R} . \\
\text { or } \\
\text { long } \mathrm{W} \text { and lat } \mathbf{N}\end{array}$ & Quadrangle & References and remarks \\
\hline
\end{tabular}

Arizona-Continued

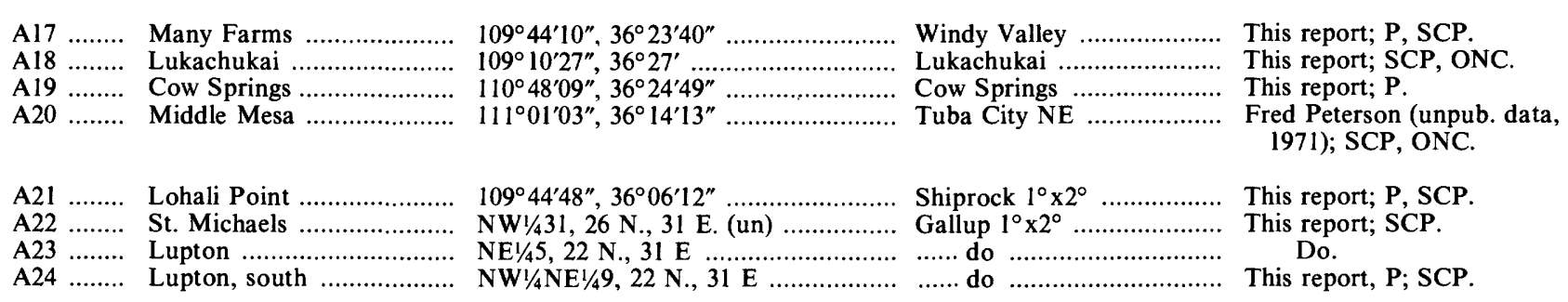

\section{Colorado}

\begin{tabular}{|c|c|}
\hline $\mathrm{C} 1 \ldots \ldots \ldots$ & Boundary Line \\
\hline $\mathrm{C} 2 \ldots \ldots \ldots$ & Bull Mountain .................... \\
\hline $\mathrm{C} 3 \ldots \ldots \ldots$ & Irish Lake .......................... \\
\hline $\mathrm{C} 4$............ & Boxelder, north . \\
\hline C5 .............. & Boxelder, south ................. \\
\hline C6 .. & 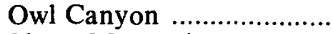 \\
\hline C7... & Sheep Mountain ............... \\
\hline $\mathrm{C} 8 \ldots \ldots \ldots$ & Moon Hill ....................... \\
\hline $\mathrm{C} 9 . . . \ldots \ldots$ & Ditch Creek \\
\hline $\mathrm{C} 10 \ldots \ldots .$. & Bellvue ....... \\
\hline
\end{tabular}

C11 ....... Little Snake River ........... S1/2SW1/429, 7 N., 98 W

C12 $12 . . .$. Cross Mount

C13 .......... Cameron Pass

C14

Cl6 ........ Deerlodge Park

C17 ......... Calico Draw

C18 ......... Miller Creek

C19 ........ Frantz Creek

C20 ........ Little Thompson

C21 ........ Uranium Peak

C22 ......... Fawn Creek

C23 ......... Muddy Slide

C24 ......... Kremmling

C25 Hot Sulphur Springs

C26 ........ Tabernash

C27 ........ Four Mile Canyon ............

C28 .......... LO 7 Hill

C29 ....... Burns

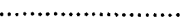

C31 ........ Radium, southwest

C32 ....... Ralston Reservoir ............

C33 ........ Middle Rifle Creek ............

C34 ........ Main Elk Creek ................

C35 ........ South Canyon Creek ........

C36 ....... Aspen

C37 ....... Red Hill Gap
NE $1 / 4 \mathrm{SE}^{1 / 4} \mathrm{NW} 1 / 430,12 \mathrm{~N} ., 69 \mathrm{~W}$ NE $1 / 4$ NW $1 / 4$ NW $1 / 416,11$ N., 76 W SW $1 / 4$ SW $1 / 4$ SW $1 / 434,11$ N., 101 W C E $1 / 2 E^{1 / 2} 30,11$ N., 69 W SE $1 / 4$ SW $1 / 4$ NW $1 / 48,10$ N., 69 W

SW $1 / 4$ NE $1 / 4$ NW $1 / 45,9$ N., 69 W SE $1 / 4$ SE $1 / 4$ NEL $/ 44,9$ N., 81 W SW $1 / 4$ NW $^{1} / 4$ NW $^{1} / 416,8$ N., 85 W SW $1 / 4$ NW $1 / 4$ NE $1 / 425,8$ N., 85 W SE $1 / 4$ NW $1 / 4$ NE $1 / 431,8$ N., 69 W NW $1 / 4$ NW $1 / 4$ NW $1 / 44,6$ N., 98 W ...... C E $1 / 2$ W $1 / 2$ NE $1 / 411,6$ N., 76 W. (un). SE $1 / 4$ SW $1 / 4$ SW $1 / 48,5$ N., 69 W C W $1 / 2$ NE $1 / 4$ NW $1 / 424,5$ N., 70 W ......

NE $1 / 4 N^{1 / 4}$ NW $^{1} / 428,6$ N., 99 W SE $1 / 4$ SE $1 / 4$ SW $1 / 44,5$ N., 99 W ......... SE $1 / 4$ SW $1 / 427,4$ N., 101 W SE $1 / 4 N^{\prime} 1 / 4$ SE $1 / 432,4$ N., 82 W

C NEL $/ 4 N^{2} 1 / 44,3$ N., 70 W

SE $1 / 4$ NW $1 / 4$ NE $1 / 428,2$ N., 92 W NE $1 / 4$ NE $1 / 418,1$ N., $90 \mathrm{~W}$

NE $1 / 414,2$ N., 84 W

NE $1 / 4$ NE $1 / 4$ NW $1 / 421,1$ N., 81 W

NE $1 / 4$ NW $1 / 4$ NE $1 / 410,1$ N., 78 W

SW $1 / 4$ SE $1 / 4$ NE $1 / 432,1$ N., 76 W

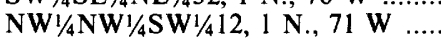

C W $1 / 230,1$ S. 93 W

C $28,2 \mathrm{~S}, 85 \mathrm{~W}$

SW $1 / 433,1$ S., 84 W

SW $1 / 4$ NW $1 / 427,1$ S., 82 W

NE $1 / 4$ NW $1 / 4$ SW $1 / 45,3$ S., 70 W SW $1 / 4$ SW $1 / 4$ NE $1 / 436,4$ S., 93 W SE $1 / 410,5$ S., $91 \mathrm{~W}$

C W $1 / 2$ SE $1 / 42,6$ S., $90 \mathrm{~W}$

SE $1 / 4$ NW $1 / 4 N E 1 / 420,10$ S., 85 W. (un)

NE $1 / 414,9$ S., 77 W
Table Mountain Crazy Mountain

Irish Canyon

Table Mountain ...............

Livermore

...... do

Lake John

Clark

Floyd Peak .........................

Horsetooth Reservoir ........

Lone Mountain

...... do

Clark Peak

Masonville

...... do .

Indian Water Canyon

...... do

Skull Creek

Lake Agnes

Carter Lake Reservoir .....

Sawmill Mountain

Fawn Creek

Craig ........

Kremmling

ot Sulphur Springs

Granby

Boulder

LO 7 Hill

Leadville $1^{\circ} \times 2^{\circ}$

.... do

Ralston Buttes

Horse Mountain

Leadville $1^{\circ} \times 2^{\circ}$

Storm King Mountain ....

Highland Peak

Como
This report; SCP.

Pipiringos (1957, p. 61); ONC.

This report; $P, S C P$.

Do.

Do.

Do.

This report; SCP, ONC.

Do.

This report; $P, S C P$.

Do.

Do.

This report; ONC.

This report; $P$.

This report; $\mathbf{P}, \mathbf{S C P}$.

Do.

This report; $\mathbf{P}$.

This report; $P$, SCP. Do.

Pipiringos and others (1969, p. N31, sec. J); P, SCP.

This report; $P, \mathbf{S C P}$.

This report; ONC.

This report; SCP, S.

This report; $P, S C P$. Do.

Izett (1968, p. 14, fig. 6 and p. 17, beds 1 and 2).

This report; P,SCP.

This report; $\mathbf{P}, \mathbf{S C P}$

Do.

This report; $\mathbf{S}$.

This report; $P$

Pipiringos and others (1969, p. N16, sec. A); P.

Pipiringos and others (1969, p. N18, sec. B); P. SCP.

This report; $\mathbf{P}, \mathbf{S C P}$.

This report; $P$

Fischer (1960, p. 10, fig. 2, sec. 14); SCP.

This report; SCP, ONC.

B.H. Bryant (unpub. data, 1965); P.

Stark and others (1949, p. 47); SCP, ONC. 
TABLE 1.-Location of control points and source of data used to construct restored sections and maps in this report-Continued

\begin{tabular}{|c|c|c|c|c|}
\hline $\begin{array}{l}\text { Locality } \\
\text { No. }\end{array}$ & Locality name & $\begin{array}{c}\text { Sec., T., } \mathrm{R} \text {. } \\
\text { or } \\
\text { long } \mathrm{W} \text { and lat } \mathrm{N}\end{array}$ & Quadrangle & References and remarks \\
\hline \multicolumn{5}{|c|}{ Colorado-Continued } \\
\hline C38 ......... & 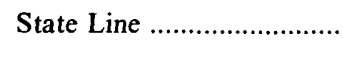 & 6, 11 S., $104 \mathrm{~W}$ & Bitter Creek Well ............... & $\begin{array}{l}\text { Wright and others (1962, } \\
\text { p. 2061, fig. 2, sec. 8); SCP. }\end{array}$ \\
\hline C39 ......... & $\begin{array}{l}\text { Colorado National } \\
\text { Monument. }\end{array}$ & $\mathrm{SE}^{1 / 4} \mathrm{SE}^{1 / 4} \mathrm{NW} 1 / 48,12 \mathrm{~S} ., 101 \mathrm{~W}$. (un) & $\begin{array}{l}\text { Colorado National .............. } \\
\text { Monument }\end{array}$ & This report; SCP, ONC. \\
\hline $\mathrm{C} 40 \ldots \ldots .$. & 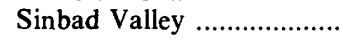 & NW $1 / 4 N W 1 / 431,50$ N., 19 W. (un) $\ldots .$. & Polar Mesa ........................... & Cater (1970, p. 29); SCP, ONC. \\
\hline $\mathrm{C} 41 \ldots \ldots . .$. & Atkinson Mesa ................... & $\mathrm{SW}^{1} / 4 \mathrm{NE}^{1} / 4 \mathrm{NE}^{1} / 423,48 \mathrm{~N} ., 18 \mathrm{~W} \ldots \ldots$. & Paradox & $\begin{array}{l}\text { Fred Peterson (unpub. data, } \\
\text { 1971); SCP, ONC. }\end{array}$ \\
\hline C42 ......... & Uravan …........................... & 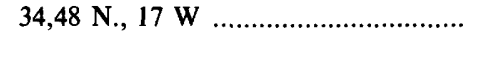 & 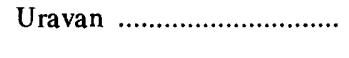 & $\begin{array}{l}\text { Cater (1970, p. 12, fig. } 4 \text {; } \\
\text { p. } 28, \text { fig. 8); SCP. }\end{array}$ \\
\hline $\mathrm{C} 43 \ldots \ldots \ldots$ & La Sal Creek ........................ & $\mathrm{SE}^{1 / 4} \mathrm{SW} 1 / 4 \mathrm{NW} 1 / 417,47 \mathrm{~N} ., 19 \mathrm{~W} \ldots . .$. & Paradox & $\begin{array}{l}\text { Fred Peterson (unpub. data, } \\
\text { 1971); SCP, ONC. }\end{array}$ \\
\hline C44 .......... & Slick Rock . ...................... & $\mathrm{SW}^{1 / 4} \mathrm{SE}^{1 / 4} \mathrm{NW} \mathrm{W}^{1} / 425,44 \mathrm{~N} ., 19 \mathrm{~W} \ldots . .$. & Horse Range Mesa ............ & $\begin{array}{l}\text { Cater (1970, p. 29), P, SCP. } \\
\text { This report; P. }\end{array}$ \\
\hline $\mathrm{C} 45 \ldots \ldots .$. & McElmo Dome .................... & 26,36 N., $18 \mathrm{~W}$ & 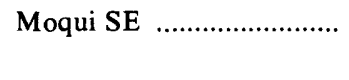 & $\begin{array}{l}\text { Ekren and Houser (1965, p. 7); } \\
\text { SCP. }\end{array}$ \\
\hline $\begin{array}{l}\mathrm{C} 46 \\
\mathrm{C} 47\end{array}$ & 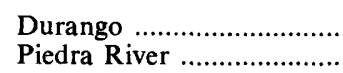 & $\begin{array}{l}\text { NW1/4NW1/4NW1/44, } 35 \text { N., } 9 \text { W ...... } \\
\text { SE1/4SW1/4NW1/45, } 34 \text { N., } 4 \text { W ......... }\end{array}$ & $\begin{array}{l}\text { Durango, East } \\
\text { Chimney Rock }\end{array}$ & $\begin{array}{l}\text { This report; P, SCP. } \\
\text { Do. }\end{array}$ \\
\hline C48 $\ldots \ldots \ldots$ & Pass Creek .............................. & $\mathrm{SW} 1 / 4 \mathrm{NE}^{1} / 4 \mathrm{NE} 1 / 418,27 \mathrm{~S} ., 70 \mathrm{~W} \ldots \ldots$. & Red Wing ............................... & $\begin{array}{l}\text { Johnson and Baltz (1960, } \\
\text { p. 1898, fig. 2, sec. 2); } \\
\text { SCP, ONC. This report; P. }\end{array}$ \\
\hline $\mathrm{C} 49 \ldots \ldots .$. & 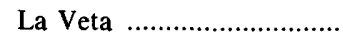 & SW $1 / 4$ SE $^{1} / 4 \mathrm{SW}^{1} / 426,30 \mathrm{~S} ., 69 \mathrm{~W} \ldots \ldots \ldots$ & 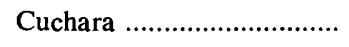 & This report, $\mathrm{P}, \mathrm{SCP}$. \\
\hline \multicolumn{5}{|c|}{ Idaho } \\
\hline I1 ................ & Garns Mountain ................ & 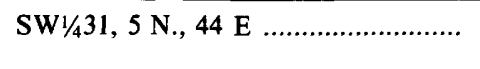 & Garns Mountain .................. & $\begin{array}{l}\text { Staatz and Albee (1966, } \\
\text { p. 49); SCP. }\end{array}$ \\
\hline & Willow Creek ........................ & $\mathrm{NE}^{1 / 4} 19,1$ N., 40 E & Ammon & Imlay $(1967$, p. 22); SCP, ONC. \\
\hline $13 \ldots \ldots \ldots . .$. & Stump Creek ...................... & NW $1 / 416 ;$ SE $1 / 428,6$ S., 45 E. (un) .... & Freedom ............................ & $\begin{array}{l}\text { R. W. Imlay and G. N. Pipiringos } \\
\text { (unpub. data, 1975); SCP. } \\
\text { Imlay (1967, p. 22, fig. 4, } \\
\text { sec. 16), SCP, ONC. }\end{array}$ \\
\hline I4 & 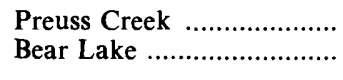 & $\begin{array}{l}\text { NE } 1 / 415,11 \text { S., } 45 \text { E. (un) } \\
\mathrm{NE}^{1 / 430} 30,15 \text { S., } 45 \text { E }\end{array}$ & $\begin{array}{l}\text { Meade Peak .......................... } \\
\text { Pegram Creek }\end{array}$ & $\begin{array}{l}\text { Imlay (1967, p. 22); ONC. } \\
\text { Do. }\end{array}$ \\
\hline \multicolumn{5}{|c|}{ Montana } \\
\hline & $\begin{array}{l}\text { R.G. Parrent } 1 \\
\text { McAlpine. }\end{array}$ & NW $1 / 4 \mathrm{SE}^{1} / 425,37$ N., $3 \mathrm{~W}$ & 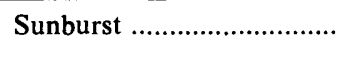 & $\begin{array}{l}\text { Cobban }(1945, \text { p. 1302-1303); } \\
\text { ONC. }\end{array}$ \\
\hline & $\begin{array}{l}\text { Carter Oil Co. } 25 \\
\text { Tribal. }\end{array}$ & SE $1 / 4 N W 1 / 427,36$ N., $6 \mathrm{~W} \ldots \ldots \ldots \ldots \ldots$ & Dead Indian Spring & $\begin{array}{l}\text { Cobban }(1945, \text { p. 1301-1302); } \\
\text { ONC. }\end{array}$ \\
\hline M3 .......... & $\begin{array}{l}\text { Hannah-Porter Co. } 1 \\
\text { Marceau. }\end{array}$ & 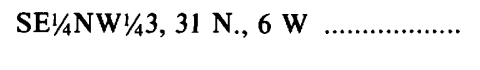 & Lake Frances ........................ & $\begin{array}{l}\text { Cobban (1945, p. 1300-1301; } \\
\text { SCP, ONC. }\end{array}$ \\
\hline M4 …...... & 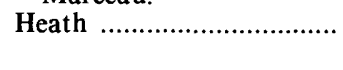 & 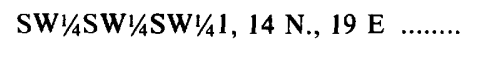 & 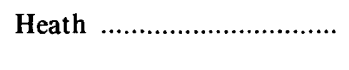 & $\begin{array}{l}\text { Imlay and others (1948, sec. } \\
\text { 12); SCP. This report; P. }\end{array}$ \\
\hline M5 .......... & Stone House ......................... & 32 and 33,11 N., 21 E & Roundup $1^{\circ} \times 2^{\circ}$ & $\begin{array}{l}\text { Imlay and others (1948, sec. } \\
\text { 13); SCP. }\end{array}$ \\
\hline M6 ............ & $\begin{array}{l}\text { Mobil Oil Corp. T-86-9 } \\
\text { Northern Pacific. }\end{array}$ & $\mathrm{SE}^{1} / 4 \mathrm{NE}^{1} / 4 \mathrm{SE}^{1} / 49,8 \mathrm{~N} ., 20 \mathrm{E} . . . \ldots \ldots \ldots . . .$. & 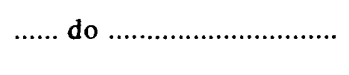 & This report; SCP. \\
\hline M7 ............. & $\begin{array}{l}\text { Texas Pacific Coal and } \\
\text { Oil Co. } 1 \text { Northern } \\
\text { Pacific. }\end{array}$ & $\mathrm{SW}^{1} / 4 \mathrm{SW} 1 / 4 \mathrm{SW}^{1} / 421,8 \mathrm{~N} ., 21 \mathrm{E} \ldots \ldots .$. & ...... do ................................ & $\begin{array}{l}\text { Imlay and others }(1948, \text { sec. } \\
\text { 14); SCP. }\end{array}$ \\
\hline M8 ........... & $\begin{array}{l}\text { DeKalb Oil Co. 8-7 } \\
\text { Northern Pacific. }\end{array}$ & $\mathrm{SW}^{1} 1 / 4 \mathrm{SW}^{1} / 4 \mathrm{SW}^{1} / 47,5 \mathrm{~N} ., 19 \mathrm{E} \ldots \ldots \ldots$. & ...... do ................................ & This report; SCP. \\
\hline M9 .......... & $\begin{array}{l}\text { Rocky Mountain } \\
\text { Exploration 13-36 } \\
\text { State. }\end{array}$ & $\mathrm{SW}^{1} / 4 \mathrm{SW}^{1} / 436,4 \mathrm{~N} ., 20 \mathrm{E}$ & ....... do .............................. & Do. \\
\hline $\mathrm{M} 10 \ldots \ldots$. & $\begin{array}{l}\text { U.S. Smelting, Mining, } \\
\text { and Refining, Voss } \\
\text { Oil Co. } 1 \text { Gee. }\end{array}$ & NW1/4NW1/429, 2 N., 20 E ..................... & 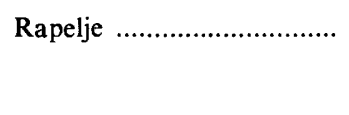 & Do. \\
\hline M11 ….... & $\begin{array}{l}\text { U.S. Smelting, Mining, } \\
\text { and Refining, Voss } \\
\text { Oil Co. } 1 \text { Pelton. }\end{array}$ & 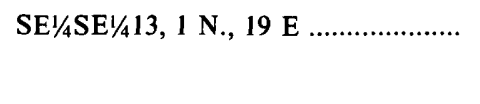 & Lindemulder Hill .................. & Do. \\
\hline
\end{tabular}


TABLE 1.-Location of control points and source of data used to construct restored sections and maps in this report-Continued

\begin{tabular}{cccc}
\hline $\begin{array}{c}\text { Locality } \\
\text { No. }\end{array}$ & Locality name & $\begin{array}{c}\text { Sec., T., } \mathrm{R} . \\
\text { or } \\
\text { long W and lat } \mathrm{N}\end{array}$ & Quadrangle \\
\hline
\end{tabular}

Montana-Continued

\begin{tabular}{|c|c|c|c|c|}
\hline M12 $\ldots \ldots . .$. & Benbow Mill Road ............ & 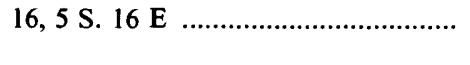 & Mount Wood .................... & $\begin{array}{l}\text { Imlay and others (1948, sec. } \\
26) ; . \text { SCP. }\end{array}$ \\
\hline M13 & Five Mile Creek ............... & $\begin{array}{l}36,5 \mathrm{~S} ., 24 \text { E. and } 2,6 \mathrm{~S} . \\
24 \text { E. }\end{array}$ & Section House Draw ........ & $\begin{array}{l}\text { Imlay and others }(1948, \text { sec. } \\
\text { 27); SCP. }\end{array}$ \\
\hline $\begin{array}{l}\text { M14 } \\
\text { M15 }\end{array}$ & $\begin{array}{l}\text { Ohio Oil Co. } 24 \text { Chapman. } \\
\text { Red Dome ........................ }\end{array}$ & $\begin{array}{l}\text { C SE1/4SW1/42, } 7 \text { S., } 21 \text { E ................. } \\
\text { NE1/4NE1/419, } 7 \text { S., } 24 \text { E ................. }\end{array}$ & $\begin{array}{l}\text { Belfry } \\
\text { Wade } \ldots \ldots \ldots \ldots \ldots \ldots \ldots \ldots \ldots \ldots \ldots \ldots \ldots \ldots \ldots\end{array}$ & $\begin{array}{l}\text { This report; SCP. } \\
\text { Imlay and others (1948, sec. } \\
\text { 16); SCP. This report, P. }\end{array}$ \\
\hline M16 $\ldots \ldots . .$. & Gardiner .......................... & 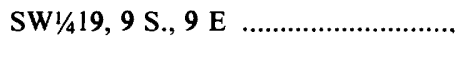 & 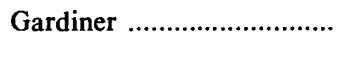 & $\begin{array}{l}\text { Fraser and others }(1969, \text { p.21 } \\
\text { and pl. 1); SCP, ONC. }\end{array}$ \\
\hline M17 $\ldots \ldots . .$. & $\begin{array}{l}\text { Carter Oil Co. } 1 \\
\text { Wheatly-Government. }\end{array}$ & 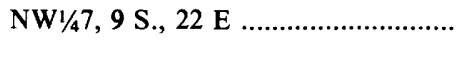 & Hollenbeck Draw .............. & This report; SCP. \\
\hline M18 $\ldots . . .$. & Gypsum Creek ................. & 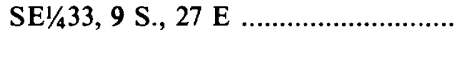 & Red Pryor Mountain ........ & $\begin{array}{l}\text { Imlay }(1956, \text { p. } 568, \text { sec. } 10) \\
\text { ONC. This report; P. }\end{array}$ \\
\hline M19 $\ldots \ldots . .$. & Lodge Grass Creek ............ & NW1/4SW1/4SW1/48, 9 S., 33 E .......... & Willow Creek Dam SW ... & $\begin{array}{l}\text { Imlay }(1956, \text { p. } 566, \text { sec. } 6) \\
\text { ONC. This report; P. }\end{array}$ \\
\hline
\end{tabular}

\begin{tabular}{|c|c|c|c|c|}
\hline \multicolumn{5}{|c|}{ New Mexico } \\
\hline & Ricardo Creek ....................... & $105^{\circ} 09^{\prime} 30^{\prime \prime}, 36^{\circ} 59^{\prime} 09^{\prime \prime}$. & 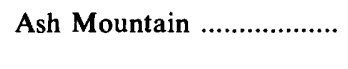 & $\begin{array}{l}\text { C. L. Pillmore (unpub. data, } \\
\text { 1967); P. SCP. }\end{array}$ \\
\hline NM2 ....... & (n) & $\mathrm{SW} 1 / 4 \mathrm{NE} 1 / 4 \mathrm{SE} 1 / 432,32 \mathrm{~N} ., 33 \mathrm{E} \ldots \ldots .$. & Travesser Park .................. & $\begin{array}{l}\text { W. I. Finch (unpub. data, } \\
\text { 1970); P. }\end{array}$ \\
\hline $\begin{array}{l}\text { NM3 } \\
\text { NM4 }\end{array}$ & Battleship Mountain ......... & $\begin{array}{l}\mathrm{SE}^{1} / 4 \mathrm{NW}^{1} / \mathrm{S} \mathrm{SE}^{1} / 428,32 \mathrm{~N} ., 35 \mathrm{E} \ldots \ldots . . \\
18^{\circ} 53^{\prime} 34^{\prime \prime}, 36^{\circ} 14^{\prime} 48^{\prime \prime}\end{array}$ & Wedding Cake Butte ......... & $\begin{array}{l}\text { Do. } \\
\text { This report: SCP ONC }\end{array}$ \\
\hline NM5 ........ & 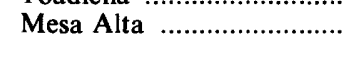 & $\mathrm{NE}^{1 / 4} \mathrm{NW} 1 / 4 \mathrm{NW} 1 / 4,23,23 \mathrm{~N} ., 1 \mathrm{E}$ & 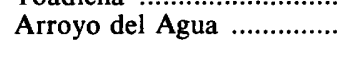 & $\begin{array}{l}\text { M. W. Green (unpub. data, } \\
\text { 1970); P. }\end{array}$ \\
\hline NM6 ...... & 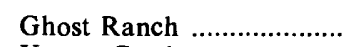 & 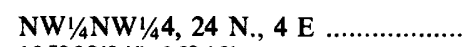 & Echo Amphitheater ........... & This report; $P$. \\
\hline NM7 ...... & Urraca Creek .................... & 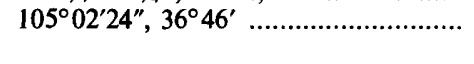 & Tooth of Time ..................... & $\begin{array}{l}\text { M. W. Green (unpub. data, } \\
\text { 1970); LFNF. }\end{array}$ \\
\hline NM8 ...... & Gonzales Hill ....................... & $\mathrm{NE} 1 / 42,20$ N., $30 \mathrm{E}$ & Tucumcari $1^{\circ} \times 2^{\circ}$ & $\begin{array}{l}\text { W. I. Finch (unpub. data, } \\
\text { 1970); ONC. }\end{array}$ \\
\hline $\begin{array}{l}\text { NM9 } \\
\text { NM10.... }\end{array}$ & $\begin{array}{l}\text { Todilto Park . } \\
\text { Navajo }\end{array}$ & $\begin{array}{l}108^{\circ} 58^{\prime} 15^{\prime \prime}, 35^{\circ} 57^{\prime} 15^{\prime \prime} \\
109^{\circ} 01^{\prime} 45^{\prime \prime}, 35^{\circ} 49^{\prime} 50^{\prime \prime}\end{array}$ & 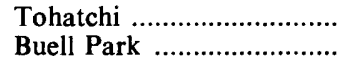 & $\begin{array}{l}\text { This report; } S C P, L F N F \text {. } \\
\text { This report; } P, S C P \text {. }\end{array}$ \\
\hline NM11 $\ldots$. & Cachana Spring ...................... & NW $1 / 4 \mathrm{SW}_{1} 1 / 4 \mathrm{SE} 1 / 436,17 \mathrm{~N} ., 1 \mathrm{~W}$. (un) & Holy Ghost Spring ............. & $\begin{array}{l}\text { M. W. Green (unpub. data, } \\
\text { 1972); P. }\end{array}$ \\
\hline $\begin{array}{l}\mathrm{NM} 12 \\
\mathrm{NM} 13 \ldots\end{array}$ & 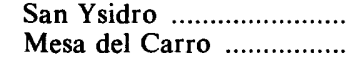 & $\begin{array}{l}\text { NE } 1 / 4 \mathrm{SW}^{1} / 4 \mathrm{NE}^{1} / 425,16 \mathrm{~N} ., 1 \mathrm{~W} \text {. (un) } \\
105^{\circ} 02^{\prime} 27^{\prime \prime}, 35^{\circ} 22^{\prime} 30^{\prime \prime}\end{array}$ & $\begin{array}{l}\text { Ojito Spring } \\
\text { Apache Spring }\end{array}$ & $\begin{array}{l}\text { Do. } \\
\text { Do. }\end{array}$ \\
\hline NM $14 \ldots$. & Montoya & $\mathrm{SE}^{1 / 4} \mathrm{SE}^{1} / 4 \mathrm{NE} 1 / 410,17$ N., 26 E. (un) & Montoya Point .................... & $\begin{array}{l}\text { W. I. Finch (unpub. data, } \\
\text { 1970); LFNF. }\end{array}$ \\
\hline NM15 .... & 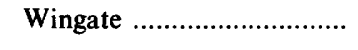 & 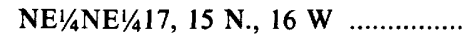 & 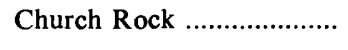 & This report; $P, S C P$. \\
\hline NM16 .... & 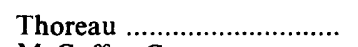 & $\mathrm{NE}^{1 / 4} \mathrm{NE}^{1 / 4} 19,14 \mathrm{~N} ., 12 \mathrm{~W}$. & Thoreau ................................. & This report; $\mathbf{P}$. \\
\hline NM17 .... & 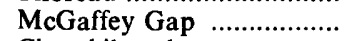 & $\mathrm{NE}^{1 / 4} \mathrm{NW} 1 / 413,13 \mathrm{~N} ., 17 \mathrm{~W} \ldots \ldots \ldots \ldots . . . . .$. & Upper Nutria .......................... & This report; P, SCP. \\
\hline NM18 .... & Cheechilgeetho ...................... & $\mathrm{SW}^{1} / 4 \mathrm{NE}^{1 / 4 \mathrm{SE}^{1} / 413,12 \mathrm{~N} ., 20 \mathrm{~W} \ldots \ldots . .}$ & Jones Ranch School .......... & Do. \\
\hline NM19 .... & Nutria & 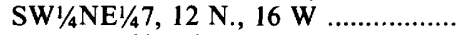 & Upper Nutria .......................... & This report; ONC. \\
\hline NM20 .... & 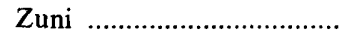 & SW $1 / 48,9$ N., $19 \mathrm{~W}$ & Gallup $1^{\circ} \times 2^{\circ} \ldots \ldots \ldots \ldots \ldots \ldots \ldots$ & This report; SCP. \\
\hline NM21 .... & Cheama Canyon ................... & 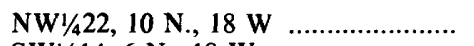 & ....... do ................ & Do. \\
\hline NM22 .... & Atarque ................................ & 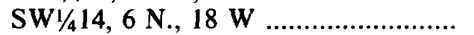 & St. Johns $1^{\circ} \times 2^{\circ} \ldots \ldots \ldots \ldots \ldots \ldots$ & This report; $P, S C P$. \\
\hline NM23 .... & Acoma $\ldots \ldots \ldots \ldots \ldots \ldots \ldots \ldots \ldots \ldots \ldots$ & SW $1 / 4$ NE $^{1 / 4}$ NW $1 / 42,7$ N., 7 W .......... & East Mesa .............................. & $\begin{array}{l}\text { M. W. Green (unpub. data, } \\
\text { 1970); P. }\end{array}$ \\
\hline NM24 .... & Petoch Butte ......................... & 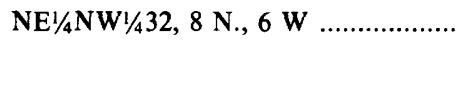 & 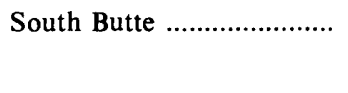 & $\begin{array}{l}\text { Moench and Schlee (1967, } \\
\text { p. 7); ONC. This report; } \\
\text { SCP. }\end{array}$ \\
\hline NM25 .... & Luciano Mesa ................... & $\mathrm{NE}^{1 / 4} \mathrm{NE} 1 / 4 \mathrm{SW} 1 / 48,8 \mathrm{~N} ., 27 \mathrm{E} \ldots \ldots \ldots . .$. & Ima & $\begin{array}{l}\text { M. W. Green (unpub. data, } \\
\text { 1970); P. SCP. }\end{array}$ \\
\hline
\end{tabular}

\section{Oklahoma}

\begin{tabular}{|c|c|c|c|c|}
\hline OK1 $1 \ldots \ldots$ & Carrizozo Creek .................. & NE1/4NW1/4SW1/418, 5 N., 1 E .......... & 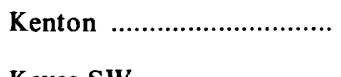 & $\begin{array}{l}\text { W. I. Finch (unpub. data, } \\
\text { 1971); ONC. }\end{array}$ \\
\hline OK2 . & Picket House Draw & SW & 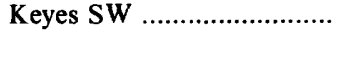 & $\begin{array}{l}\text { W. I. Finch (unpub. data, } \\
\text { 1971); ONC, SCP. }\end{array}$ \\
\hline
\end{tabular}


TABLE 1.-Location of control points and source of data used to construct restored sections and maps in this report-Continued

\begin{tabular}{|c|c|c|c|c|}
\hline $\begin{array}{l}\text { Locality } \\
\text { No. }\end{array}$ & Locality name & $\begin{array}{c}\text { Sec., } T ., R \text {. } \\
\text { or } \\
\text { long } W \text { and lat } N\end{array}$ & Quadrangle & References and remarks \\
\hline \multicolumn{5}{|c|}{ South Dakota } \\
\hline SDl $\ldots . . . .$. & Redwater Creek .................. & NW1/4SW1/4NE1/411, 7 N., I E .......... & Belle Fourche ....................... & $\begin{array}{l}\text { Imlay (1947, p. 267); ONC. } \\
\text { Mapel and Bergendahl (1956, } \\
\text { p. 87); ONC. This report; P. }\end{array}$ \\
\hline SD2 $\ldots \ldots .$. & Rapid City . & SE1/434, 1 N., 7 E & Rapid City West ............... & $\begin{array}{l}\text { Imlay }(1947, \text { p. 267-268); } \\
\text { SCP. }\end{array}$ \\
\hline SD3 …..... & Dewey & $\mathrm{SE}^{1} / 4 \mathrm{NW}^{1 / 4} \mathrm{NE} 1 / 410,6 \mathrm{~S} ., 1 \mathrm{E} . . . \ldots \ldots \ldots . . . .$. & Jewell Cave SW ................ & $\begin{array}{l}\text { Imlay }(1947, \text { p. } 252) ; \text { SCP, } \\
\text { ONC. Braddock (1963, } \\
\text { p. 232); SCP, ONC. }\end{array}$ \\
\hline & Minnekahta ........................... & & Minnekahta ........................... & $\begin{array}{l}\text { Imlay (1947, p. } 235 \text { and } 273) \\
\text { ONC, SCP. This report; P. }\end{array}$ \\
\hline SD5 …..... & 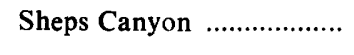 & NW1/4NE1/415, 8 S., 5 E ....................... & Cascade Springs ....................... & Post $(1967$, p. 452$) ;$ ONC. \\
\hline \multicolumn{5}{|c|}{ Utah } \\
\hline $\mathrm{U} 1$ & Devils Slide ......................... & 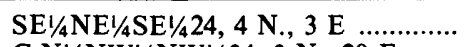 & Devils Slide ........................... & This report; SCP, S. \\
\hline $\mathrm{U} 2 \ldots \ldots \ldots$ & Red Bench ............................ & C N1/2NW1/4NW1/434, 3 N., 20 E ...... & Manila .................................. & This report; P, SCP. \\
\hline U3 …......... & Sheep Creek Gap .............. & NW1/4NW1/4NW1/47, 2 N., 20 E ......... & 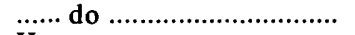 & Do. \\
\hline U4 ............ & 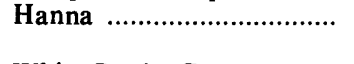 & $\mathrm{NE}^{1 / 4} \mathrm{SE} 1 / 4 \mathrm{SE} 1 / 44,1 \mathrm{~S} ., 8 \mathrm{~W} \ldots \ldots \ldots \ldots . . .$. & 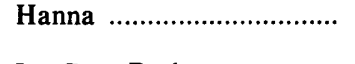 & $\begin{array}{l}\text { Imlay (1967); SCP. This } \\
\text { report; ONC. }\end{array}$ \\
\hline U5 ............. & White Rocks Canyon ....... & SW $1 / 418$ and NW1/419, 2 N., 1 E ....... & Ice Cave Peak ...................... & $\begin{array}{l}\text { Kinney }(1955, \text { pl. } 2) ; \text { SCP. } \\
\text { Imlay (1967, p. 13): SCP. } \\
\text { This report; LFNF. }\end{array}$ \\
\hline U6 ............... & Steinaker Draw ..................... & NW1/4NW1/426, 3 S., 21 E .................. & Steinaker Reservoir ........... & $\begin{array}{l}\text { Kinney }(1955) \text {; ONC. This } \\
\text { report; P, SCP. }\end{array}$ \\
\hline $\mathrm{U} 7 \ldots \ldots \ldots$ & Brush Creek ........................ & SE1/4NW1/4NW1/42, 3S., 22 E ............ & Donkey Flat ......................... & This report; $P$ \\
\hline U8 .............. & 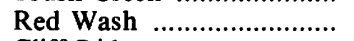 & $\mathrm{SE} 1 / 4 \mathrm{NE} 1 / 4 \mathrm{SW} 1 / 425,4 \mathrm{~S} ., 23 \mathrm{E}$ & Dinosaur Quarry ................... & This report; ONC. \\
\hline U9 ............... & 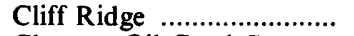 & SW $1 / 4 N^{N} 1 / 4 S^{\prime} 1 / 43,6$ S., 24 E ........... & Cliff Ridge ............................ & This report; SCP, ONC. \\
\hline $\mathrm{U} 10 \ldots \ldots . .$. & $\begin{array}{l}\text { Chevron Oil Co. } 1 \text { Stone } \\
\text { Cabin. }\end{array}$ & NW $1 / 4 \mathrm{NE}^{1} / 429,12 \mathrm{~S} ., 15 \mathrm{E}$ & Sunnyside ............................. & This report; SCP. \\
\hline U11 .......... & $\begin{array}{l}\text { Pan American Corp. } 1 \\
\text { USA Farnham. }\end{array}$ & $\mathrm{SW} 1 / 4 \mathrm{SW}^{1} / 47,15 \mathrm{~S} ., 12 \mathrm{E} \ldots \ldots \ldots \ldots \ldots \ldots . . . . . . .$. & Wellington ............................ & Do. \\
\hline $\mathrm{U} 12 \ldots \ldots .$. & Buckhorn Wash ............... & NW1/4SE1/4SW1/418, 19 S., 11 E ........ & Bob Hill Knoll ...................... & This report; P, SCP. \\
\hline U13 $\ldots \ldots .$. & Smith Cabin .......................... & $\mathrm{C} \mathrm{N}^{1} / 2 \mathrm{~N}^{1} / 25,21$ S., $14 \mathrm{E} . \ldots \ldots \ldots \ldots \ldots \ldots$ & Beckwith Peak SW ........... & Do. \\
\hline U14 $\ldots \ldots .$. & Justensen Flats ..................... & 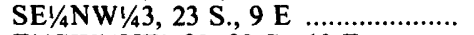 & San Rafael Knob ................. & Do. \\
\hline U15 ......... & Straight Wash .................... & $\mathrm{E}^{1} / 2 \mathrm{SW} 1 / 4 \mathrm{NE}^{1 / 428,} 23 \mathrm{~S} ., 13 \mathrm{E} \ldots \ldots \ldots \ldots$. & Tidwell Bottoms ................. & Do. \\
\hline U16 ........... & Dewey Bridge ....................... & 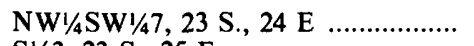 & Cisco & This report; ONC. \\
\hline $\mathrm{U} 17 \ldots \ldots .$. & Scharf Mesa .......................... & $\mathrm{S} 1 / 23,23 \mathrm{~S} ., 25 \mathrm{E}$ & Coates Creek ........................ & $\begin{array}{l}\text { Dane (1935), and Williams } \\
\text { (1964); SCP. }\end{array}$ \\
\hline $\mathrm{U} 18 \ldots \ldots . .$. & North Temple Wash .......... & $\mathrm{SE}^{1} / 4 \mathrm{SW} 1 / 4 \mathrm{SE} 1 / 41,25 \mathrm{~S} ., 11$ E. (un) .... & Temple Mountain ............. & This report; $\mathbf{P}$ : \\
\hline U19 $\ldots \ldots \ldots$ & Sevenmile Canyon .............. & NW1/4SW1/412, 25 S., 19 E. (un) ....... & The Knoll ................................. & $\begin{array}{l}\text { Wright and Dickey (1957); ONC, } \\
\text { S. This report; P, S. }\end{array}$ \\
\hline $\mathrm{U} 20 \ldots \ldots .$. & $\begin{array}{l}\text { Arches National } \\
\text { Monument. }\end{array}$ & 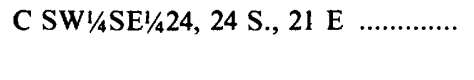 & 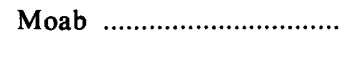 & This report; $\mathbf{P}, \mathbf{S}$. \\
\hline $\mathrm{U} 21 \ldots \ldots . .$. & Black Ridge .............................. & $\mathrm{NE}^{1 / 419}$ and $\mathrm{NW} 1 / 420,29 \mathrm{~S} ., 4 \mathrm{E} \ldots . .$. & Loa $1 \mathrm{SE}$ & $\begin{array}{l}\text { Smith and others (1963); SCP. } \\
\text { Fred Peterson (unpub. data, 1971); SCP. }\end{array}$ \\
\hline $\mathrm{U} 22 \ldots \ldots .$. & Fremont River ..................... & C SW1/4NE $1 / 46,29$ S., 7 E ..................... & Notom $1 \mathrm{SW}$.......................... & $\begin{array}{l}\text { Fred Peterson (unpub. data, } \\
\text { 1971); P, SCP. }\end{array}$ \\
\hline $\mathrm{U} 23 \ldots \ldots . .$. & Boulder .................................. & $\mathrm{NE}^{1} / 4 \mathrm{SW}^{1} / 4 \mathrm{SE}^{1} / 434,33 \mathrm{~S} ., 4 \mathrm{E} . . . \ldots \ldots . .$. & Boulder Town ..................... & $\begin{array}{l}\text { Fred Peterson (unpub. data. } \\
\text { 1971); SCP, ONC. }\end{array}$ \\
\hline $\mathrm{U} 24 \ldots \ldots .$. & The Post ................................. & 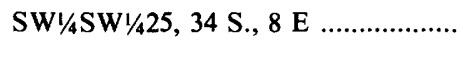 & Mount Pennell .................... & $\begin{array}{l}\text { Fred Peterson (unpub. data, } \\
\text { 1971); P. }\end{array}$ \\
\hline U25 ......... & Pine Creek ........................... & $\mathrm{NE}^{1} / 4 \mathrm{SW}^{1} / 4 \mathrm{SW}^{1} / 429,34 \mathrm{~S} ., 3 \mathrm{E}$ (un) .. & 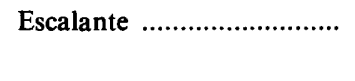 & $\begin{array}{l}\text { Fred Peterson (unpub. data, } \\
\text { 1971); P, SCP. }\end{array}$ \\
\hline $\mathrm{U} 26 \ldots \ldots . .$. & Escalante .............................. & SEl/4NW1/4SW1/422, 35 S., 4 E. (un) . & Ten Mile Flat ........................ & This report; SCP, ONC. \\
\hline U27 ........... & 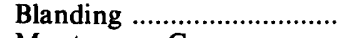 & 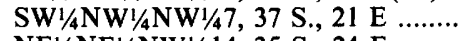 & Brushy Basin Wash ............ & This report; P, SCP. \\
\hline $\mathrm{U} 28 \ldots \ldots . .$. & Montezuma Canyon .......... & $\mathrm{NE}^{1 / 4 N^{1} 1 / 4 \mathrm{NW}^{1 / 414}, 35 \mathrm{~S} ., 24 \mathrm{E} . . . . . . .}$ & 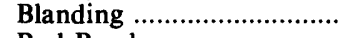 & Do. \\
\hline $\mathrm{U} 29 \ldots \ldots$ & 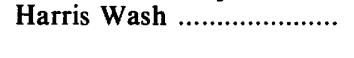 & $\mathrm{SW}^{1 / 4} \mathrm{SE}^{1 / 4} \mathrm{SW} 1 / 433,36 \mathrm{~S} ., 5 \mathrm{E}$ & Red Breaks ............................ & $\begin{array}{l}\text { Fred Peterson (unpub. data, } \\
\text { 1971); P. }\end{array}$ \\
\hline U30 ......... & Seep Flat ............................... & SW1/4NE1/46, 37 S., 5 E. (un) .............. & 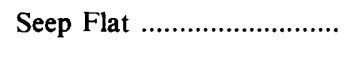 & $\begin{array}{l}\text { H. D. Zeller (unpub. data, } \\
\text { 1971); P, SCP. }\end{array}$ \\
\hline U31 ......... & Cedar City ............................. & $\mathrm{SE}^{1} / 4 \mathrm{SW} 1 / 4 \mathrm{SE} 1 / 412,36 \mathrm{~S} ., 11 \mathrm{~W} \ldots \ldots$. & Cedar City ............................. & $\begin{array}{l}\text { Fred Peterson (unpub. data, } \\
\text { 1971); P, SCP. }\end{array}$ \\
\hline
\end{tabular}


TABLE 1.-Location of control points and source of data used to construct restored sections and maps in this report-Continued

\begin{tabular}{|c|c|c|c|c|}
\hline $\begin{array}{c}\text { Locality } \\
\text { No. }\end{array}$ & Locality name & $\begin{array}{c}\text { Sec., T., R. } \\
\text { or } \\
\text { long } \mathrm{W} \text { and lat } \mathrm{N}\end{array}$ & Quadrangle & References and remarks \\
\hline
\end{tabular}

Utah-Continued

\begin{tabular}{|c|c|c|c|c|}
\hline U32 ......... & Bull Valley Gorge ............. & SW $1 / 4$ SW $1 / 4$ NW $1 / 427,38$ S., 3 W ...... & Bull Valley Gorge .............. & $\begin{array}{l}\text { Fred Peterson (unpub. data, } \\
\text { 1971); SCP, ONC. }\end{array}$ \\
\hline U33 ......... & Rush Beds ....................... & NW1/4SW1/4SW1/410, 39 S., 1 W. (un) & Slick Rock Bench ............. & Do. \\
\hline U34 .......... & Cat Pasture ..................... & C NW $1 / 4$ NW $1 / 426,38$ S., 6 E. (un) .... & Big Hollow Wash ............. & Do. \\
\hline U35 ........ & Hurricane Wash ............... & SW $1 / 4$ SE $1 / 4 N^{\prime} 1 / 413,39$ S., 8 E. (un) . & The Rincon $. . . . . . . . \ldots \ldots \ldots \ldots . . . .$. & $\begin{array}{l}\text { H. D. Zeller (unpub. data, } \\
\text { 1971); S. }\end{array}$ \\
\hline U36 ......... & Cave Point ........................ & $\mathrm{SE}^{1} / 4 \mathrm{NE}^{1} / 4 \mathrm{SW}^{1} 1 / 426,40 \mathrm{~S} ., 8 \mathrm{E}$. (un) .. & Sooner Bench ................... & $\begin{array}{l}\text { Fred Peterson (unpub. data, } \\
\text { 1971); P. }\end{array}$ \\
\hline U37 ......... & 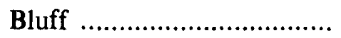 & NW $1 / 4$ NE $1 / 4$ NE $1 / 431,40$ S., 21 E ........ & Bluff ......... & This report; $P$. \\
\hline $\mathrm{U} 38 \ldots \ldots \ldots$ & Gunlock ............................. & SW $1 / 4$ SW $1 / 4 N^{\prime} 1 / 432,40$ S., 17 W ...... & Gunlock . & $\begin{array}{l}\text { Fred Peterson (unpub. data, } \\
\text { 1971); SCP, ONC. This } \\
\text { report, P. }\end{array}$ \\
\hline U39 ........ & 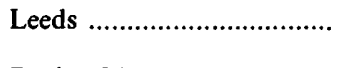 & NE $1 / 4 S^{2} 1 / 4 N^{1} / 434,40$ S., 14 W ....... & New Harmony ................... & $\begin{array}{l}\text { Fred Peterson (unpub. data, } \\
\text { 1971); SCP, ONC. }\end{array}$ \\
\hline U40 ......... & Pocket Mesa ..................... & NW $1 / 4$ NE $1 / 4$ NE $1 / 434,39$ S., 11 W ...... & Zion National Park .......... & Do. \\
\hline U41 ......... & Potato Hollow .................. & NE $1 / 4$ NE $1 / 4 N^{1} 1 / 420,40$ S., 10 W ....... & 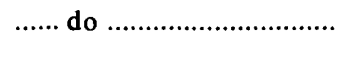 & $\begin{array}{l}\text { Fred Peterson (unpub. data, } \\
\text { 1971); P, SCP. }\end{array}$ \\
\hline U42 ......... & Zican Lodge ..................... & SW $1 / 4 S^{2} 1 / 4$ SW $^{1} / 416,41$ S., 9 W ... & Springdale NE ................ & Do. \\
\hline U43 ......... & Mount Carmel Junction ... & SW $1 / 4 S 1 / 4$ SE $1 / 425,41$ S., 8 W ... & 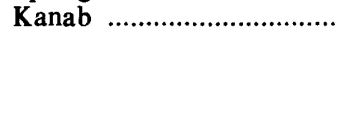 & $\begin{array}{l}\text { This report, P. Cashion } \\
\text { (1967, J3, J4); SCP. Fred } \\
\text { Peterson (unpub. data, 1971); } \\
\text { SCP. }\end{array}$ \\
\hline U44 …..... & Brown Canyon ................. & NE $1 / 4$ SE $1 / 4 N W 1 / 419,41$ S., 5 W ......... & 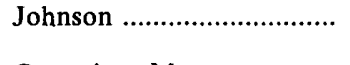 & $\begin{array}{l}\text { Fred Peterson (unpub. data, } \\
\text { 1971); P. SCP. }\end{array}$ \\
\hline U45 $\ldots \ldots . .$. & Rock Creek ....................... & $111^{\circ} 09^{\prime} 07^{\prime \prime}, 37^{\circ} 09^{\prime} 40^{\prime \prime} \quad \ldots \ldots \ldots \ldots \ldots \ldots . . . . . . . . . .$. & Cummings Mesa ............... & Do. \\
\hline U46 ......... & Sixtymile Point ................. & SE $1 / 4$ NE $1 / 4$ SW $1 / 46,42$ S., 9 E. (un) .... & Navajo Mountain .............. & $\begin{array}{l}\text { Fred Peterson (unpub. data, } \\
\text { 1971); SCP, ONC. }\end{array}$ \\
\hline U47 …..... & Cathedral Canyon ............. & $111^{\circ} 01^{\prime} 13^{\prime \prime}, 37^{\circ} 06^{\prime} 38^{\prime \prime} \ldots \ldots \ldots$ & Cummings Mesa ............... & $\begin{array}{l}\text { Fred Peterson (unpub. data, } \\
\text { 1971); S. }\end{array}$ \\
\hline U48 $\ldots \ldots . .$. & Wild Horse Bar ................ & $111^{\circ} 07^{\prime} 48^{\prime \prime}, 37^{\circ} 06^{\prime} 02^{\prime \prime}$ & 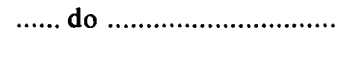 & $\begin{array}{l}\text { H. D. Zeller (unpub. data, } \\
\text { 1971); S. }\end{array}$ \\
\hline U49 ......... & West Cove Gulch ............. & 19,42 S., $1 \mathrm{~W} \ldots .$. & 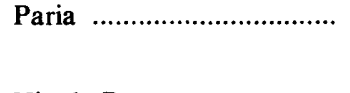 & $\begin{array}{l}\text { Fred Peterson (unpub. data, } \\
\text { 1971); SCP. Phoenix (1963, } \\
\text { p. 30); SCP. }\end{array}$ \\
\hline U50 .......... & Jacobs Tanks ....................... & 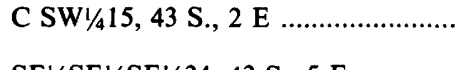 & Nipple Butte ......................... & $\begin{array}{l}\text { Fred Peterson (unpub. data, } \\
\text { 1971); P, SCP. }\end{array}$ \\
\hline U51 ......... & Gunsight Canyon .............. & $\mathrm{SE}^{1 / 4} \mathrm{SE}^{1 / 4} \mathrm{SE} 1 / 434,43 \mathrm{~S} ., 5 \mathrm{E} . . . \ldots \ldots \ldots . . . .$. & Gunsight Butte $\ldots . . \ldots \ldots \ldots \ldots$ & Do. \\
\hline
\end{tabular}

\begin{tabular}{|c|c|c|c|c|}
\hline \multicolumn{5}{|c|}{ Wyoming } \\
\hline W1 $1 \ldots \ldots . . .$. & Clarks Fork Canyon ......... & C W1/2NW1/45, 56 N., 103 W ........... & Deep Lake ......................... & $\begin{array}{l}\text { Imlay (1956, fig. 3, sec. 11); } \\
\text { SCP. This report; P. }\end{array}$ \\
\hline W2 & Sykes Mountain ................ & SE1/4SE1/4SW1/47, 57 N., 94 W .......... & Natural Trap Cave ............ & This report; $\mathbf{P}$. \\
\hline W3 $\ldots \ldots \ldots$ & Hulett $\ldots \ldots \ldots \ldots \ldots \ldots \ldots \ldots \ldots \ldots$ & SW $1 / 4$ NW $1 / 4$ SW $1 / 42,54$ N., 64 W ...... & Devils Tower ..................... & $\begin{array}{l}\text { Mapel and Bergendahl (1956, } \\
\text { p. 87, sec. 4); SCP, ONC. } \\
\text { This report; ONC. }\end{array}$ \\
\hline W4 $\ldots \ldots \ldots$ & Cody $\ldots \ldots \ldots \ldots \ldots \ldots \ldots \ldots \ldots$ & NE $1 / 43,52$ N., $102 \mathrm{~W}, \ldots \ldots \ldots \ldots \ldots \ldots \ldots$ & Cody $\ldots \ldots \ldots \ldots \ldots \ldots \ldots \ldots \ldots \ldots$ & $\begin{array}{l}\text { Imlay (1956, fig. } 3 \text {, sec. } 13) \\
\text { SCP. This report; } P \text {. }\end{array}$ \\
\hline W5 $\ldots \ldots \ldots$ & 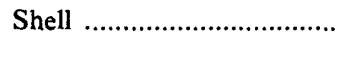 & 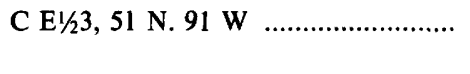 & Manderson NE ................. & $\begin{array}{l}\text { Imlay }(1956, \text { p. } 569) ; \text { SCP. } \\
\text { This report; P. }\end{array}$ \\
\hline W6 .......... & $\begin{array}{l}\text { North Fork Sayles } \\
\text { Creek. }\end{array}$ & SE $1 / 4$ SW $1 / 4$ SE $1 / 46,51$ N., 83 W .......... & Stone Mountain ................ & This report; P. SCP. \\
\hline W7 .......... & Sundance Mountain ......... & NE $1 / 425,51$ N., $63 \mathrm{~W}, \ldots \ldots \ldots \ldots \ldots \ldots \ldots$ & Sundance ............................. & $\begin{array}{l}\text { Mapel and Bergendahl (1956, } \\
\text { p. } 87 \text {, fig. } 2 \text {, sec. 8); SCP, } \\
\text { ONC. }\end{array}$ \\
\hline W8 .......... & Dry Muddy Creek ............. & NE $1 / 4 \mathrm{SW}^{1} / 42,48 \mathrm{~N} ., 83 \mathrm{~W} \ldots \ldots \ldots \ldots \ldots$ & Klondike Ranch ................ & $\begin{array}{l}\text { Hose (1955, p. 106); SCP, ONC. } \\
\text { This report; P. }\end{array}$ \\
\hline W9 ............ & Tensleep ............................... & 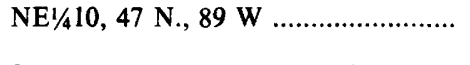 & Wild Horse Hill ................. & $\begin{array}{l}\text { Imlay }(1956, \text { p. } 570) ; \mathrm{SCP} \\
\text { ONC. This report; P. }\end{array}$ \\
\hline W10 & Ramsbottom Ranch ......... & SW $1 / 4 N^{1} / 4 S^{1} 1 / 423,46$ N., 83 W $\ldots .$. & Mayoworth ........................ & $\begin{array}{l}\text { Peterson (1954, p. } 481 \text {, unit } \\
\text { 2); ONC. This report; P. } \\
\text { SCP. }\end{array}$ \\
\hline
\end{tabular}


TABLE 1.-Location of control points and source of data used to construct restored sections and maps in this report-Continued

\begin{tabular}{lccc}
\hline $\begin{array}{c}\text { Locality } \\
\text { No. }\end{array}$ & Locality name & $\begin{array}{c}\text { Sec., } \mathrm{T} ., \mathrm{R} . \\
\text { or } \\
\text { long } \mathrm{W} \text { and lat } \mathrm{N}\end{array}$ & Quadrangle \\
\hline
\end{tabular}

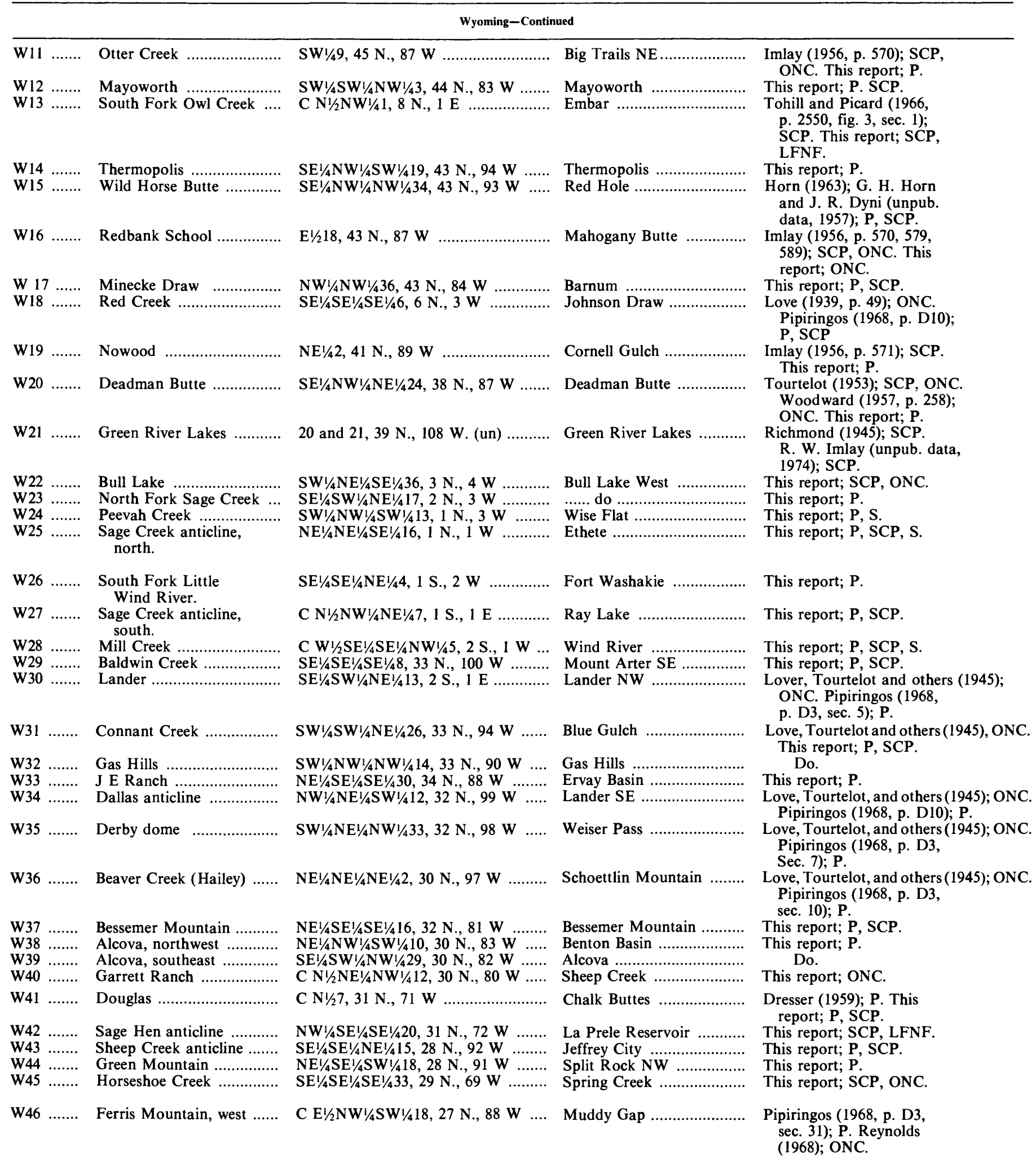


TABLE 1.-Location of control points and source of data used to construct restored sections and maps in this report-Continued

\begin{tabular}{|c|c|c|c|c|}
\hline $\begin{array}{l}\text { Locality } \\
\text { No. }\end{array}$ & Locality name & $\begin{array}{c}\text { Sec., } T \text {., } R \text {. } \\
\text { or } \\
\text { long } W \text { and lat } N\end{array}$ & Quadrangle & References and remarks \\
\hline \multicolumn{5}{|c|}{ Wyoming-Continued } \\
\hline $\begin{array}{l}\text { W47 } \\
\text { W48..... } \\
\text { W4..... }\end{array}$ & $\begin{array}{l}\text { Ferris Mountain, east ....... } \\
\text { Freezeout Hills, } \\
\text { northeast. }\end{array}$ & 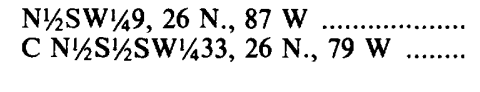 & 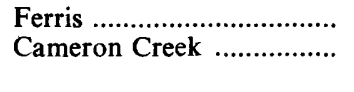 & $\begin{array}{l}\text { This report; P. } \\
\text { This report; P, SCP. }\end{array}$ \\
\hline $\begin{array}{l}\text { W49 } \\
\text { W50........ }\end{array}$ & $\begin{array}{l}\text { Sheep Creek } \\
\text { Hurt Creek }\end{array}$ & $\begin{array}{l}\text { NW1/4SW1/4NE1/435, } 26 \text { N., } 76 \mathrm{~W} \\
\mathrm{NE}^{1 / 4} \mathrm{NW}^{1 / 4} \mathrm{NW} 1 / 424,25 \mathrm{~N} ., 85 \mathrm{~W} \\
. . . .\end{array}$ & 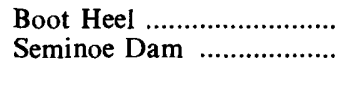 & $\begin{array}{l}\text { This report; SCP, LFNF. } \\
\text { Pipiringos (1968, p. D3, } \\
\text { sec. 33); P. }\end{array}$ \\
\hline W51 ........ & Sips Creek ........................ & $\mathrm{NE}^{1 / 4} \mathrm{NE} 1 / 4 \mathrm{NW} 1 / 424,25 \mathrm{~N} ., 84 \mathrm{~W} \ldots . .$. & Seminoe Dam NE .............. & $\begin{array}{l}\text { Pipiringos (1968, p. D3, sec. } \\
\text { 35); SCP, ONC. }\end{array}$ \\
\hline W52 ........ & Mud Spring .......................... & $\mathrm{N} 1 / 2 \mathrm{~N} 1 / 2 \mathrm{SE} 1 / 434,25 \mathrm{~N} ., 81 \mathrm{~W} . . . \ldots \ldots \ldots . . .$. & T E Ranch ............................ & $\begin{array}{l}\text { Pipiringos (1968, p. D3, sec. } \\
\text { 39); P, SCP. }\end{array}$ \\
\hline W53 ....... & Freezeout Hills, east .......... & $\mathrm{NE} 1 / 4 \mathrm{NE} 1 / 4 \mathrm{SE} 1 / 430,25 \mathrm{~N} ., 78 \mathrm{~W} \ldots \ldots .$. & T B Ranch .............................. & $\begin{array}{l}\text { Pipiringos (1968, p. D20); } \\
\text { ONC, SCP. }\end{array}$ \\
\hline W54 ........ & $\begin{array}{l}\text { Freezeout Hills, } \\
\text { southwest. }\end{array}$ & $\mathrm{SE}^{1 / 4} \mathrm{NE}^{1 / 4} \mathrm{NE}^{1 / 426}, 24 \mathrm{~N} ., 80 \mathrm{~W} \ldots \ldots .$. & Windy Hill ............................. & $\begin{array}{l}\text { Pipiringos (1968, p. D14); } \\
\text { ONC, SCP. }\end{array}$ \\
\hline W55 ........ & Bell Springs ........................ & C W $1 / 2 N^{\prime} 1 / 4 S^{1} 1 / 49,23$ N., 88 W $\ldots . .$. & Rawlins Peak ...................... & $\begin{array}{l}\text { Pipiringos (1968, p. D18, } \\
\text { fig. 14; p. D5, fig. 4); P. }\end{array}$ \\
\hline W56 ........ & Rawlins, northeast ............ & $\mathrm{NW}^{1} / 4 \mathrm{NE}^{1} / 4 \mathrm{NE}^{1} / 436,22 \mathrm{~N} ., 87 \mathrm{~W} . . . .$. & Rawlins ................................ & $\begin{array}{l}\text { Pipiringos (1968, p. D3, sec. } \\
\text { 58); P, SCP. }\end{array}$ \\
\hline $\begin{array}{l}\text { W57 } \ldots \ldots . . \\
\text { W } 58 \ldots \ldots .\end{array}$ & 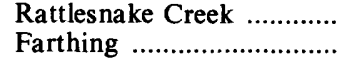 & 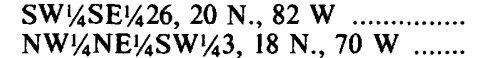 & $\begin{array}{l}\text { Rattlesnake Pass } \ldots \ldots \ldots \ldots \ldots \ldots \\
\text { Farthing } \ldots \ldots \ldots \ldots \ldots \ldots \ldots \ldots \ldots \ldots\end{array}$ & $\begin{array}{l}\text { This report; SCP, LFNF. } \\
\text { This report; SCP, ONC. }\end{array}$ \\
\hline W59 ....... & Littlefield Creek ................. & C W $1 / 2 N^{1} 1 / 4 N^{1} 1 / 411,17$ N., 89 W (un) & Pole Gulch ........................ & $\begin{array}{l}\text { Pipiringos (1972, p. 20, sec. } \\
\text { 61); SCP, ONC. }\end{array}$ \\
\hline W $60 \ldots .$. & Sage Creek Basin .............. & $\mathrm{SW}^{1} / 4 \mathrm{NE}^{1 / 4 \mathrm{NE}^{1} / 427,17 \mathrm{~N} ., 88 \mathrm{~W} . . . . . .}$ & Pine Grove Ranch ............. & $\begin{array}{l}\text { Pipiringos (1972, p. } 20, \text { sec. } \\
\text { 62); SCP, ONC. }\end{array}$ \\
\hline W $61 \ldots \ldots$ & Horse Creek ......................... & C SW1/4NE1/4SE1/434, 17 N., 70 W .... & Horse Creek .. & This report; P, SCP. \\
\hline W62 ….... & Mesa Mountain ................. & C SW1/4NE $1 / 4$ SW $1 / 435,16$ N., 70 W .. & Islay & Do. \\
\hline W63 ........ & Big Sandstone Creek ......... & $\mathrm{E}^{1} / 2 \mathrm{SE} 1 / 4 \mathrm{NE} 1 / 419,14 \mathrm{~N} ., 87 \mathrm{~W} \ldots \ldots \ldots .$. & Singer Peak ............................ & $\begin{array}{l}\text { Pipiringos (1972, p. 20, sec. } \\
\text { 63); P, SCP. }\end{array}$ \\
\hline W64 '........ & Roaring Fork ..................... & $\mathrm{SW}^{1} / 4 \mathrm{SE}^{1 / 4} \mathrm{NE}^{1} / 48,12 \mathrm{~N} ., 86 \mathrm{~W} \ldots \ldots \ldots$ & Fletcher Peak ........................ & $\begin{array}{l}\text { Pipiringos (1972, p. 20, sec. } \\
\text { 65); P. }\end{array}$ \\
\hline W65 ........ & Red Mountain ...................... & NE $1 / 4 \mathrm{SW}^{1} / 4 \mathrm{NW}^{1 / 4} 16,12 \mathrm{~N} ., 76 \mathrm{~W} \ldots .$. & Jelm Mountain ..................... & Pipiringos (1957, p. 58); P. \\
\hline
\end{tabular}

\section{UNCONFORMITIES}

Unconformities are the most rigorous expression of the law of superposition. A unit above an unconformity cannot be the time equivalent of any part of a unit below an unconformity. On the other hand, a seemingly straightforward superposition of conformable units does not necessarily indicate that the unit on top is entirely younger than the unit on the bottom, except at the point of observation (fig. 3).

Some of our colleagues independently questioned the concept illustrated by units $C$ and $D$ in figure 3 . They intuitively believed that if the unconformity between these two units were time-transgressive that some part of $\mathrm{C}$ might equal some part of $\mathrm{D}$. We agree that theoretically this might be possible under a very limited set of circumstances: (1) In figure 3 the uplift must travel from left to right like a crustal wave. (2) the amplitude of the uplift must progressively diminish in such a way that a line joining successive crests coincides with the erosion surface and (3) the area behind the moving crest of the uplift must subside and remain below erosional base level in order to preserve any sediments eroded from the receding crest. We doubt that such a sequence of events occurred in areas of relative crustal stability such as the Western Interior of the United States.

Unconformities show two conspicuously different relationships to paleo-highs or positive areas. An unconformity bounding a paleo-high is preserved where the paleo-high is gradually buried by a conformable sequence of rocks (fig. $4 A$ ). On the other hand, younger unconformities partially destroy older unconformities at paleo-highs (fig. 4B). Consequently, a series of progressively younger unconformities result in a composite erosion surface which bounds the paleo-high. This erosion surface consists of the preserved remnants of the pre-existing unconformities.

Two small isolated areas in Wyoming of the middle part and of the Hulett Sandstone Member, both of the Sundance Formation, and a larger area in southwestern Colorado of younger Jurassic rocks (map $A, \mathrm{pl} .1$ ), are examples of conformable onlap of paleo-highs that are bounded by a single surface, as illustrated in figure $4 A$. Because the surface of erosion, in this case the $\mathrm{J}-2$ surface, is preserved in those areas, they are given the color of the stratigraphic units by which they are buried (map $A$, pl. 1). 

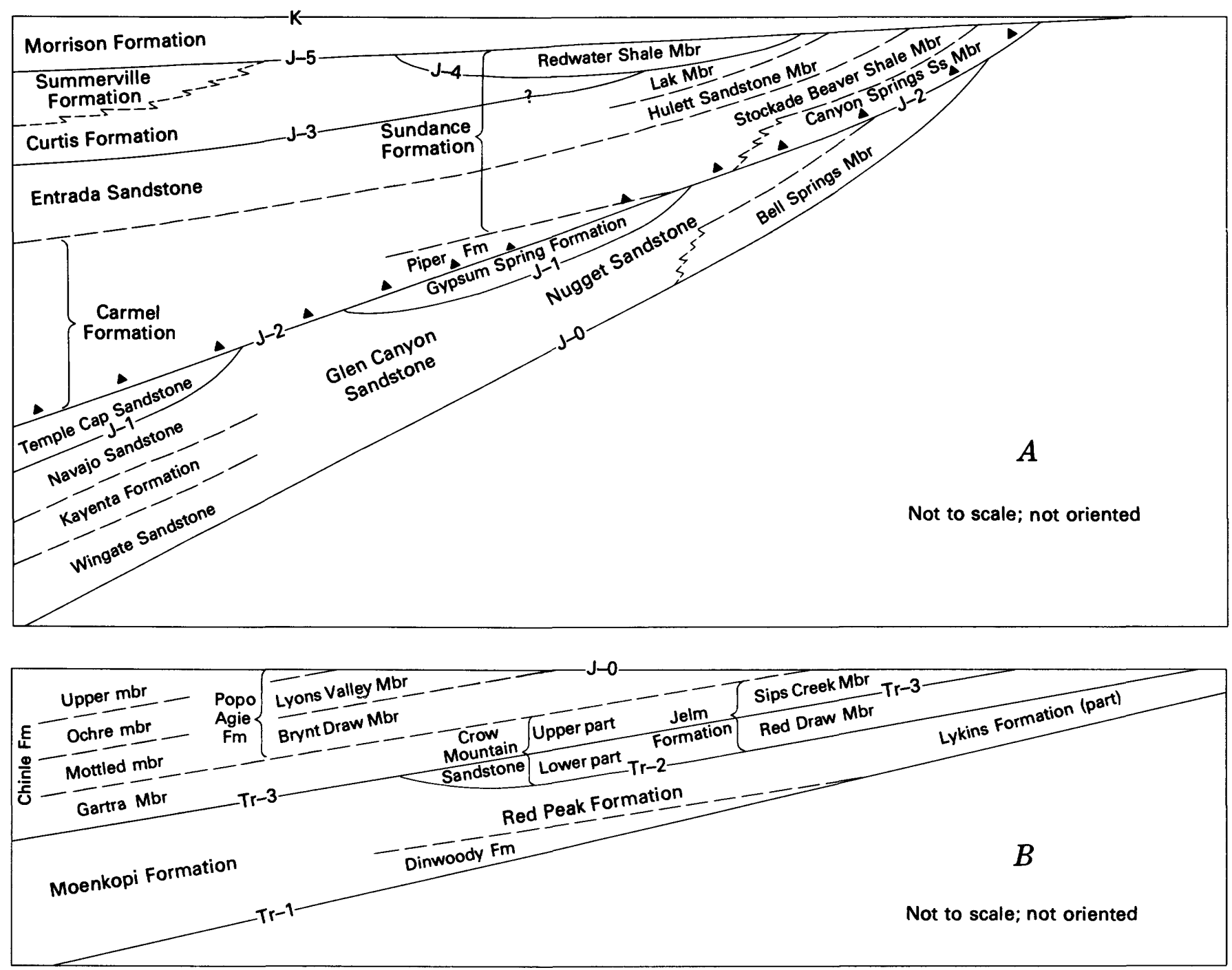

FIGURE 2.-Diagram illustrating the arrangement and relationships of the several unconformities to each other and to the enclosed sedimentary wedges, and the unconformity numbering system used in this report. $A$, Rocks at the end of Jurassic time. $B$, Rocks at the end of Triassic time. Unconformities are shown by solid lines: conformable contacts are dashed; triangles denote chert pebbles.
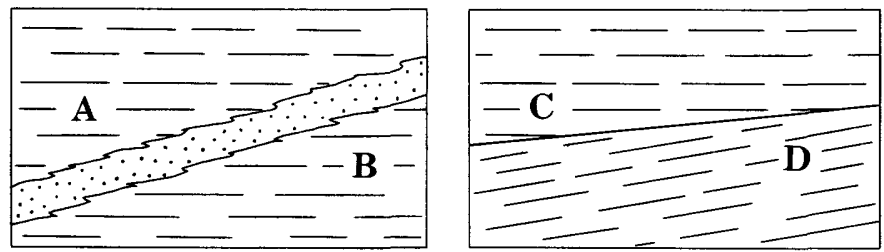

FIGURE 3.-Diagram illustrating two corollaries of the law of superposition. Superimposed but discontinuous units A and B are partly contemporaneous; superimposed and seemingly laterally continuous units $\mathrm{C}$ and $\mathrm{D}$ are separated by an unconformity (solid line) and are nowhere contemporaneous. In the diagrams hypothetical time lines parallel the pattern of dashes.

By contrast, a persistent positive area whose upper reaches ultimately formed ancient Belt Island in Montana is an example of a paleo-high bounded by a composite erosion surface such as that shown in figure $4 B$, but more complex.

Those parts of Belt Island now overlain by the Swift and Morrison Formations are left uncolored on map $A$ of plate 1 because the $\mathrm{J}-2$ unconformity has been destroyed in those areas. Similarly, small areas in Colorado and at the Colorado-New Mexico State line are left uncolored where the $\mathbf{J}-2$ unconformity has been breached by erosion that preceded deposition of the Morrison Formation and the Dakota Sandstone.

Most of the Triassic and Jurassic rocks in the Western Interior are unfossiliferous, sparsely fossiliferous, or lack diagnostic fossils. The correlation and age of these rocks have been inferred by their relation to the rocks of known age, stratigraphic position, and lithologic similarities. It is 
TABLE 2.--Lithologic descriptions of stratigraphic units

[Only dominant or characteristic lithologies are given. All units are marine except as noted. Map symbols are those used on plate 1 . See plate $I$ for correlation of units and relation of units to unconformities discussed in this report]

\begin{tabular}{|c|c|}
\hline $\begin{array}{c}\text { Map } \\
\text { symbol }\end{array}$ & Unit and description \\
\hline
\end{tabular}

Upper, Lower(?), and Lower Cretaceous

Kd.............. Dakota Sandstone-Tan, brown, and gray sandstone, conglomeratic sandstone, and quartzite; partly fluviatile and paludal.

\section{Lower Cretaceous}

Ke .............. Ephraim Conglomerate-Gray sandstone, quartzite, and limestone interbedded with red conglomerate, siltstone, and limestone; fluviatile and lacustrine.

\begin{tabular}{l} 
Upper Jurassic \\
\hline Jm.............. Morrison Formation-Variegated fluvial mudstone and \\
$\begin{array}{l}\text { sandstone, gray-green lacustrine shale, and thin limestone } \\
\text { beds. }\end{array}$
\end{tabular}

Upper and Middle Jurassic

Sundance Formation

Jsw.............. Windy Hill Sandstone Member (Upper Jurassic)-Grayish-white to grayish-yellow and brownish-gray thin-bedded limy ripple-marked sandstone containing grayish-green and dark-gray shale partings.

Jsr............... Redwater Shale Member (Upper Jurassic)-Greenish-gray clayey siltstone, clay shale, and limy coquinoid sandstone or sandy coquinoid limestone.

Jsp .............. Pine Butte Member (Middle Jurassic)-Greenish-white limy sandstone and intercalated siltstone and grayish-to olive-green clay shale.

Js1 ............... Lak Member (Middle Jurassic)-Reddish-brown siltstone, sandy siltstone, and silty sandstone.

Jsh .............. Hulett Sandstone Member (Middle Jurassic)-Greenishto yellowish-white sandstone and gray-green clay shale.

Jsm ............. Middle part (Middle Jurassic) - Pine Butte, Lak, and Hulett Members.

Jss............... Stockade Beaver Shale Member (Middle Jurassic)-Greenish-gray clay shale and siltstone.

Jsc.............. Canyon Springs Sandstone Member (Middle Jurassic) Sandstone, mostly crossbedded; usually contains lesser amounts of massive sandstone and flat-bedded ripple-marked oolitic sandstone near the top.

Js ............... Swift Formation

Upper part (Upper Jurassic) - Flaggy ripple-marked sandstone containing abundant black-gray fissle shale lenses.

Lower part (Upper and Middle Jurassic)-Dark-gray noncalcareous shale.

Stump Sandstone

Jstu ............ Upper part (Upper Jurassic) - Gray shale, claystone, and limestone.

Jstl.............. Lower part (Middle Jurassic)-Gray to greenish-gray sandstone; some interbedded siltstone and shale.

\begin{tabular}{c} 
Middle Jurassic \\
\hline Jr ............... Rierdon Formation-Alternating gray limy shale and \\
limestone.
\end{tabular}

TABLE 2.-Lithologic descriptions of stratigraphic units-Continued

\section{Map \\ symbo}

J

$\mathrm{Jp}$
Middle Jurassic-Continued

Jsa.............. Sawtooth Formation

Upper part-Highly calcareous siltstone.

Middle part-Dark-gray shale containing a few thin dark limestone layers.

Lower part-Fine-grained sandstone.

Piper Formation

Upper part-Red beds and gypsum.

Middle part-Gray shale, limestone, and dolomite.

Lower part-Red beds and gypsum

Jpr ............. Preuss Sandstone-Red siltstone and sandstone.

Jtc............... Twin Creek Limestone

Jtgc ............. Giraffe Creek Member-Gray silty to sandy ripplemarked thin-bedded limestone and sandstone; some thicker beds of oolitic sandy limestone.

Jtl............... Leeds Creek Member-Light-gray soft dense shaly splintery limestone; some oolitic silty or sandy ripple-marked limestone.

Jtw .............. Watton Canyon Member-Gray dense compact brittle even-bedded medium- to thin-bedded limestone; basal bed generally massive and oolitic.

Jtb.............. Boundary Ridge Member--Red, green, and yellow soft siltstone interbedded with silty to sandy or oolitic limestone.

Jtr ............... Rich Member-Medium-gray shaly limestone.

Jts.............. Sliderock Member-Grayish-black medium- to thin-bedded limestone. In Wyoming, basal beds are oolitic; in Idaho, sandy, crossbedded, and pebbly; in Utah, sandy and oolitic.

Jtcl............. Lower part-Boundary Ridge, Rich, and Sliderock Members.

Jtg............... Gypsum Spring Member-Red to yellow siltstone and silty claystone interbedded with brecciated cherty limestone.

Jsu .............. Summerville Formation-Interbedded reddish-brown siltstone and shale; lesser amounts of tan and grayish-orange sandstone.

Jcr.............. Curtis Formation-Light-gray glauconitic oolitic sandstone containing clay shale partings near top; grayish-green clay shale and light-gray thin-bedded ripple-marked sandstone near base.

Jy............... Younger Jurassic rocks-In Arizona and New Mexico, includes the Summerville Formation, Bluff Sandstone (partly eolian), and Cow Springs Sandstone (eolian); in Colorado, includes the Wanakah Formation (partly lacustrine) and Junction Creek Sandstone (partly eolian).

Jt................ Todilto Limestone-Light-gray dense and crystalline limestone; gypsum locally at top; probably lacustrine.

Je.............. Entrada Sandstone-Red earthy siltstone and orange, buff, and white sandstone; partly eolian; locally in southeast Utah and adjacent areas divisible into the Moab Member above and the Slick Rock Member below.

Jed .............. Dewey Bridge Member-Reddish-brown siltstone, sandy siltstone, and silty sandstone.

Jc............... Carmel Formation

Jcw.............. Winsor Member-White and banded white-and-red sandstone.

Jcg............. Gypsiferous member-Massive gypsum and gray fossiliferous limestone. 
TABLE 2.-Lithologic descriptions of stratigraphic units-Continued

\begin{tabular}{cc}
$\substack{\text { Map } \\
\text { symbol }}$ & Unit and description \\
\hline & Middle Jurassic-Continued
\end{tabular}

Jcu.............. Upper part-Dark-reddish-brown shale and reddishorange and yellowish-gray sandstone.

Jcb .............. Banded member-Sandstone, banded pale red and white.

Jcj ............... Judd Hollow Tongue-Reddish-brown limy sandstone and siltstone.

Jcl ............... Lower part-Gray fossiliferous limestone, red, green, and white sandstone, gray and red shale, and gypsum.

Jpa .............. Page Sandstone-Reddish-orange to reddish-brown sandstone; eolian.

Jpat............. Thousand Pockets Tongue-Reddish-brown to lightgray crossbedded sandstone.

Jpah............ Harris Wash Tongue-Reddish-brown and grayishyellow crossbedded sandstone.

Jne .............. Nesson Formation of Nordquist (1955)

Upper part-Light-brown dolomite containing thin lenses of red shale.

Middle part-Variegated shale.

Lower part-Interbedded anhydrite, red shale, and gray earthy dolomite.

Jg................ Gypsum Spring Formation

Upper part-Reddish-brown claystone and gray fossiliferous limestone.

Middle part-Gypsum.

Lower part-Reddish-brown siltstone.

Jtca .............. Temple Cap Sandstone

White Throne Member-Light-red, tan, white, and yellow massive and crossbedded cliff-forming sandstone; mainly eolian.

Sinawava Member-Red, gray, and brown irregularly bedded shaly calcareous sandstone, siliceous limestone, and gypsum.

\begin{tabular}{|c|c|}
\hline \multicolumn{2}{|r|}{ Jurassic and Triassic } \\
\hline Jkn.................. & Sandstone (Jurassic(?) and Triassic(?)) \\
\hline Jknu................ & $\begin{array}{l}\text { Upper part-Yellowish-white to pink crossbedded } \\
\text { sandstone; some reddish-brown silty sandstone; eolian. }\end{array}$ \\
\hline 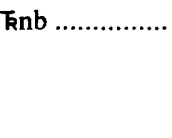 & $\begin{array}{l}\text { Bell Springs Member (Upper Triassic(?)-Red and gray } \\
\text { ripple-marked sandstone and red, green, and } \\
\text { pale-purplish-red to pale-red siltstone and shale; partly } \\
\text { fluviatile. }\end{array}$ \\
\hline 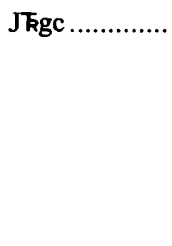 & $\begin{array}{l}\text { Glen Canyon Sandstone (Jurassic and Triassic)- } \\
\text { Gray, orange, brown, yellow, pink, and white } \\
\text { parallel-bedded and crossbedded dominantly eolian } \\
\text { sandstone; includes equivalents of Navajo Sandstone, } \\
\text { Kayenta Formation, and Wingate Sandstone which } \\
\text { belong to the Glen Canyon Group on the Colorado } \\
\text { Plateau. }\end{array}$ \\
\hline 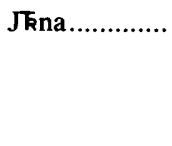 & $\begin{array}{l}\text { Navajo Sandstone (Jurassic and Triassic(?))-Grayish- } \\
\text { orange to pale-reddish-brown crossbedded eolian } \\
\text { sandstone; light-gray thin cherty lacustrine limestone beds } \\
\text { are common. }\end{array}$ \\
\hline $\mathrm{Fk} \ldots \ldots \ldots \ldots . . . . . .$. & $\begin{array}{l}\text { Kayenta Formation (Upper Triassic(?))-Pale-red } \\
\text { and purple sandstone, siltstone, and shale; fluviatile. }\end{array}$ \\
\hline mo ....... & $\begin{array}{l}\text { Moenave Formation (Upper Triassic(?))-Reddish- } \\
\text { orange and brown lenticular sandstone, siltstone, and } \\
\text { shale; fluviatile. }\end{array}$ \\
\hline
\end{tabular}

TABLE 2.-Lithologic descriptions of stratigraphic units-Continued

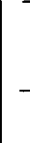

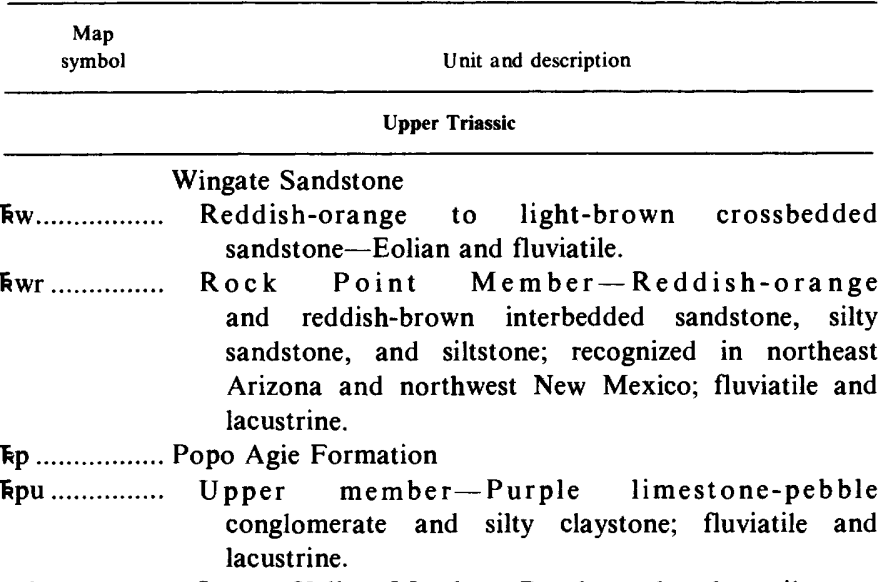

Kpl .............. Lyons Valley Member-Purple and ochre siltstone, analcime-rich claystone, and analcimolite; lacustrine.

Kpb .............. Brynt Draw Member-Pale- to purplish-red silty claystone and locally mottled gray and grayish-yellow silty sandstone; fluviatile and lacustrine.

Jelm Formation

Kjs................ Sips Creek Member-Greenish-white and yellow massive crossbedded cliff-forming sandstone; fluviatile and eolian. Grades laterally into reddish-brown ledge-forming sandstone and slope-forming siltstone; fluviatile and lacustrine.

Fjr............... Red Draw Member-Reddish-brown shale, siltstone and sandstone interbedded with some greenish-gray limy ripple-marked ledge-forming siltstone; fluviatile; grades laterally into salmon-red crossbedded eolian sandstone.

Crow Mountain Sandstone

Kcmu............ Upper part-White to reddish-brown sandstone and siltstone; minor amounts of pale-red and green shale; fluviatile.

Kcml.............. Lower part-Salmon-red to reddish-brown crossbedded sandstone; minor amounts of medium-bedded ripple-marked sandstone and siltstone; some sandy clay shale.

Fc................. Chinle Formation

Kcs ............... Siltstone member-Reddish-orange parallel-bedded and crossbedded siltstone and sandstone; limestone nodules common in lower part; fluviatile and lacustrine.

Kcoc............. Ochre siltstone member-Ochre and red structureless siltstone and clayey siltstone; minor amounts of silty claystone; lacustrine.

Kcmt............. Mottled member-Purple and red mudstone and mottled siltstone and sandstone; fluviatile and lacustrine.

Kcg.............. Gartra Member-Light-gray and purplish-red sandstone, grit, and conglomeratic sandstone; fluviatile.

Kco............... Owl Rock Member-Pink and red shale, reddishorange siltstone, and interbedded ledge-forming bluishand greenish-gray cherty limestone; lacustrine and fluviatile.

Kcpo............ Petrified Forest, Monitor Butte, and Shinarump Members and equivalent rocks-Variegated shale and siltstone, reddish-purple to grayish-red mudstone and siltstone, and light-gray and tan coarse-grained sandstone and conglomeratic sandstone; lacustrine and fluviatile. 
TABLE 2.-Lithologic descriptions of stratigraphic units-Continued

$\begin{gathered}\text { Map } \\ \text { symbol }\end{gathered}$
Upper Triassic-Continued

\begin{tabular}{|c|c|}
\hline Ћcu............... & $\begin{array}{l}\text { Upper part of the Petrified Forest Member and } \\
\text { younger parts of the formation. }\end{array}$ \\
\hline kel................ & $\begin{array}{l}\text { Lower part of the Petrified Forest Member and older } \\
\text { parts of the formation. }\end{array}$ \\
\hline
\end{tabular}

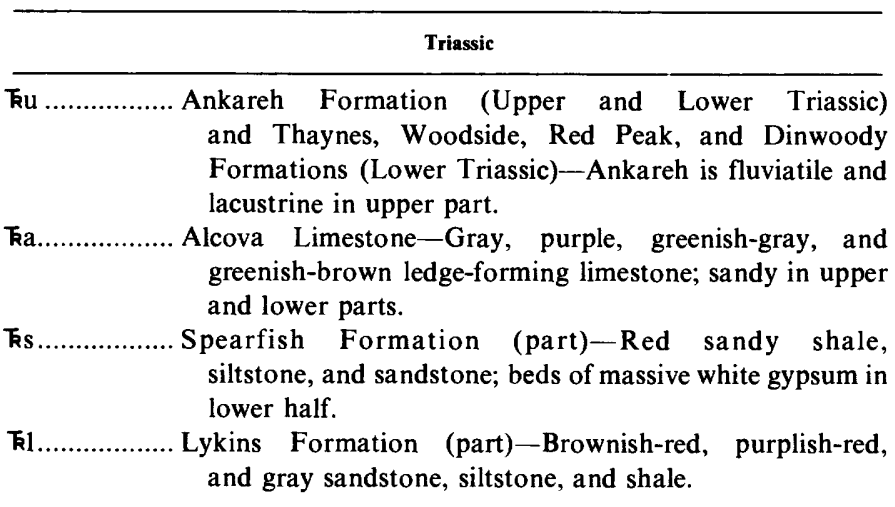

Middle(?)! and Lower Triassic
Km ............... Moenkopi Formation-Red and reddish-brown siltstone and silty sandstone, yellow and light-gray sandstone and mudstone, and light-gray limestone.
Fmu ............. Upper part-That part above the Sinbad Limestone Member.
Kms.............. Sinbad Limestone Member-Light-gray sandy limestone.
Kml .............. Lower part-That part below the Sinbad Limestone Member.

\section{Lower Triassic}

Fr................Red Peak Formation-Pale- to moderate-reddishbrown siltstone; some thin interbedded yellowish-gray sandstone.

Fd ............... Dinwoody Formation-Gray and olive-gray shaly siltstone and shale; thin brown limestone beds near base.

In Colorado Plateau only.

precisely in such rocks that unconformities are most useful for correlation. We have found that in the cratonic shelf area of the Western Interior unconformities in Triassic and Jurassic rocks are widespread surfaces that prior to burial resembled smooth peneplains. All are truncated entirely or partly by a younger unconformity. Whether the unconformities originally disappeared westward into conformable geosynclinal sequences is unknown; such sequences are not present within or near the study area. Formations bracketed by these widespread unconformities in the lower and middle Mesozoic rocks of the Western Interior are shown in correlation of units on plate 1 .

Estimates of the length of time required for uplift and erosion of an unconformity are arrived at by (1) noting the youngest beds under and the oldest beds over the unconformity anywhere within the area in which the unconformity occurs; (2) determining the European stages represented by these stratigraphic units as recognized in the Western Interior United States by Imlay (1947, 1952a, 1952b, 1956, 1964, 1968, 1973) and by Reeside and others (1957); and (3) using the time values assigned to the stages in the Phanerozoic time scale as summarized by the Geological Society of London (1964, p. 261) and as modified for the Jurassic Period by van Hinte (1976). Similarly, the length of time required for burial of an erosion surface is estimated by noting the oldest and youngest units over the unconformity. The series, stages, and time scales for the Jurassic and Triassic Systems used in this report are shown in figure 5.

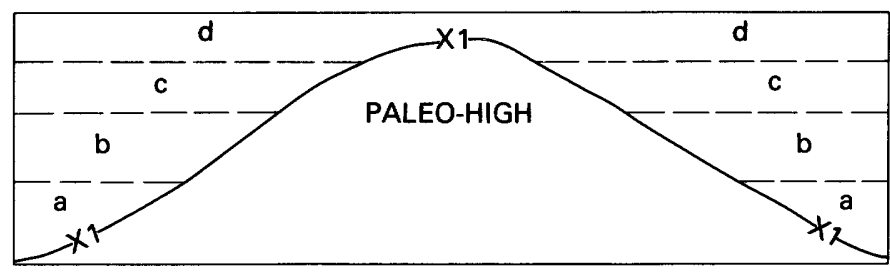

$A$

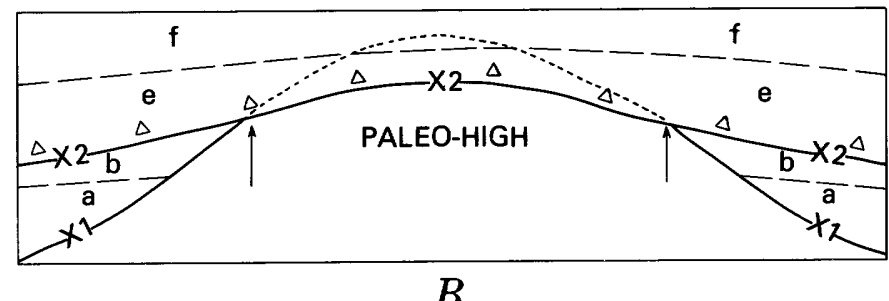

FIGURE 4.- Relationship of unconformities to a paleo-high. $A$, preservation of hypothetical unconformity $\mathrm{X} 1$ (solid line) by a conformable sequence of strata. $B$, partial destruction of unconformity X1 (between arrows) by younger unconformity $\mathrm{X} 2$ at a paleo-high. The former position of the destroyed $\mathrm{XI}$ unconformity is shown by the dotted line, and the paleo-high is now bounded by a composite surface consisting of the $\mathrm{X} 1$ and $\mathrm{X} 2$ unconformities. $\mathrm{a}-\mathrm{f}$, strata.

\section{TRIASSIC UNCONFORMITIES}

\section{TR-1 UNCONFORMITY}

The Tr-1 unconformity marks the base of the Moenkopi and equivalent formations throughout wide areas of the Western Interior. In most of the area, the Tr-1 unconformity is at the top of Permian rocks of lithologic diversity but of about the same age. In parts of Montana (McKee and others, 1959, pl. 2) and in parts of Colorado, the Tr-1 unconformity truncates pre-Permian rocks. In the Williston basin of eastern Montana and western North Dakota, the Tr-1 unconformity seems to correlate with an unconformity at the base of the Permian Pine Salt of Zieglar (1956, p. 174, fig. 4). In the southeastern part of the Black Hills of South Dakota, at Buffalo Gap, about $13 \mathrm{~km}$ northeast of Hot Springs, the 


\begin{tabular}{|c|c|c|}
\hline & & $\begin{array}{l}\text { AGE OF BASE } \\
\text { (m.y.) }\end{array}$ \\
\hline \multicolumn{2}{|c|}{ CRETACEOUS } & 135 \\
\hline \multirow{3}{*}{$\begin{array}{l}\text { UPPER } \\
\text { JURASSIC }\end{array}$} & Tithonian & 141 \\
\hline & Kimmeridgian & 143 \\
\hline & Oxfordian & 149 \\
\hline \multirow{3}{*}{$\begin{array}{l}\text { MIDDLE } \\
\text { JURASSIC }\end{array}$} & Callovian & 156 \\
\hline & Bathonian & 165 \\
\hline & Bajocian & 174 \\
\hline \multirow{4}{*}{$\begin{array}{l}\text { LOWER } \\
\text { JURASSIC }\end{array}$} & Toarcian & 178 \\
\hline & Pliensbachian & 183 \\
\hline & Sinemurian & 189 \\
\hline & Hettangian & 192 \\
\hline \multirow{3}{*}{$\begin{array}{l}\text { UPPER } \\
\text { TRIASSIC }\end{array}$} & Rhaetian & 195 \\
\hline & Norian & 200 \\
\hline & Carnian & [205] \\
\hline \multirow{2}{*}{$\begin{array}{l}\text { MIDDLE } \\
\text { TRIASSIC }\end{array}$} & Ladinian & 210 \\
\hline & Anisian & [215] \\
\hline \multirow{2}{*}{$\begin{array}{l}\text { LOWER } \\
\text { TRIASSIC }\end{array}$} & Olenekian & 220 \\
\hline & Induan & [225] \\
\hline
\end{tabular}

FIgURE 5.-Series, stages, and time-scales of the Jurassic and Triassic Systems used in this report. Ages given in millions of years for bases of stages of the Jurassic are from van Hinte (1976, fig. 3). Ages given in millions of years for bases of stages of the Triassic shown in brackets are from the Geological Society of London(1964, p. 261); other ages are our interpolations.

$\operatorname{Tr}-1$ unconformity probably is the contact between unit 1 and the overlying unit 2 of the Spearfish Formation as described by P. J. Lewis and H. D. Hadley (in Reeside and others, 1957, p. 1485). P. J. Lewis and H. D. Hadley correlated their unit 2 in the Black Hills area with the Lower Triassic Dinwoody Formation west of the Black Hills and their unit 1 with the upper part of the Permian Phosphoria Formation in the Bighorn Basin of Wyoming. In western
Colorado, eastern Utah, eastern Arizona, and New Mexico, the $\operatorname{Tr}-1$ unconformity is beveled out by the $\operatorname{Tr}-3$ unconformity. In central Montana and in South Dakota, and southward, the Tr-1 unconformity is truncated by the $\mathrm{J}-2$ unconformity. In the Williston basin, the $\mathrm{Tr}-1$ surface is probably truncated by the $\mathrm{Tr}-2$ unconformity.

The Tr-1 unconformity is described in numerous reports as a conspicuous erosion surface in parts of Nevada, Utah, Arizona, and New Mexico. In an area of over $207,200 \mathrm{~km}^{2}$ in these States (Gregory and Moore, 1931, p. 46), it is characterized by conglomerate-filled channels as much as 30 $\mathrm{m}$ deep (McKee, 1954, p. 34; Baker, 1946, p. 57). Elsewhere, the $\operatorname{Tr}-1$ unconformity, at least locally, is even more pronounced; in north-central Utah, $610 \mathrm{~m}$ of Permian rocks are beveled out in a distance of $16 \mathrm{~km}$ (Baker and Williams, 1940 , p. 624). In eastern Utah, the Moenkopi is separated from underlying Permian rocks by a distinctive lithologic break (Schell and Yochelson, 1966, p. D67). Irwin (1971) demonstrated that the unconformity separating Permian and Triassic rocks is readily traced in the subsurface of southern Utah. In contrast, Permian and Triassic rocks are difficult to distinguish in central Wyoming and in adjacent areas to the east and southeast because they are lithologically alike and fossils are sparse or absent. The contact between Permian and Triassic rocks in this region has been interpreted as either representing continuous deposition or a hiatus; available data are equivocal (Sheldon and others, 1967, p. 164-166; Maughan, 1967, p. 145-146). Lacking evidence to the contrary and because most of the other unconformities in overlying Triassic and Jurassic rocks are widespread, we suggest that the $\operatorname{Tr}-1$ unconformity also is present throughout this area.

In parts of Arizona, the Tr-1 unconformity separates Permian rocks of Leonardian age from rocks of middle Early Triassic age (McKee, 1954, p. 34). In most other parts of the Western Interior, the Permian rocks below the Tr-1 unconformity are of Guadalupian age (McKee and others, 1959, pl. 2). Rocks representing the Ochoan Series apparently are absent but the significance of this is uncertain. Inasmuch as the oldest rocks directly above the $\mathrm{Tr}-1$ surface contain a fauna indicating a late Otoceratan age (Kummel, 1954, p. 183, early but not earliest Early Triassic), the time required for uplift and erosion of Permian rocks and the burial of the Tr-1 erosion surface could be as little as 1-2 m.y. (million years) or as much as 5-6 m.y.

\section{TR-2 UNCONFORMITY}

The Tr-2 unconformity is found at the base of the Crow Mountain Sandstone in north-central Wyoming and at the base of the Jelm Formation in Wyoming and north-central Colorado. In the areas of northern Wyoming where the Alcova Limestone is absent, the unconformity is difficult to demonstrate; it is marked only by the change from slopeforming siltstone and very fine grained sandstone in the Red Peak Formation to cliff-forming fine- to medium-grained, 
generally crossbedded sandstone in the overlying Crow Mountain. In central Wyoming, where the Tr-2 unconformity is directly on the Alcova Limestone, it is inconspicuous but widespread. Pebbles derived from the Alcova Limestone commonly occur in the basal few inches of the overlying Jelm or Crow Mountain (Love, Johnson, and others, 1945, locs. 6, 10, 12, 16; Love, Tourtelot, and others, 1947, p. 35, at Mill Creek [W28 of this report]; Woodward, 1957, p. 233; Mapel, 1959, p. 27; Tohill and Picard, 1966, p. 2559; and Pipiringos, 1968, p. D13). In southeastern Wyoming and north-central Colorado, where the Alcova is absent and where the Jelm overlies the Red Peak or Lykins Formation, the erosion surface is characterized by dikelike injections of the overlying unit downward into joints of the underlying unit, as at Bull Mountain [C2] (Pipiringos, 1957, pl. 4B) and Boxelder north [C4] $\left(C-C^{\prime}\right.$, pl. 1 , and Pipiringos and O'Sullivan, 1976).

The Tr- 2 unconformity is truncated by the $\mathrm{J}-1$ unconformity northward; by the J-2 unconformity northeastward, eastward, and southward; by the Tr-3 unconformity near Ditch Creek [C9]; and probably in the subsurface between Bull Lake [W22] and Sheep Creek Gap [U3]. (See $A-A^{\prime}$ and map $B$, pl. 1.) It may be truncated by $\operatorname{Tr}-3$ also to the southwest, but extension of $\mathrm{Tr}-2$ northwestward beyond Red Creek [W18] and Bull Lake [W22] $\left(A-A^{\prime}, E-E^{\prime}\right.$, and map $B$, pl. 1) is uncertain. In central Wyoming, the hiatus at the $\mathrm{Tr}-2$ surface does not seem to be great.

In the area southeast of Red Creek [W18] and Bull Lake [W22], the Red Draw Member of the Jelm and its equivalent, the lower part of the Crow Mountain Sandstone, are confined to a shallow syncline whose axis trends northwest, and if extrapolated would cross the WyomingIdaho border near Garns Mountain [I1]. The unfossiliferous Red Draw Member and equivalent rocks are separated from Lower Triassic rocks by the $\mathrm{Tr}-2$ surface and from the overlying Upper Triassic rocks by the Tr-3 surface. Throughout most of the Western Interior, Upper Triassic rocks rest on Lower Triassic rocks and the intervening Middle Triassic is absent. Only in this area of central Wyoming and adjacent parts of Colorado is the Lower and Upper Triassic separated by a sequence of rocks of unknown age. Consequently, the Red Draw and equivalent rocks may well represent the Middle Triassic.

The Tr-2 unconformity is here correlated with an unconformity recognized by Zieglar (1956, p. 174) in the subsurface of the Williston basin of eastern Montana and western North Dakota at the base of his Saude Formation. The Saude Formation of Zieglar (1956) consists of red beds that contain anhydrite inclusions and well-rounded, frosted sand grains. Equivalent strata in the lower part of the Watrous Formation in Saskatchewan, Canada, similarly contain rounded, frosted quartz grains "commonly found in windblown deposits" (Cumming, 1956, p. 167). On the outcrop at Buffalo Gap in the southern part of the Black
Hills, the uppermost part of the Spearfish Formation (unit 4 of P. J. Lewis and H. D. Hadley, in Reeside and others, 1957, p. 1485-1486) was correlated by Lewis and Hadley both with the Saude Formation and with a unit beneath the Gypsum Spring Formation in the Bighorn Basin of Wyoming. The unit below the Gypsum Spring Formation in the Bighorn Basin is the lower part of the Crow Mountain Sandstone of this report. The lower part of the Crow Mountain along the east flank of the Bighorn Mountains, south of Buffalo, contains sparse spherical, frosted quartz grains and irregular lenses and pods of gypsum (Hose, 1955, p. 51, 52, 107). In southeastern Wyoming the Red Draw Member of the Jelm Formation, an equivalent of the lower part of the Crow Mountain Sandstone, also contains abundant rounded, frosted quartz grains (Pipiringos, 1957, stratigraphic sections, p. 35-63). Its lithology and stratigraphic position above the Tr-2 surface indicates a correlation of the Saude Formation with the lower parts of the Jelm and Crow Mountain Formations.

Zieglar (1956, p. 170) has suggested a Jurassic age for the Saude Formation, but correlation with the lower parts of the Jelm and Crow Mountain Formations indicates that the Saude Formation is Triassic and quite possibly Middle Triassic in age.

\section{TR-3 UNCONFORMITY}

The Tr-3 unconformity is one of the most widespread, conspicuous, and widely recognized unconformities discussed herein. Throughout the Colorado Plateau and eastern Uinta Mountains it separates the Chinle Formation from the underlying Moenkopi Formation (Reeside and others, 1957; Stewart, Poole, and Wilson, 1972a, pl. 3; O'Sullivan and MacLachlan, 1975). In northern Utah it lies between the Gartra Grit (Upper Triassic) and the Mahogany (Lower Triassic) Members of the Ankareh Formation, and farther north, in southeastern Idaho, it separates the Upper Triassic Higham Grit from the underlying Lower Triassic Timothy Sandstone Member of the Thaynes Formation. In southwestern Colorado and adjacent areas in Utah and New Mexico it transgresses older Paleozoic and Precambrian rocks. But in northernmost Colorado and in central and southeastern Wyoming, the Tr-3 surface is cut on the Red Draw Member of the Jelm Formation that is classified by the Survey as Late Triassic but is a unit that may be of Middle Triassic age.

The Tr-3 surface separates the Jelm Formation into an upper member, the Sips Creek, and a lower member, the Red Draw. In the Wind River Basin and to the northwest the Tr-3 surface occurs within the Crow Mountain Sandstone. An unconformity described by Tohill and Picard (1966, p. 2552, fig. 5) along the northern margin of the Wind River Basin is here correlated with the Tr-3 surface. Although not recognized by previous workers, the $\operatorname{Tr}-3$ unconformity is undoubtedly present at the Dallas anticline [W34] and at Red Creek [W 18]. Reexamination of the field notes for these 
two measured sections suggests that the Tr-3 unconformity may occur about $7 \mathrm{~m}$ above the top of the Alcova Limestone at the Dallas anticline, and about $2 \mathrm{~m}$ below the lowest pebble zone in the upper part of the Crow Mountain Sandstone at Red Creek (Pipiringos, 1968, p. D11, fig. 7).

The Tr-3 surface is truncated beneath the J-2 unconformity in the central, northwestern, and southern parts of the region studied. (See $B-B^{\prime}$ and map $B$, pl. 1, and Pipiringos and O'Sullivan, 1976.) The relief on this erosion surface in north-central Arizona is as much as $30 \mathrm{~m}$ within $0.8 \mathrm{~km}$, according to McKee $(1954$, p. 38, fig. 11F) and as much as 53 m near Ferry Swale [A2] according to Phoenix (1963, p. 19, fig. 6). Finch $(1959$, p. 135) cited a channel as much as $38 \mathrm{~m}$ deep at the base of the Chinle Formation in Monument Valley, Ariz., and Davidson (1967, p. 29) reported a channel about $58 \mathrm{~m}$ deep in the Circle Cliffs area about $13 \mathrm{~km}$ west of The Post [U24]. Hansen (1965, p. 69) suggested channels at the base of the Chinle on the order of $27 \mathrm{~m}$ deep near Red Bench [U2] on the north flank of the Uinta Mountains. At most other localities to the north, in northeastern and northern Utah, in Idaho, and adjacent areas, the unconformity is conspicuous, but the erosional relief is small.

Correlations of the Chinle Formation with equivalent rock units are shown on plate 1 . Most of them are well established. The correlation of the Tr-3 surface of the Colorado Plateau, however, with a surface in the middle of the Jelm and the Crow Mountain Formations has not previously been made. The Sips Creek Member of the Jelm is a white or reddish-orange crossbedded sandstone in eastern Wyoming and is a stratigraphic equivalent of the Gartra Member of the Chinle Formation. The Sips Creek also correlates with the unnamed redbed unit of Tohill and Picard (1966, p. 2552, 2553).

The Red Draw Member of the Jelm Formation and the Crow Mountain Sandstone Member of the Chugwater Formation as restricted by Tohill and Picard (1966) might be of Middle Triassic age. If so, the time that elapsed between the uplift, erosion, and the burial of the Tr- 3 surface may not have exceeded one million years.

\section{TRIASSIC-JURASSIC BOUNDARY}

The position of the Triassic-Jurassic boundary is somewhat uncertain. The base of the Jurassic clearly lies stratigraphically above the Chinle and equivalent formations which are of undoubted Triassic age. Over a period of many years the different parts of the Glen Canyon Group have undergone several age reassignments which have fluctuated between the Triassic and Jurassic. In northern Arizona and adjacent parts of Utah the Glen Canyon consists in ascending order of Wingate Sandstone, Moenave and Kayenta Formations, and Navajo Sandstone. Locally, in northeastern Arizona the Wingate Sandstone now comprises two members in ascending order: the Rock Point Member and the Lukachukai Member (Harshbarger and others, 1957, p. 8). Throughout most of the Colorado Plateau the Wingate Sandstone is made up solely of equivalents of the Lukachukai Member. The Rock Point Member of undoubted Late Triassic age is separated from the Lukachukai Member by a widespread unconformity the $\mathrm{J}-0$ surface of this report. Above the J-0 unconformity, the Wingate Sandstone (Lukachukai Member), the partly equivalent Moenave Formation, and overlying parts of the Glen Canyon Group are essentially a single stratigraphic unit that probably was deposited without interruption. Furthermore, most of the Glen Canyon Group, excluding the Rock Point Member, is bounded at the base and top by unconformities. Consequently, we believed that the Glen Canyon Group above the Rock Point Member was either all of Triassic age or all of Jurassic age.

Recently the stratigraphic and paleontological evidence suggested that all the Glen Canyon Group was of Triassic age. Lewis, Irwin, and Wilson (1961) and Galton (1971) reviewed all this evidence bearing on the age of the Glen Canyon and related rocks. It is clear that they would have considered all the Glen Canyon of unquestioned Triassic age if it were not for reported intertonguing between the Glen Canyon Group and overlying rocks of Middle Jurassic age. This apparent intertonguing (Wright and Dickey, 1963; Phoenix, 1963, p. 32) has since been found to occur entirely above the Glen Canyon Group (Fred Peterson, oral commun., 1972). As a result, the arguments in favor of a Jurassic age for the upper part of the Glen Canyon Group based on this intertonguing are no longer valid. Furthermore vertebrate fossils from the upper part of the Navajo Sandstone ( $C-C^{\prime}$ and map $B$, pl. 1) were considered to be of probable Triassic age (Galton, 1971) because of their similarity to vertebrates in the presumed Triassic Newark Group of eastern United States. Consequently a Triassic age for all the Glen Canyon seemed obvious (Galton, 1971, fig. 13, column $\mathrm{H}$ ).

New studies of the Newark Group, however, have now reopened the question of the age of the Glen Canyon Group. Previously all the Newark Group had been assigned a Triassic age (McKee and others, 1959, table 1), but now much of it apparently may be of Jurassic age. Palynological studies by Cornet and Traverse $(1975$, p. 26,29$)$ indicate that the middle part of the Portland Formation in The Hartford basin of Connecticut and Massachusetts is of Early Jurassic age (Pliensbachian Age or younger). Dinosaurs found in the upper part of the Portland Formation might be as young as Early Jurassic (Toarcian Age). Closely related dinosaurs in the upper part of the Navajo Sandstone (Galton, 1971) therefore might indicate a late Early Jurassic age for that part of the Navajo. Moreover, pollen from the Moenave Formation in the lower part of the Glen Canyon Group is also thought to be of Jurassic age (Peterson and others, 1977). This new information suggests that the Glen Canyon Group and equivalent Nugget and Glen Canyon Sandstones 
throughout most of the Colorado Plateau may be middle Early Jurassic in age. In parts of northeastern Arizona and northwestern New Mexico, however, where the Upper Triassic Rock Point Member is present as a lower part of the Wingate Sandstone, the age of the Glen Canyon Group is considered by the U.S. Geological Survey to be of Late Triassic and Early Jurassic. We however believe that the Rock Point Member is separated from the Lukachukai Member by an unconformity and that the age of the Rock Point is not an indication of the age of the overlying Lukachukai. Until all the paleontologic evidence in the Glen Canyon Group is evaluated, the age of the Glen Canyon Group is considered by the Geological Survey to be Triassic and Jurassic. The age of the Nugget Sandstone is considered by the Survey to be Triassic(?) and Jurassic(?).

\section{JURASSIC UNCONFORMITIES}

\section{J-0 UNCONFORMITY}

The $\mathbf{J}-0$ unconformity is at the base of the Glen Canyon Group and the equivalent Nugget Sandstone over wide areas of Nevada, Arizona, New Mexico, Colorado, Utah, Wyoming, and Idaho. The J-0 unconformity in most of this area lies at the top of the Chinle Formation or the equivalent Popo Agie Formation. In northeastern Arizona and adjacent areas it is at the top of division A, which is the uppermost subdivision of the Chinle Formation as originally defined by Gregory (1917, p. 42). The base of divison A is gradational, indicating an affinity with underlying parts of the Chinle Formation. The upper unconformable contact of division A, wherever we examined it, is a distinct even surface marked by sparse coarse grains in basal beds of the Wingate Sandstone and by sandstone wedges extending from the Wingate down into division A (like the one shown in Gilluly and Reeside, 1928, pl. 17A). The unconformity, at places, is particularly noticeable because uppermost beds of division A weather to a recess as much as $1 \mathrm{~m}$ deep and basal beds of the Wingate overhang the recess.

Division A has been given two different names and assigned to two different formations. Harshbarger, Repenning, and Irwin (1957) removed division A from the Chinle Formation, in much of northeastern Arizona, and assigned it as the lower member of the Wingate Sandstone and named it the Rock Point Member. The Rock Point Member supposedly intertongues extensively (Harshbarger and others, 1957, p. 7) with the overlying parts of the Wingate Sandstone, but we have seen no evidence to support this interpretation. In the Monument Valley area of Arizona, at the south end of the Monument upwarp, beds equivalent to division $A$ are assigned to the Church Rock Member of the Chinle; they do not indicate any intertonguing with the overlying Wingate Sandstone (Witkind and Thaden, 1963, p. 34).

The $\mathrm{J}-0$ unconformity is truncated by the $\mathrm{J}-2$ unconformity at the base of the Twin Creek Limestone a short distance to the north of Garns Mountain [I1] in Idaho, and by the J-1 surface at the base of the Gypsum Spring Member of the Twin Creek Limestone in northwestern Wyoming. The $\mathbf{J}-2$ unconformity at the base of the Entrada Sandstone or Canyon Springs Sandstone Member of the Sundance Formation terminates the J-0 unconformity in south-central Wyoming, western Colorado, and northwestern New Mexico ( $B-B^{\prime}, C-C^{\prime}, A-A^{\prime}$, and map $B$, pl. 1).

In southwestern Utah and southern Nevada the J-0 unconformity is an erosion surface that bevels across older parts of the Chinle Formation. Local relief on the unconformity is as much as $3 \mathrm{~m}$. Basal beds of the Glen Canyon Group above the unconformity carry chert, quartz, limestone, and siltstone fragments; they range in size from granules to cobbles and some of them are derived from the Chinle (Wilson and Stewart, 1967, p. D13-D14). In the Circle Cliffs area of south-central Utah the J-0 unconformity is a well-defined horizon with only slight relief. Locally, however, basal beds of the Wingate Sandstone, containing granules and pebbles of gray chert as much as $25 \mathrm{~mm}$ across, fill erosional depressions cut 5-8 $\mathrm{m}$ into the top of the Chinle Formation (Davidson, 1967, p. 36 and 106). In the San Rafael Swell in central Utah, the upper surface of the Chinle Formation is marked by cracks filled to depths of $2-3 \mathrm{~m}$ with sandstone derived from the overlying Wingate Sandstone (Gilluly and Reeside, 1928, p. 68). Along the Grand Hogback in Colorado, on the west side of the White River uplift, the J-0 unconformity of this report is described as even and well defined (Fischer, 1960, p. 9). In the western Uinta Mountains and in northwestern Colorado the Glen Canyon is in sharp contact along the $\mathrm{J}-0$ surface with the upper part of the Chinle and commonly displays chert and other pebbles in its basal few inches. In central Wyoming, the $\mathrm{J}-0$ unconformity is at the base of the Bell Springs Member of the Nugget Sandstone, and the basal few inches of the Nugget is likewise marked by chert and other pebbles. (See Pipiringos, 1968, p. D17; High and Picard, 1965, p. 51, fig. 1, and p. 53-56.)

The length of time required to form the $\mathrm{J}-0$ unconformity depends on the age of the enclosing strata. The youngest stratigraphic unit below the J-0 unconformity is the Chinle Formation of Late Triassic age. In the Uinta Mountains fossil dinosaur footprints from near the top of the Chinle indicate a fauna known elsewhere only from the Lockatong Formation (Donald Baird, written commun., 1964) of Late Triassic (Carnian) age in New Jersey. Consequently we believe the Chinle Formation is mostly of Carnian age; the uppermost part possibly is as young as early Norian. The base of the oldest stratigraphic unit above the unconformity is the Wingate Sandstone (Lukachukai Member) and equivalents. Consequently the formation of the $\mathrm{J}-0$ surface would approximate the duration of the Early Jurassic Hettangian and Late Triassic Norian and Rhaetian Stages or as long as 7-10 m.y. Inasmuch as the $\mathrm{J}-0$ surface is not 
progressively overlapped by younger units it is not possible to estimate a rate of transgression.

\section{J-1 UNCONFORMITY}

The $\mathrm{J}-1$ unconformity is recognized mainly in Wyoming and adjacent parts of Idaho and Utah, where it is the contact between the overlying Gypsum Spring Formation (locally Gypsum Spring Member of the Twin Creek Limestone) and the underlying Nugget Sandstone or older rocks. The J-1 unconformity is here correlated with an erosion surface recognized by Nordquist (1955, fig. 2, p. 104) at the base of the Nesson Formation in the subsurface of the Williston basin, and with the erosion surface at the base of the Temple Cap Sandstone (Peterson and Pipiringos, 1978) of southwestern Utah. In the Williston basin, the J-1 surface is cut on the Saude Formation of Ziegler, 1955; in southwestern Utah it is cut on the Navajo Sandstone. The $\mathrm{J}-1$ surface is limited in all directions by the $\mathrm{J}-2$ unconformity $\left(B-B^{\prime}, C-C^{\prime}, E-E^{\prime}\right.$, and map $\left.B, \mathrm{pl} .1\right)$.

Inasmuch as the Temple Cap Sandstone, the Gypsum Spring and the Nesson Formations overlie the J-1 surface and underlie the $\mathrm{J}-2$ surface, they are here considered nearly exact correlatives. Furthermore, the Dunham Salt of Anderson (1966) is generally considered a facies of the basal member of the Nesson Formation (Nordquist, 1955; Rayl, 1956; Francis, 1957, Sandberg, 1959; and Dow, 1964), and is therefore equivalent to some part of the Gypsum Spring Formation. The Nugget Sandstone is the youngest formation beneath the J-1 surface. The Gypsum Spring Formation, the oldest formation above it, is of earliest Middle Jurassic (Bajocian) age. The elapsed time between uplift and erosion of the Nugget Sandstone and equivalent rocks and deposition of the overlying rocks probably is about 2-3 m.y. depending on the exact age of the enclosing units.

\section{J-2 UNCONFORMITY}

The J-2 unconformity extends throughout the Western Interior. All the older unconformities are truncated by the $\mathrm{J}-2$ surface in one part of the area or another. The areal extent and stratigraphic position of the J-2 surface and its relationship to other surfaces are shown in the five restored sections and the two paleogeologic maps on plate 1. At several localities, younger unconformities truncate and destroy the J-2 surface. The Cretaceous Dakota Sandstone, for example, rests on Precambrian rocks in north-central New Mexico (Muehlberger and others, 1960), and the J-2 surface in this area has been destroyed. In north-central Colorado, near Hot Sulphur Springs [C25], the J-5 surface at the base of the Morrison Formation has eroded the J-2 surface, removing the chert pebbles commonly associated with the J-2 surface. Pebbles found at the base of the Morrison at that locality are mainly quartz derived from the underlying Precambrian. The most conspicuous example of the destruction of the J-2 surface by younger unconformities occurs near ancient Belt Island in western Montana (Cob- ban, 1945; Imlay and others, 1948). The eroded surface of that positive area is bounded by the $\mathrm{Tr}-1$ erosion surface beneath Triassic rocks on its south side, is bounded by the $\mathrm{J}-1$ erosion surface below the Nesson Formation on the east side (map $B$, pl. 1), and is completely girdled by the $\mathrm{J}-2$ surface under the Sawtooth, Piper, and Rierdon Formations (map $A$, pl. 1). (See also $E-E^{\prime}$, pl. 1, near $E^{\prime}$.) The J-4 surface at the base of the Swift has cut through the Rierdon and Sawtooth Formations and has eroded away the preexisting $\mathrm{J}-2$ surface. Most of the pebbles associated with the J-4 surface here are unlike those associated with the J-2 surface at Heath [M4]. The J-4 pebbles have a source to the west. Those at Heath are locally derived. The J-4 surface in turn was breached by the erosion that formed the J-5 surface prior to the deposition of the Morrison Formation. The eroded surface of the ancient Belt Island paleo-high thus comprises parts of the $\operatorname{Tr}-1, \mathrm{~J}-1, \mathrm{~J}-2, \mathrm{~J}-4$, and $\mathrm{J}-5$ surfaces.

The J-2 surface has little relief throughout most of the area. Only about $17 \mathrm{~m}$ of erosional relief in a distance of 16 $\mathrm{km}$ was noted in central Wyoming (Pipiringos, 1968, p. D19). In the subsurface in the southern part of the Powder River Basin, however, Curry and Hegna (1970, fig. 14) showed about $24 \mathrm{~m}$ of relief between two drill holes less than $0.6 \mathrm{~km}$ apart. Locally in Wyoming, near Douglas [W41], the relief is as much as $180 \mathrm{~m}$ on this surface (Dresser, 1959), and in northwestern Montana, in the Sun River Canyon area, about $210 \mathrm{~m}$ is inferred by Mudge (1972, p. A42). About 58 $\mathrm{m}$ of relief in about $11 \mathrm{~km}$ was noted near Kremmling, Colo. [C24] (Pipiringos and others 1969, locs. G and J, fig. 3). Wright and Dickey $(1957$, p. 352) and Pipiringos and O'Sullivan (1975) noted more than $9 \mathrm{~m}$ of relief between the base and the top of a hill of Navajo Sandstone buried by the Entrada on this surface at Sevenmile Canyon [U19] near Moab, Utah. Fred Peterson (oral commun., 1971) found four such buried hills at this unconformity with from 5-12 m of relief in the Kaiparowits region of south-central Utah.

The J-2 surface differs from nearly all the others in that throughout most of its extent it is associated with chert pebbles, shown in all our restored sections by a triangular symbol. The unconformity and(or) the associated pebbles have not been recognized everywhere, but have been described from widely scattered localities. The pebbles have been reported in the subsurface and on the outcrop at the base of the Sawtooth, Piper, Sundance, and Twin Creek Formations in Montana, South Dakota, Wyoming, Idaho, and Utah (Cobban, 1945; Hose, 1955; Imlay, 1956, 1967, Mapel, 1959; Mudge, 1972); at the base of the Sundance in the Wind River Basin of Wyoming (Love, Tourtelot, and others, 1945; Tourtelot, 1953; Woodward, 1957; Horn, 1963); in the Black Hills of South Dakota and Wyoming (Imlay, 1947; Mapel and Bergendahl, 1956; Robinson and others, 1964); in southeastern Wyoming (Dresser, 1959); in central Wyoming (Reynolds, 1968; Merewether, 1972a, b); in southeastern Wyoming and northeastern Colorado 
(Pipiringos and O'Sullivan, 1976); from near the top of the Navajo Sandstone on the south flank of the Uinta Mountains in Utah (Kinney, 1955); and in southeastern Utah (Gilluly and Reeside, 1928; Wright and Dickey, 1957, 1958; Pipiringos and O'Sullivan, 1975); and from the base of the Entrada Sandstone in western Colorado (Cater, 1970; Dyni, 1968; McKay, 1974). An erosion surface (the J-2 unconformity of this report), as well as the associated chert pebbles, has been recognized at the top of the Navajo Sandstone in southern Utah and northern Arizona by Peterson (1973), Peterson and Barnum (1973a, b) and by Zeller and Stephens (1973). The J-2 surface in this area is discussed in more detail by Peterson and Pipiringos (1978). The pebbles have been recognized in northwestern New Mexico by Moench and Schlee (1967, p. 7) and by Green (1974, p. D4). Not generally recognized, heretofore, is that the pebbles reported in all these scattered places overlie a single widespread surface of erosion. Also the chert pebbles that characterize the unconformity are of limited variety that can be identified with certainty as to source. Recognizing and extending this surface into areas where it had not previously been known solves many old correlation problems.

Chert pebbles in general are incorporated in rocks directly on the erosion surface but in a few places they occur within a few centimeters to a meter or two above it. Locally, the pebbles are incorporated in dike-like injections of the pebble-bearing units into the underlying unit. Some of these injections are wedge-shaped and undoubtedly occupy widened joints as at Horse Creek, Wyo. [W61], Four Mile Canyon, Colo. [C27] (Pipiringos and O'Sullivan, 1976, locs. 8,22 ), and Burns, Colo. [C29]. Others occupy irregularly shaped cavities in the underlying unit which were hollowed out by erosion or weathering as at Arches National Monument, Utah [U20] (Pipiringos and O'Sullivan, 1975, fig. 4f).

The chert pebbles are of four principal kinds: (1) pebbles banded in dark and light shades of gray, derived from the Gypsum Spring Formation (see Pipiringos, 1968, fig. 18); (2) pink and red pebbles, most bleached by sunlight to shades of white, pale red, cream, and light gray, and eroded from the Glen Canyon Group, mainly from the Navajo Sandstone (see Baker and others, 1936, pls. 13-14; Pipiringos and O'Sullivan, 1975, fig. 6); (3) dark-red pebbles, some banded yellow, derived from the Petrified Forest Member of the Chinle and its equivalents; and (4) dark-gray and black, a few dark-red, and locally some banded blue and tan chert pebbles derived from Paleozoic rocks. At places in northwestern Colorado near Precambrian terranes, metamorphic rock fragments are found in rocks directly above the J-2 unconformity (Pipiringos and others, 1969, p. N17). Frederickson, De Lay, and Saylor (1956, p. 2132-35, 2141-2144) identified several varieties of pebbles derived locally from Paleozoic rocks that are associated with the J-2 surface in the Canon City area, Colorado.
An estimated 80 percent of the pebbles associated with the unconformity were eroded from the Gypsum Spring Formation and from the Glen Canyon Group. Of the remainder, about 10 percent came from the Chinle Formation, 8 percent from Paleozoic rocks, and 2 percent from Precambrian rocks. The chert pebbles that were derived from the Gypsum Spring Formation came from bedded chert lenses and nodules in marine limestone beds occurring near the top of the formation. The chert pebbles derived from the Glen Canyon Group came principally from cherty limestone lenses that formed in ephemeral lakes among dunes prior to consolidation of the Navajo Sandstone. Similar limestone lenses are present, but scarce, in the Kayenta Formation and in the Wingate Sandstone. Pebbles from the Chinle Formation are derived from bedded chert that formed in lakes that existed during deposition of the Petrified Forest Member and the ocher member of the Chinle Formation. All these cherts are composed almost entirely of fibrous chalcedony, but the different types can be distinguished from each other both in thin section and in hand specimen.

Most pebbles on the J-2 surface that were derived from Paleozoic and Precambrian rocks are nondescript. A possible exception are the unique pebbles found on the east side of the Bighorn Basin. These chert pebbles, probably eroded from Paleozoic rocks, are alternately banded tan and milky blue. Parallel contacts between the thin bands are interrupted where digitations of blue chert fill what seem to be minute shrinkage cracks developed in the tan bands.

Chert pebbles derived from the Gypsum Spring Formation and the Glen Canyon Group are angular to subrounded and most of them are wind polished. So-called "dreikanter," supposedly derived from within and indicative of the environment of deposition of the Navajo Sandstone in the San Rafael Swell and Green River Desert of Utah (Baker and others, 1936, p. 52-53, and pls. 13, 14), instead mark the $\mathrm{J}-2$ unconformity of that area. Less common are wind-polished pebbles derived from the Gypsum Spring Formation and associated with the J-2 surface in the Wind River and Bighorn Basins of Wyoming and in the Pryor Mountains of Montana. Most of these pebbles are thinly banded light to medium gray. Many are angular and are enclosed in a black rind less than $0.5 \mathrm{~mm}$ thick; they obviously were not transported far before the desert varnish was developed. In some places the chert pebbles in rocks directly above the $\mathrm{J}-2$ surface and associated with it can be distinguished from angular fragments of chert beds directly underlying the J-2 surface only by their desert patina or by their high polish.

At many places, as at Dry Muddy Creek [W8] and Douglas, Wyo. [W41], the Gypsum Spring-derived pebbles are well rounded as though worn by wave action on a beach, or more probably by transportation in ancient stream channels; they were later fluted, etched, and polished by the wind. Most of these rounded pebbles are a few centimeters in 
maximum diameter, but locally they are as much as $10-13 \mathrm{~cm}$ long.

Recognition of the $\mathrm{J}-2$ surface modifies or at places strengthens previous stratigraphic interpretations. The Gypsum Spring Formation throughout its extent underlies the $\mathrm{J}-2$ surface; the Piper and Carmel Formations overlie the J-2 surface. Although all these formations are of Middle Jurassic age, the Gypsum Spring Formation cannot be equivalent to any part of the Piper or Carmel Formation. The correlation of the Gypsum Spring Formation in northern Wyoming and southern Montana with the Nesson Formation in the subsurface of Williston basin is obvious, as is correlation of the Piper Formation in its type locality near the town of Piper in central Montana with the Piper in the subsurface of the Williston basin. No attempt has been made in this report to draw a cross section through the Williston basin. Excellent cross sections of the Williston basin were shown by Nordquist (1955), Rayl (1956), Francis (1957), Sandberg (1959), and Dow (1964).

Where similar rocks underlie and overlie the erosion surface, recognition of the $\mathrm{J}-2$ unconformity makes it possible to separate them. Thus, in the east half of the Bighorn Basin as at Sykes Mountain [W2], and in the west half of the Wind River Basin as at Red Creek [W18], near North Fork of Sage Creek [W23], and Peevah Creek [W24], the red beds of the Piper Formation can be separated from similar red beds in the underlying Gypsum Spring Formation. In central Wyoming, at Green Mountain [W44], crossbedded white sandstone of the Canyon Springs Member of the Sundance can be distinguished from the Nugget Sandstone. In the Freezeout Hills [W48, W53, and W54], white crossbedded sandstone in the Canyon Springs can be separated from similar rocks in the underlying Jelm Formation (Pipiringos, 1968, figs. 9, 15, 16). Along the Front Range, as at Owl Canyon [C6] in Colorado, reddish-orange crossbedded Canyon Springs Sandstone Member of the Sundance Formation can readily be distinguished from the reddish-orange crossbedded Jelm Formation. East of the pinchout of the Carmel Formation, in the eastern part of the Uinta Mountains, in western Colorado, in eastern Utah, and in northeastern Arizona, the $\mathrm{J}-2$ unconformity permits the confident separation of the Entrada Sandstone from the underlying Glen Canyon or Navajo Sandstone.

The recognition of the $\mathrm{J}-2$ surface clarifies the relationship of the Navajo Sandstone to some overlying units. Extensive intertonguing has been described in northern Arizona and southern Utah between the underlying Navajo and the overlying Carmel Formation (Wright and Dickey, 1963; Phoenix, 1963, p. 32-33). This reported intertonguing, however, occurs above the Navajo Sandstone entirely within units of Middle Jurassic age. The Temple Cap Member of the Navajo Sandstone has been considered to be a tongue extending into the Carmel Formation. Recent stratigraphic studies by Peterson and Pipiringos (1978), however, show that instead of being a tongue of that formation, the Temple Cap is everywhere separated from the Navajo Sandstone by the J-1 unconformity. Similarly, the Thousand Pockets, heretofore considered to be a tongue of the Navajo Sandstone, instead interfingers with the Carmel Formation as much as $60 \mathrm{~m}$ above the $\mathrm{J}-2$ unconformity and nowhere joins the main body of the Navajo.

The J-2 surface has been traced by us into northwestern New Mexico where its recognition solves a vexing stratigraphic problem. In the Wingate area [NM15], the J-2 surface resıs on the Chinle Formation $\left(C-C^{\prime}, \mathrm{pl} .1\right)$. Many pebbles and granules associated with the $\mathrm{J}-2$ surface are from the Chinle Formation, but about half the granules are types derived from cherty limestone lenses of the Glen Canyon Group. A sequence of partly crossbedded sandstone immediately overlying the J-2 surface was correlated with the Wingate Sandstone by Harshbarger, Repenning, and Irwin $(1957$, p. 8). Inasmuch as this sandstone sequence overlies the J-2 surface, it cannot be an equivalent of the Wingate Sandstone, which is beneath the J-2 surface. The relation of the $J-2$ surface convinces us that the partly crossbedded sandstone sequence is an equivalent of the San Rafael Group and can be correlated with either the Carmel Formation (O'Sullivan and Craig, 1973, p. 79-81) or the Entrada Sandstone. Moench and Schlee $(1967$, p. 6) considered it to be the lower sandstone unit of the Entrada. Green(1974) has recently named this sandstone the Iyanbito Member of the Entrada Sandstone.

The widespread $\mathrm{J}-2$ surface must have formed in a relatively short period of time. The oldest formation directly above the $\mathrm{J}-2$ surface is apparently the Sawtooth Formation in Montana. The lower part of the middle member of this formation contains ammonites of middle Bajocian age equivalent to the Stephanoceras humphresianum ammonite zone (Imlay, 1956, p. 563; 1967, p. 21; 1973, p. 34). The youngest formation directly below the J-2 surface is the Gypsum Spring Formation of central and northern Wyoming. This formation has not yielded ammonites, but its pelecypod and gastropod fauna (Imlay, 1945; Love, Tourtelot, and others, 1945) are probably of middle Bajocian age (R. W. Imlay, oral commun., 1974). These fossils were collected from a limestone bed that is at most no more than about $5 \mathrm{~m}$ below the $\mathrm{J}-2$ surface. Consequently only about 1 million years elapsed between uplift and erosion of the Gypsum Spring and initial deposition of the Sawtooth.

The distribution of the stratigraphic units that directly overlie the $\mathrm{J}-2$ surface (map $A, \mathrm{pl}$. 1) indicates that the $\mathrm{J}-2$ surface was overlapped from west to east and from north to south. Initially, the J-2 surface was buried in the western part of the area by the Carmel Formation and Twin Creek Limestone and in the Williston basin by the Piper Formation. The youngest unit directly above the $\mathrm{J}-2$ surface is the Canyon Springs Member of the Sundance Formation of 
north-central Colorado (Pipiringos and others, 1969), which is now considered to be of early to middle Callovian age ( $R$. W. Imlay, written commun., 1974). Estimates made from the Phanerozoic time scale (Geol. Soc. London, 1964, as modified by van Hinte, 1976) indicate that deposition on the $\mathrm{J}-2$ surface began in the Williston basin about $161 \mathrm{~m} . \mathrm{y}$. ago and ended in central Colorado about 156 m.y. ago. About 5 m.y. was required for progressive overlapping of an area measuring about $500 \mathrm{~km}$ east-west and about $800 \mathrm{~km}$ north-south. This is an average rate of advance of the transgressing seas of about $100-160 \mathrm{~km}$ per $1 \mathrm{~m} . \mathrm{y}$.

Deposition of the rock sequence between the J-2 and J-3 unconformities was continuous for the period of $17 \mathrm{~m} . \mathrm{y}$., which is approximately the length of time it took to deposit the Twin Creek Limestone and the Preuss Sandstone in southeastern Idaho and the Piper and Rierdon Formations in the Williston basin. According to Imlay (1967, p. 4; 1952b, p. 1739), the Twin Creek is as much as $870 \mathrm{~m}$ thick in the vicinity of Salt Lake City, Utah, near Devils Slide [U1], and the Preuss is as much as $400 \mathrm{~m}$ thick at Thomas Fork Canyon, Idaho, near Preuss Creek [14], for a combined thickness of about $1270 \mathrm{~m}$. The rate of deposition or rate of hingeline subsidence was about $0.07 \mathrm{~mm}$ per year. By contrast, the combined thickness of the Piper and Rierdon Formations in the Williston basin is about $180 \mathrm{~m}$, according to Francis (1957, figs. 9, 10). In that basin the rate of deposition or basin subsidence was much less - about 0.01 mm per year.

\section{J-3 UNCONFORMITY}

The J-3 unconformity underlies the Curtis Formation in the San Rafael Swell of central Utah and in the Uinta Mountains of northeastern Utah and northwestern Colorado. Chert pebbles occur on, or not far above, the J-3 surface throughout much of its areal extent. The chert pebbles are black and white and well rounded; they are unlike the pebbles associated with the J-2 surface. The pebbles on the J-3 surface seem to have a source somewhere to the west of the San Rafael Swell. They are largest and most numerous in the Curtis on the north side of Salina Canyon, about $6 \mathrm{~km}$ southeast of Salina, Utah, and smallest and least numerous in channel-like remnants of the Curtis in the vicinity of Manila, Utah, near Red Bench [U2] on the north flank of the Uinta Mountains. The northeastward decrease in pebble size indicates a corresponding direction of transport; moreover, crossbedding studies show that the Curtis Formation was deposited by northeast-flowing currents (Dickey and Wright, 1958, p. 59). Whether the J-3 surface extends farther to the northwest and northeast than presently recognized is uncertain. Locally, the basal contact of the Pine Butte Member of the Sundance Formation, which is at the stratigraphic level of the J-3 surface, is a well-defined surface, but at places the Pine Butte Member seems to intertongue with the underlying Lak Member of the Sundance. An unconformity (the J-3 surface of this report) has been identified in southern Utah and northern Arizona where it separates the sandstone at Romana Mesa from the Entrada (Peterson, 1973; Peterson and Barnum, 1973a, b). Whether the J-3 surface can be identified in southwestern Colorado or northwestern New Mexico is uncertain. The limits of the J-3 surface have been established only on the north and south sides of the eastern part of the Uinta Mountains, where it is truncated by the J-4 surface, and along the north side of White Mesa in northeastern Arizona where it is truncated by a Cretaceous erosional surface. Its disappearance by truncation beneath the J-5 surface toward the southwest ( $E-E^{\prime}$, fig. 2) near Black Ridge [U21] is inferred, but seems probable.

Topographic relief on the J-3 surface has not been systematically investigated. A cursory examination of the J-3 surface at the contact of the Curtis Formation and Entrada Sandstone in the northeastern part of the San Rafael Swell showed an inferred relief of as much as $14 \mathrm{~m}$ in about $3 \mathrm{~km}$.

The J-3 unconformity may be more widespread than presently recognized. However, unless additional work establishes the existence of the $\mathrm{J}-3$ surface in other parts of the Western Interior, it appears that its utility for correlation will be limited to Utah, northern Arizona, and possibly westernmost Colorado.

The youngest stratigraphic unit beneath the J-3 surface is the Entrada Sandstone. The oldest unit above the surface is the Curtis Formation, which probably correlates with the Pine Butte Member of the Sundance Formation. The part of the Entrada directly beneath the J-3 surface near Red Bench [U2], Utah, seems to correlate with the Lak Member of the Sundance. The J-3 surface, therefore, is bracketed within beds of middle Callovian age. The middle Callovian lasted about 2 m.y., according to the Phanerozoic time scale (Geol. Soc. London, 1964, as modified by van Hinte, 1976). The elapsed time between uplift and erosion of the Entrada and the onset of burial beneath the Curtis, therefore, is estimated to be less than $1 \mathrm{~m} . \mathrm{y}$.

\section{J-4 UNCONFORMITY}

The J-4 unconformity is a surface of erosion that underlies the Redwater Shale Member of the Sundance in South Dakota, Wyoming, and adjacent parts of northeastern Utah and northwestern Colorado. It also is present at the base of the Swift Formation in Montana. Throughout most of this area, it is marked only by a sharp change in lithology and an abrupt appearance of belemnites above the unconformity, but locally, truncation of the units beneath the J-4 surface is pronounced. The truncation has been described in Montana around the site of ancient Belt Island, where both the underlying Rierdon and the Sawtooth Formations are truncated and the Swift rests on Paleozoic rocks (Cobban, 1945, fig 6, $C-C^{\prime}$; Imlay and others, 1948, cross sections $\left.A-A^{\prime}, B-B^{\prime}\right)$. Less well known is the complete truncation of the Curtis Formation beneath the $\mathrm{J}-4$ surface within a fairly 
narrow zone that trends northwest along both flanks of the eastern end of the Uinta Mountains. Within this area of northeastern Utah and northwestern Colorado near and including Red Bench [U2], Irish Lake [C3], Deerlodge Park [C16], Calico Draw [C17], and Uranium Peak [C21], the Redwater Shale Member of the Sundance Formation rests on the Entrada Sandstone. A paleogeologic map of the rocks beneath the $\mathrm{J}-4$ surface in the area just described would show a narrow northwest-trending lobe of Entrada Sandstone surrounded by the Curtis Formation. The Redwater Shale Member of the Sundance has been removed by erosion associated with the J-5 surface south of the Uinta Basin in Utah and south and east of northwest Colorado (pl. 1, E-E', B-B'). In southeastern Wyoming the J-4 surface and the overlying Redwater are truncated by the J-5 surface along a northeast-trending line that extends from just north of Red Mountain [W65] to just north of Farthing [W58]. An extrapolation of this trend into the subsurface suggests that the Redwater zero line would intersect the northwest corner of Nebraska and join the zero line of the Redwater shown by Peterson (1972, fig. 7) in South and North Dakota. In map $A$ (pl. 1), areas, such as ancient Belt Island, the windows in the Entrada around Hot Sulphur Springs [C25], south of Red Hill Gap [C37], and north of Ghost Ranch [NM6], are not in color because the J-2 surface in those areas has been destroyed by erosion associated with either the J-5 surface or the period of erosion that preceded the deposition of the Cretaceous Dakota Sandstone.

The topographic relief on the J-4 surface is slight and is not conspicuous locally. Even in the vicinity of ancient Belt Island where hundreds of feet of the underlying stratigraphic units have been cut out, the $J-4$ surface itself on which the Swift was laid down shows little if any topographic relief.

The recognition of the $\mathrm{J}-4$ surface revises some previously accepted correlations. The Curtis Formation as presently recognized by us in the Uinta Mountains consists of two parts that are separated by the $\mathrm{J}-4$ unconformity. The upper part is unmistakably equivalent to the Redwater Shale Member of the Sundance because of its fossil content, its characteristic lithologies and its position above the J-4 unconformity. The lower part of the Curtis beneath the J-4 unconformity is only locally present; it correlates with the type Curtis in the San Rafael Swell. The Curtis Formation intertongues with the overlying Summerville Formation in the San Rafael Swell, and for this reason the two formations are considered partly contemporaneous. Northward from the swell the Summerville is undoubtedly truncated by the $\mathrm{J}-4$ unconformity in the subsurface of the Uinta Basin south of White Rocks Canyon [U5] and near Cliff Ridge [U9], probably not very far north of the axis of the Uncompahgre uplift. In the Uinta Mountains the Summerville is absent and the $\mathrm{J}-4$ surface overlies locally thin remnants of the Curtis Formation. Diagnostic fossils including ammonites of the Cardioceras cordiforme faunal zone, belemnites, and certain pelecypods have been collected from the Redwater Shale Member in the Uinta Mountains. These diagnostic fossils heretofore have been used erroneously to date the age of the Curtis and consequently the Summerville Formation in the San Rafael Swell. Fossils are present in the type Curtis but do not include ammonites and are otherwise largely nondiagnostic. The age of the Curtis and the Summerville in the San Rafael Swell cannot be determined with confidence by the fossils present. The Curtis, in the Uinta Mountains, unconformably overlies the Entrada of early to middle Callovian age and is unconformably overlain by the Redwater of early to middle Oxfordian age. These relationships indicate a late middle or early late Callovian age for the Curtis and for the overlying Summerville, but preclude an Oxfordian Age.

The stratigraphy of the Stump Sandstone has heretofore been poorly understood. In 1964, R. W. Imlay and G. N. Pipiringos examined, very briefly, a few outcrops of the Stump Sandstone in southeastern Idaho and adjacent parts of Wyoming and Utah. At these few localities the Stump lithologically resembled only the Curtis Formation in Utah and a unit in south-central Wyoming now named the Pine Butte Member of the Sundance Formation. The Redwater Shale Member of the Sundance was not recognized in the Stump, although belemnites and Camptonectes bellistriatus Meek indicative of the Redwater Shale Member had been previously reported from the Stump (Mansfield, 1927, p. 101).

By 1974, however, various U.S. Geological Survey field parties had collected additional belemnites and the Oxfordian ammonites Cardioceras? and Cardioceras from the Stump at several localities (R. W. Imlay, 1974, written commun.). Inasmuch as some of these fossils reportedly were collected from near the base of the Stump, it seemed likely that perhaps all of the Stump might be a Redwater equivalent. In order to resolve these uncertainties $R$. W. Imlay and G. N. Pipiringos, in September of 1975, made a more widespread examination of outcrops in southeastern Idaho and western Wyoming. They found that the Stump Sandstone throughout much of the area consists of two parts separated by the $\mathrm{J}-4$ unconformity. The upper part contains belemnites and Cardioceras and is indeed an equivalent of the Redwater Shale Member of the Sundance Formation. At Stump Peak, the type locality, only the lower part of the Stump is present; the upper part was removed prior to the deposition of the Ephraim Conglomerate of Cretaceous age. These relations are shown near $A$ of $A-A^{\prime}$, figure 2, between Stump Creek [I3] and Green River Lakes [W21].

The Summerville Formation is the youngest stratigraphic unit beneath the $\mathrm{J}-4$ surface, and the lowermost part of the Swift Formation is the oldest unit above the $\mathrm{J}-4$ surface. Inasmuch as the Summerville is probably of early late Callovian age and the basal Swift of central Montana contains fossils of latest Callovian age (Imlay, 1947, p. 260, 261, tables I, III), the J-4 unconformity apparently 
developed during a small part of late Callovian time, which lasted about $2 \mathrm{~m}$.y. The time, therefore, that elapsed between uplift and erosion of the Summerville and equivalents and the beginning of deposition of the Swift in central Montana can reasonably be placed at about $1 \mathrm{~m} . \mathrm{y}$.

\section{J-5 UNCONFORMITY}

The J-5 unconformity occurs throughout most of the Western Interior at the base of the Morrison Formation or of the Windy Hill Sandstone Member of the Sundance Formation. The extension of the J-5 surface northward into Montana, northwestern Wyoming, and Idaho, is uncertain. The J-5 surface at the base of the Windy Hill Sandstone Member truncates underlying units in Wyoming and northern Colorado (pl. 1, D- $D^{\prime}$ ). In central Wyoming, the Redwater Shale Member of the Sundance Formation below the Windy Hill consists of four distinct lithologic units. These four units are progressively beveled out by the J-5 surface eastward, southeastward, and southward (Pipiringos, 1957, pl. 5; Pipiringos, 1968, p. D4, fig. 3; Pipiringos, 1972, p. 22, fig. 4; Pipiringos and O'Sullivan, 1976). The Windy Hill is present within an area that is elongate to the northeast and that is about $320 \mathrm{~km}$ wide. An approximate midline of this area extends from about the middle of the Black Hills southwestward to Cross Mountain [C12] and from there southwestward to a point near Escalante [U26] in southwestern Utah. The J-5 surface at the base of the Morrison Formation has been recognized in southwestern Utah as a result of detailed investigations by Fred Peterson and H. D. Zeller (Fred Peterson, oral commun., 1970). The J-5 surface has subsequently been traced from Escalante to the Page area [A3] of northern Arizona, where it separates the sandstone at Romana Mesa from the overlying Morrison Formation (Peterson, 1973; Peterson and Barnum, 1973a, b). Farther west, in southwestern Utah, the J-5 surface is truncated by an unconformity at the base of Cretaceous rocks (pl. 1, C-C', $\left.E-E^{\prime}\right)$.

Opinions are divided as to whether there is an erosional surface between the Swift and the Morrison Formations in Montana. R. W. Imlay (written commun., 1974) states, "No physical evidence for an unconformity between the Swift and Morrison Formations has been found in Montana."We agree that at any one place there is little, if any, physical evidence for the existence of the J-5 surface. However, in southern Wyoming and northern Colorado, for example, the presence of the J-5 surface can be inferred by the regional truncation of the Redwater Shale and Pine Butte Members of the Sundance Formation. (See pl. 1, D- $D^{\prime}$; Pipiringos, 1968, fig. 3 and p. D24; Pipiringos, 1972, fig. 4 and p. 28; Pipiringos, Hail, and Izett, 1969, fig. 3 and p. N16; Pipiringos and O'Sullivan, 1976). Consequently, it seems reasonable to us that some of the variation in thickness of the Swift where overlain by the Morrison in Montana from 1 to $74 \mathrm{~m}$ (pl. 1, $E-E^{\prime}$ near $E^{\prime}$; and Imlay and others, 1948) is attributable to truncation of the Swift by pre-Morrison erosion. At least two other authors are also of the opinion that the contact of the Swift and Morrison Formations in Montana is an erosional surface (Carlson, 1968, p. 1979; Christopher, 1974, p. 6, 43, and 49).

The topographic relief on the J-5 surface is slight. Throughout the areal extent of the Windy Hill Sandstone Member the J-5 unconformity is a nearly flat even surface; a surface almost unmarked by channels even where it bevels underlying units. Apparently, after uplift and erosion of the underlying Redwater Shale Member, the area was reduced to a smooth peneplain. Subsequently the area was invaded by the shallow short-lived Windy Hill sea and the area was then covered by the terrestrial deposits of the Morrison Formation. Where the Windy Hill is absent the J-5 surface is at the base of the Morrison Formation. In many areas the Morrison Formation rests on rocks of different lithology and the base is an erosional surface; in other areas, the Morrison rests on rocks of similar lithology and the contact is poorly defined (Cadigan, 1967, p. 8). The poorly defined contact might be explained as the result of reworking of underlying units by Morrison streams. The J-5 surface at the base of the Morrison is recognized in southwestern Utah (Peterson and Barnum, 1973a, b; Zeller and Stephens, 1973), and in the vicinity of Escalante [U26] the relief on this surface is about $46 \mathrm{~m}$ in a distance of $6 \mathrm{~km}$ (Fred Peterson, oral commun., 1974).

Recognition of the $\mathbf{J}-5$ and the $J-2$ surfaces clarifies some stratigraphic details of the Ralston Creek Formation and the Windy Hill Sandstone Member of the Sundance Formation. The Ralston Creek Formation is described as resting on the Lykins Formation (LeRoy, 1946, fig. 11, p. 51-53). The Ralston Creek Formation, at Ralston Reservoir (the type section), Colo., includes at the base a pebbly sandstone about $5 \mathrm{~m}$ thick that is in contact with the underlying Lykins along the J-2 surface. The pebbly sandstone, in turn, is in contact with overlying strata of the Ralston Creek Formation along the J-5 surface. The basal pebbly sandstone clearly is a continuation of the Canyon Springs Sandstone Member of the Sundance that is present to the north and is entirely older than the Oxfordian Redwater Shale Member of the Sundance (pl. 1, D- $D^{\prime}$, Pipiringos and O'Sullivan, 1976). The upper nonconglomeratic part of the Ralston Creek at Ralston Reservoir [C32] is equivalent to the Morrison to the north. The base of the Ralston Creek Formation in the Canon City embayment of Colorado is marked by abundant wind-polished chert pebbles (Frederickson and others, 1956, p. 2134, fig. 5) of the type that is diagnostic of the $\mathrm{J}-2$ surface along the Front Range of Colorado between Owl Canyon [C6] and Four Mile Canyon [C27]. In the Canon City area the Ralston Creek-Morrison contact is disconformable (Frederickson and others, 1956, p. 2138). These relationships and the $\mathrm{J}-2$ chert pebbles suggest that the Ralston Creek at Canon City is in part, and may be 
entirely, an equivalent of the Canyon Springs in north-central Colorado and of the Entrada Sandstone in northwestern Colorado. The J-5 surface also underlies the Windy Hill Sandstone Member of the Sundance Formation. The arbitrary assignment of the Windy Hill to the Sundance (Pipiringos, 1968, p. D24) obscures its relations to overlying and underlying formations. The Windy Hill regionally truncates all but the basal part of the Sundance Formation. The Windy Hill Sandstone Member, however, grades upward into, and intertongues laterally with, the Morrison Formation.

In eastern and southern Wyoming and adjacent parts of Colorado the uppermost dated beds of the Redwater Shale Member are of middle Oxfordian age and are overlain by the Windy Hill Sandstone Member. The Windy Hill is probably of Kimmeridgian Age inasmuch as it interfingers with the lowermost part of the Morrison Formation, which most previous workers have found to be of Kimmeridgian Age, or younger (Baker, Dane, and Reeside, 1936, p. 58-63; Reeside, 1952, p. 25; Imlay, 1952a, p. 958; Peck, 1957, p. 7). The time required to form the J-5 surface in Wyoming, therefore, is equal to some part of the last third of the Oxfordian. Inasmuch as the duration of the late Oxfordian is about 3. m.y., the hiatus represented by the J-5 surface is probably less than 2. m.y. In northwestern Montana the hiatus, if present, must represent an even shorter time interval because the Swift Formation of that area is of late Oxfordian age (Mudge, 1972, p. A48).

\section{CRETACEOUS UNCONFORMITY}

\section{K UNCONFORMITY}

Cretaceous rocks apparently truncate the Morrison Formation and equivalents everywhere in the Western Interior (Cobban and Reeside, 1952; McGookey and others, 1972). One or more Cretaceous unconformities may be represented in the break between Jurassic and Cretaceous rocks shown in the Correlation of units (pl. 1), but they were not studied by us. The youngest rocks beneath the $\mathrm{K}$ unconformity probably are of latest late Jurassic age (R. W. Imlay, written commun., 1976). The oldest formation above the unconformity - the Ephraim Conglomerate - apparently is of earliest Cretaceous age. The absent latest Jurassic interval probably spans a period of about $3 \mathrm{~m} . \mathrm{y}$.

\section{REFERENCES CITED}

Anderson, S. B., 1966, A look at the petroleum potential of southwestern North Dakota: North Dakota Geol. Survey Rept. Inv. 42.

Baker, A. A., 1946, Geology of the Green River Desert-Cataract Canyon region, Emery, Wayne, and Garfield Counties, Utah: U.S. Geol. Survey Bull. 951, 122 p.

Baker, A. A., Dane, C. H., and Reeside, J. B., Jr., 1936, Correlation of the Jurassic formations of parts of Utah, Arizona, New Mexico, and Colorado: U.S. Geol. Survey Prof. Paper 183, 66 p.
Baker, A. A., and Williams, J. S., 1940, Permian in parts of Rocky Mountain and Colorado Plateau regions: Am. Assoc. Petroleum Geologists Bull., v. 24, no. 4, p. 617-635.

Braddock, W. A., 1963, Geology of the Jewel Cave SW quadrangle, Custer County, South Dakota: U.S. Geol. Survey Bull. 1063-G, p. 217-268.

Brady, L. F., 1936, A note concerning the fragmentary remains of a small theropod recovered from the Navajo Sandstone in Northern Arizona: Am. Jour. Sci., ser.5, v.31, p.150.

Cadigan, R. A., 1967, Petrology of the Morrison Formation in the Colorado Plateau region: U.S. Geol. Survey Prof. Paper 556, 113 p.

Carlson, C. E., 1968, Triassic-Jurassic of Alberta, Saskatchewan, Manitoba, Montana, and North Dakota, in Rocky Mountains Breaking barrier boundaries: Am. Assoc. Petroleum Geologists Bull., v. 52, no. 10, p. 1969-1983.

Cashion, W. B., 1967, Carmel Formation of the Zion Park region, southwestern Utah - A review: U.S. Geol. Survey Bull. 1244-J, p. J1-J9.

Cater, F. W., 1970, Geology of the salt anticline region in southwestern Colorado, with a section on Stratigraphy, by F. W. Cater and L. C. Craig: U.S. Geol. Survey Prof. Paper 637, 80 p. [1971].

Christopher, J. E., 1974, The Upper Jurassic Vanguard and Lower Cretaceous Mannville Groups of southwestern Saskatchewan: Saskatchewan Dept. Mineral Resources Rept. 151, 349 p.

Cobban, W. A., 1945, Marine Jurassic formations of Sweetgrass arch, Montana: Am. Assoc. Petroleum Geologists Bull., v. 29, no. 9, p. 1262-1303.

Cobban, W. A., and Reeside, J. B., Jr., 1952, Correlation of the Cretaceous formations of the Western Interior of the United States: Geol. Soc. America Bull., v. 63, p. 1011-1043.

Cornet, Bruce, and Traverse, Alfred, 1975, Palynological contributions to the chronology and stratigraphy of the Hartford basin in Connecticut and Massachusetts: Geosci. Man, v. 11 (Am. Assoc. Stratig. Palynology, 6th ann. Mtg. Proc., p. 1-33.)

Cumming, A. D., 1956, The Watrous strata in Saskatchewan, in North Dakota Geol. Soc., Williston Basin Symposium, 1st Internat., Bismarck, p. 165-169.

Curry, W. H., and Hegna, E. T., 1970, Stratigraphic oil accumulations in the Lower Sundance Canyon Springs sandstones of Wyoming, in Wyoming Geol. Assoc. 22d Field Conf. Guidebook, Symposium on Wyoming sandstones: p. 51-74.

Dane, C. H., 1935, Geology of the Salt Valley anticline and adjacent areas, Grand County, Utah: U.S. Geol. Survey Bull. 863, 184 p. [1936].

Davidson, E. S., 1967, Geology of the Circle Cliffs area, Garfield and Kane Counties, Utah: U.S. Geol. Survey Bull. 1229, 140 p.

Dickey, D. D., and Wright, J. C., 1958, San Rafael (Entrada) studies [Utah], in Geologic investigations of radioactive deposits - semiannual progress report, June 1 to November 30, 1958: U.S. Geol. Survey TEI-750, p. 59-65, issued by U.S. Atomic Energy Comm. Tech. Inf. Service, Oak Ridge, Tenn.

Dow, W. G., 1964, The Spearfish Formation in western North Dakota, in Williston basin Symposium, 3d Internat., Regina, Saskatchewan, 1964, Proc.: Billings, Mont., Billings Geol. Soc., p. 127-131.

Dresser, H. W., 1959, A field study of the Jurassic "Lower Sundance" beds in southeastern Wyoming: Wyoming Univ. Ph. D. thesis, v. 1, p. 1-112; v. 2, p. 113-667.

Dyni, J. R., 1968, Geologic map of the Elk Springs quadrangle, Moffat County, Colorado: U.S. Geol. Survey Geol. Quad. Map GQ-702.

Ekren, E. B., and Houser, F. N., 1965, Geology and petrology of the Ute Mountains area, Colorado: U.S. Geol. Survey Prof. Paper 481, 74 p. Finch, W. I., 1959, Geology of uranium deposits in Triassic rocks of the Colorado Plateau region: U.S. Geol. Survey Bull. 1074-D, p. 125-164.

Fischer, R. P., 1960, Vanadium-uranium deposits of the Rifle Creek area, Garfield County, Colorado, with a section on Mineralogy, by Theodore Botinelly: U.S. Geol. Survey Bull. 1101, 52 p. [1961].

Francis, D. R., 1957, Jurassic stratigraphy of Williston Basin area: Am. Assoc. Petroleum Geologists Bull., v. 41, no. 3, p. 367-398. 
Fraser G. D., Waldrop, H. A., and Hyden, H. J., 1969, Geology of the Gardiner area, Park County, Montana: U.S. Geol. Survey Bull. 1277, $118 \mathrm{p}$.

Frederickson, E. A., De Lay, J. M., and Saylor, W. W., 1956, Ralston Formation of Canon City embayment, Colorado: Am. Assoc. Petroleum Geologists Bull., v. 40, no. 9, p. 2120-2148.

Galton, P. M., 1971, The prosauropod dinosaur Ammosaurus, the crocodile Protosuchus, and their bearing on the age of the Navajo Sandstone of northeastern Arizona: Jour. Paleontology, v. 45, no. 5, p. 781-795.

Geological Society of London, 1964, Summary of the Phanerozoic time scale: Geol. Soc. London Quart. Jour., v. 120 S, p. 260-262.

Gilluly, James, and Reeside, J. B., Jr., 1928, Sedimentary rocks of the San Rafael Swell and some adjacent areas in eastern Utah: U.S. Geol. Survey Prof. Paper 150-D, p. 61-110.

Green, M. W., 1974, The Iyanbito Member (a new stratigraphic unit) of the Jurassic Entrada Sandstone, Gallup-Grants area, New Mexico: U.S. Geol. Survey Bull. 1395-D, p. D1-D12.

Gregory, H. E., 1917, Geology of the Navajo country; a reconnaissance of parts of Arizona, New Mexico, and Utah: U.S. Geol. Survey Prof. Paper 93, $161 \mathrm{p}$.

Gregory, H. E., and Moore, R. C., 1931, The Kaiparowits region, a geographic and geologic reconnaissance of parts of Utah and Arizona: U.S. Geol. Survey Prof. Paper 164, 161 p.

Hansen, W. R., 1965, Geology of the Flaming Gorge area, Utah-Colorado-Wyoming: U.S. Geol. Survey Prof. Paper 490, 196 p.

Harshbarger, J. W., Repenning, C. A., and Irwin, J. H., 1957, Stratigraphy of the uppermost Triassic and the Jurassic rocks of the Navajo country [Colorado Plateau]: U.S. Geol. Survey Prof. Paper 291, 74 p.

High, L. R., Jr., and Picard, M. D., 1965, Sedimentary petrology and origin of analcime-rich Popo Agie Member, Chugwater (Triassic) Formation, west-central Wyoming: Jour. Sed. Petrology, v. 35, no. 1, p. 49-70.

Horn, G. H., 1963, Geology of the East Thermopolis area, Hot Springs and Washakie Counties, Wyoming: U.S. Geol. Survey Oil and Gas Inv. Map OM-213.

Hose, R. K., 1955, Geology of the Crazy Woman Creek area, Johnson County, Wyoming: U.S. Geol. Survey Bull. 1027-B, p. 33-118 [1956].

Imlay, R. W., 1945, Occurrence of Middle Jurassic rocks in western interior of United States: Am. Assoc. Petroleum Geologists Bull., v. 29, no. 7, p. 1019-1027.

_1947, Marine Jurassic of Black Hills area, South Dakota and Wyoming: Am. Assoc. Petroleum Geologists Bull., v. 31, no. 2, p. 227-273.

1952a, Correlation of the Jurassic formations of North America, exclusive of Canada: Geol. Soc. America Bull., v. 63, no. 9, p. 953-992.

1952b, Marine origin of the Preuss sandstone of Idaho, Wyoming, and Utah: Am. Assoc. Petroleum Geologists Bull., v. 36, no. 9, p. 1735-1753.

1956, Marine Jurassic exposed in Bighorn Basin, Pryor Mountains and northern Bighorn Mountains, Wyoming and Montana: Am. Assoc. Petroleum Geologists Bull., v. 40, p. 562-599.

1964, Marine Jurassic pelecypods from central and southern Utah: U.S. Geol. Survey Prof. Paper 483-C, p. C1-C42.

1967, Twin Creek Limestone (Jurassic) in the western interior of the United States: U.S. Geol. Survey Prof. Paper 540, 105 p.

1968, Lower Jurassic (Pliensbachian and Toarcian) ammonites from eastern Oregon and California: U.S. Geol. Survey Prof. Paper 593-C, p. C1-C51.

1973, Middle Jurassic (Bajocian) ammonites from eastern Oregon: U.S. Geol. Survey Prof. Paper 756, 100 p.

Imlay, R. W., Gardner, L. S., Rogers, C. P., Jr., and Hadley, H. D., 1948, Marine Jurassic formations of Montana: U.S. Geol. Survey Oil and Gas Inv. Prelim. Chart 32.

Irwin, C. D., 1971, Stratigraphic analysis of upper Permian and lower Triassic strata in southern Utah: Am. Assoc. Petroleum Geologists Bull., v. 55 , no. 11, p. 1976-2007.
Izett, G. A., 1968, Geology of the Hot Sulphur Springs quadrangle, Grand County, Colorado: U.S. Geol. Survey Prof. Paper 586, 79 p.

Johnson, R. B., and Baltz, E. H., Jr., 1960, Probable Triassic rocks along eastern front of Sangre de Cristo Mountains, south-central Colorado: Am. Assoc. Petroleum Geologists Bull., v. 44, no. 12, p. 1895-1902.

Kinney, D. M., 1955, Geology of the Uinta River-Brush Creek area, Duchesne and Uintah Counties, Utah: U.S. Geol. Survey Bull. 1007, 185 p.

Kummel, Bernard, 1954, Triassic stratigraphy of southeastern Idaho and adjacent areas [Wyo.-Mont]: U.S. Geol. Survey Prof. Paper 254-H, p. 165-194.

LeRoy, L. W., 1946, Stratigraphy of the Golden-Morrison area, Jefferson County, Colorado: Colorado School Mines Quart., v. 41, no. 2, 115 p.

Lewis, G. E., Irwin, J. H., and Wilson, R. F., 1961, Age of the Glen Canyon Group (Triassic and Jurassic) on the Colorado Plateau: Geol. Soc. America Bull., v. 72, no. 9, p. 1437-1440.

Love, J. D., 1939, Geology along the southern margin of the Absaroka Range, Wyoming: Geol. Soc. America Spec. Paper 20, 134 p.

Love, J. D., Johnson, C. O., Nace, H. L., Sharkey, H. H. R., Thompson, R. M., Tourtelot, H. A., and Zapp, A. D., 1945, Stratigraphic sections and thickness maps of Triassic rocks in central Wyoming: U.S. Geol. Survey Oil and Gas Inv. Prelim. Chart 17.

Love, J. D., Tourtelot, H. A., Johnson, C. O., Sharkey, H. H. R., Thompson, R. M., and Zapp, A. D., 1945, Stratigraphic sections and thickness maps of Jurassic rocks in central Wyoming: U.S. Geol. Survey Oil and Gas Inv. Prelim. Chart 14.

Love, J. D., Tourtelot, H. A., Johnson, C. O., Thompson, R. M., Sharkey, H. H. R., and Zapp, A. D., 1947, Stratigraphic sections of Mesozoic rocks in central Wyoming: Wyoming Geol. Survey Bull. 38, 59 p.

McGookey, D. P., Haun, J. D., Hale, L. A., Goodell, H. G., McCubbin, D. G., Weimer, R. J., and Wulf, G. R., 1972, Cretaceous System, in Geologic atlas of the Rocky Mountain Region, United States of America: Rocky Mtn. Assoc. Geologists, Denver, Colorado, p. 190-228.

MacLachlan, M. E., 1972, Triassic System, in Geologic atlas of the Rocky Mountain Region, United States of America: Rocky Mtn. Assoc. Geologists, Denver, Colorado, p. 166-176.

McKay, E. J., 1974, Geologic map of the Lone Mountain quadrangle, Moffat County, Colorado: U.S. Geol. Survey Geol. Quad. Map GQ-1144.

McKee, E. D., 1954, Stratigraphy and history of the Moenkopi formation of Triassic age: Geol. Soc. America Mem. 61, 133 p.

McKee, E. D., Oriel, S. S., Swanson, V. E., MacLachlan, M. E., MacLachlan, J. C., Ketner, K. B., Goldsmith, J. W., Bell, R. Y., and Jameson, D. J., 1956, Paleotectonic maps of the Jurassic system, with a separate section on Paleogeography by R. W. Imlay: U.S. Geol. Survey Misc. Geol. Inv. Map I-175, 6 p.

McKee, E. D., Oriel, S. S., Ketner, K. B., MacLachlan, M. E., Goldsmith, J. W., MacLachlan, J. C., and Mudge, M. R., 1959, Paleotectonic Maps of the Triassic System: U.S. Geol. Survey Misc. Geol. Inv. Map I-300, 33 p.

Mansfield, G. R., 1927, Geography, geology and mineral resources of southeastern Idaho: U.S. Geol. Survey Prof. Paper 152, 453 p.

Mapel, W. J., 1959, Geology and coal resources of the Buffalo-Lake DeSmet area, Johnson and Sheridan Counties, Wyoming: U.S. Geol. Survey Bull. 1078, 148 p. [1961].

Mapel, W. J., and Bergendahl, M. H., 1956, Gypsum Spring formation, northwestern Black Hills, Wyoming and South Dakota: Am. Assoc. Petroleum Geologists Bull., v. 40, no. 1, p. 84-93.

Maughan, E. K., 1967, Eastern Wyoming, eastern Montana and the Dakotas, Chap. G. in McKee, E. D., Oriel, S. S., and others, Paleotectonic investigations of the Permian System in the United States: U.S. Geol. Survey Prof. Paper 515, p. 125-152.

Merewether, E. A., 1972a, Geologic map of the Rawlins NW quadrangle, Carbon County, Wyoming: U.S. Geol. Survey Geol. Quad. Map GQ-1010.

1972b, Geologic map of the Seminoe Dam SW quadrangle, Carbon County, Wyoming: U.S. Geol Survey Geol. Quad. Map GQ-1017. 
Moench, R. H., and Schlee, J. S., 1967, Geology and uranium deposits of the Laguna district, New Mexico: U.S. Geol. Survey Prof. Paper 519, $117 \mathrm{p}$.

Mudge, M. R., 1972, Pre-Quaternary rocks in the Sun River Canyon area, northwestern Montana: U.S. Geol. Survey Prof. Paper 663-A, 142 p.

Muehlberger, W. R., Adams, G. E., Longgood, T. E., Jr., and St. John, B. E., 1960, Stratigraphy of the Chama quadrangle, northern Rio Arriba County, New Mexico, in Rio Chama Country: New Mexico Geol. Soc., 11th Field Conf. Guidebook, p. 93-102.

Nordquist, J. W., 1955, Pre-Rierdon Jurassic stratigraphy in northern Montana and Williston basin, in Billings Geol. Soc. Guidebook, 6th Ann. Field Conf., 1955, p. 96-106.

O'Sullivan, R. B., 1965, Geology of the Cedar Mesa-Boundary Butte area, San Juan County, Utah: U.S. Geol. Survey Bull. 1186, 128 p.

1970, The upper part of the Upper Triassic Chinle Formation and related rocks, southeastern Utah and adjacent areas: U.S. Geol. Survey Prof. Paper 644-E, 22 p.

1974, The Upper Triassic Chinle Formation in north-central New Mexico, in New Mexico Geol. Soc. Guidebook, 25th Ann. Field Conf., Central-northern New Mexico, 1974: p, 171-174.

O'Sullivan, R. B., and Beikman, H. M. (compilers), 1963, Geology, structure, and uranium deposits of the Shiprock quadrangle, New Mexico and Arizona: U.S. Geol. Survey Misc. Geol. Inv. Map I-345.

O'Sullivan, R. B., and Craig, L. C., 1973, Jurassic rocks of northeast Arizona and adjacent areas, in New Mexico Geol. Soc. Guidebook, 24th Field Conf., Monument Valley and vicinity, Arizona and Utah, 1973: p. 79-85.

O'Sullivan, R. B., and Green, M. W., 1973, Triassic rocks of northeast Arizona and adjacent areas, in New Mexico Geol. Soc. Guidebook, 24th Field Conf., Monument Valley and vicinity, Arizona and Utah, 1973: p. 72-78.

O'Sullivan, R. B., and MacLachlan, M. E., 1975, Triassic rocks of the Moab-White Canyon area, southeastern Utah, in Four Corners Geol. Soc. Guidebook 8th Field Conf., Canyonlands Country, 1975, p. 129-141.

O'Sullivan, R. B., Repenning, C. A., Beaumont, E. C., and Page, H. G., 1972, Stratigraphy of the Cretaceous rocks and the Tertiary Ojo Alamo Sandstone, Navajo and Hopi Indian Reservations, Arizona, New Mexico, and Utah: U.S. Geol. Survey Prof. Paper 521-E, 65 p.

Peck, R. E., 1957, North American Mesozoic Charophyta: U.S. Geol. Survey Prof. Paper 294-A, 44 p.

Peterson, Fred, 1973, Geologic map of the southwest quarter of the Gunsight Butte quadrangle, Kane and San Juan Counties, Utah, and Coconino County, Arizona: U.S. Geol. Survey Mineral Inv. Field Studies Map MF-306.

Peterson, Fred, and Barnum, B. E., 1973a, Geologic map of the southwest quarter of the Cummings Mesa quadrangle, Kane and San Juan Counties, Utah, and Coconino County, Arizona: U.S. Geol. Survey Misc. Geol. Inv. Map I-759.

1973b, Geologic map and coal resources of the northeast quarter of the Cummings Mesa quadrangle, Kane County, Utah: U.S. Geol. Survey Coal Inv. Map C-63.

Peterson, Fred, Cornet, Bruce, and Turner-Peterson, E. C., 1977, New data bearing on the stratigraphy and age of the Glen Canyon Group (Triassic and Jurassic) in southern Utah and northern Arizona: Geol. Soc. America Abs. with Programs, V. 9, no. 6, p. 755.

Peterson, Fred, and Pipiringos, G. N., 1978, Stratigraphic relations of the Navajo Sandstone to Middle Jurassic formations, southern Utah and northern Arizona: U.S. Geol. Survey Prof. Paper, 1035-B. In press.

Peterson, J. A., 1954, Marine Upper Jurassic, eastern Wyoming: Am. Assoc. Petroleum Geologists Bull., v. 38, no. 4, p. 463-507.

_1957, Marine Jurassic of northern Rocky Mountains and Williston Basin: Am. Assoc. Petroleum Geologists Bull., v. 41, no. 3, p. 399-440.
1972, Jurassic System, in Geologic atlas of the Rocky Mountain Region, United States of America: Rocky Mtn. Assoc. Geologists, Denver, Colorado, p. 177-189.

Phoenix, D. A., 1963, Geology of the Lees Ferry area, Coconino County, Arizona: U.S. Geol. Survey Bull. 1137, 86 p.

Pipiringos, G. N., 1957, Stratigraphy of the Sundance, Nugget, and Jelm Formations in the Laramie Basin, Wyoming: Wyoming Geol. Survey Bull. 47, 63 p.

1967, Jurassic and Triassic of Wyoming and southern Rockies [abs.]: Am. Assoc. Petroleum Geologists Bull., v. 51, no. 9, p. 1904-1905. 1968, Correlation and nomenclature of some Triassic and Jurassic rocks in south-central Wyoming: U.S. Geol. Survey Prof. Paper 594-D, 26 p. 1972, Upper Triassic and pre-Morrison Jurassic rocks, p. 18-29, in Segerstrom, Kenneth, and Young, E. J., 1972, General geology of the Hahns Peak and Farwell Mountain quadrangles, Routt County, Colorado: U.S. Geol. Survey Bull. 1349, 63 p.

Pipiringos, G. N., Hail, W. J., Jr., and Izett, G. A., 1969, The Chinle (Upper Triassic) and Sundance (Upper Jurassic) Formations in north-central Colorado: U.S. Geol. Survey Bull. 1274-N, p. N1-N35.

Pipiringos, G. N., and O'Sullivan, R. B., 1975, Chert pebble unconformity at the top of the Navajo Sandstone in southeastern Utah, in Four Corners Geol. Soc. Guidebook 8th Field Conf., Canyonlands Country, 1975: p. 149-156.

1976, Stratigraphic sections of some Triassic and Jurassic rocks from Douglas, Wyoming to Boulder, Colorado: U.S. Geol. Survey Oil and Gas Inv. Chart OC-69.

Post, E. V., 1967, Geology of the Cascade Springs quadrangle, Fall River County, South Dakota: U.S. Geol. Survey Bull. 1063-L, p. 443-504.

Rayl, R. L., 1956, Stratigraphy of the Nesson, Piper, and Rierdon Formations of central Montana, in Billings Geol. Soc. Guidebook, 7th Ann. Field Conf., 1956, p. 35-45.

Reeside, J. B., Jr., 1952, Summary of the stratigraphy of the Morrison Formation, in Yen, Teng-Chien, Molluscan fauna of the Morrison Formation: U.S. Geol. Survey Prof. Paper 233-B, p. 22-26.

Reeside, J. B., Jr., Chm., and others, 1957, Correlation of the Triassic Formations of North America, exclusive of Canada: Geol. Soc. America Bull., v. 68 , no. 11 , p. 1451-1514.

Repenning, C. A., Cooley, M. E., and Akers, J. P., 1969, Stratigraphy of the Chinle and Monekopi Formations, Navajo and Hopi Indian Reservations, Arizona, New Mexico, and Utah: U.S. Geol. Survey Prof. Paper 521-B, $34 \mathrm{p}$.

Reynolds, M. W., 1968, Geologic map of the Muddy Gap quadrangle, Carbon County, Wyoming: U.S. Geol. Survey Geol. Quad. Map GQ-771.

Richmond, G. M., 1945, Geology of northwest end of the Wind River Mountains, Sublette County, Wyoming: U.S. Geol. Survey Oil and Gas Inv. Prelim. Map 31.

Robinson, C. S., Mapel, W. J., and Bergendahl, M. H., 1964, Stratigraphy and structure of the northern and western flanks of the Black Hills uplift, Wyoming, Montana, and South Dakota: U.S. Geol. Survey Prof. Paper 404, $134 \mathrm{p}$.

Sandberg, D. T., 1959, Structure contour map on top of the middle member of the Piper formation of Middle Jurassic age in the Williston basin and adjacent areas in Montana, North Dakota, and South Dakota: U.S. Geol. Survey Oil and Gas Inv. Map OM-179.

Schell, E. M., and Yochelson, E. L., 1966, Permian-Triassic boundary in eastern Uintah County, Utah, and western Moffat County, Colorado, in Geological Survey research 1966: U.S. Geol. Survey Prof. Paper 550-D, p. D64-D68.

Shawe, D. R., Simmons, G. C., and Archbold, N. L., 1968, Stratigraphy of Slick Rock district and vicinity, San Miguel and Dolores Counties, Colorado: U.S. Geol. Survey Prof. Paper 576-A, p. Al-A108 [1969]. 
Sheldon, R. P., Cressman, E. R., Cheney, T. M., and McKelvey, V. E., 1967, Middle Rocky Mountains and northeastern Great Basin, Chap. H, in McKee, E. D., Oriel, S. S., and others, Paleotectonic investigations of the Permian System in the United States: U.S. Geol. Survey Prof. Paper 515 , p. 153-170.

Smith, J. F., Jr., Huff, L. C., Hinrichs, E. N., and Luedke, R. G., 1963, Geology of the Capitol Reef area, Wayne and Garfield Counties, Utah: U.S. Geol. Survey Prof. Paper 363, 102 p.

Staatz, M. H., and Albee, H. F., 1966, Geology of the Garns Mountain quadrangle, Bonneville, Madison, and Teton Counties, Idaho: U.S. Geol. Survey Bull. 1205, 122 p.

Stark, J. T., Johnson, J. H., Behre, C. H., Jr., Powers, W. E., Howland, A. L., Gould, D. B., and others, 1949, Geology and origin of South Park, Colorado: Geol. Soc. America Mem. 33, 188 p.

Stewart, J. H., Poole, F. G., and Wilson, R. F., 1972a, Stratigraphy and origin of the Chinle Formation and related Upper Triassic strata in the Colorado Plateau region, with a section on Sedimentary petrology, by $R$. A. Cadigan, and a section on Conglomerate studies, by William Thordarson, H. F. Albee, and J. H. Stewart: U.S. Geol. Survey Prof. Paper 690, $336 \mathrm{p}$.

_ 1972b, Stratigraphy and origin of the Triassic Moenkopi Formation and related strata in the Colorado Plateau region, with a section on Sedimentary petrology, by R. A. Cadigan: U.S. Geol. Survey Prof. Paper 691, $195 \mathrm{p}$.

Strobell, J. D., Jr., 1956, Geology of the Carrizo Mountains area in northeastern Arizona and northwestern New Mexico: U.S. Geol. Survey Oil and Gas Inv. Map OM-160.

Tohill, Bruce, and Picard, M. D., 1966, Stratigraphy and petrology of Crow Mountain Sandstone Member (Triassic), Chugwater Formation, northwestern Wyoming: Am. Assoc. Petroleum Geologists Bull., v. 50, no. 12 , p. $2547-2565$.

Tourtelot, H. A., 1953, Geology of the Badwater area, central Wyoming: U.S. Geol. Survey Oil and Gas Inv. Map OM-124.

U.S. Geological Survey, 1965, Geological research 1965: U.S. Geol. Survey Prof. Paper 525-A, p. A88. van Hinte, J. E., 1976, A Jurassic time scale: Am Assoc. Petroleum Geologists Bull., v. 60 , no. 4 , p. $489-497$.

Williams, P. L., (compiler), 1964, Geology, structure, and uranium deposits of the Moab quadrangle, Colorado and Utah: U.S. Geol. Survey Misc. Geol. Inv. Map I-360.

Wilson, R. F., and Stewart, J. H., 1967, Correlation of Upper Triassic and Triassic(?) formations between southwestern Utah and southern Nevada: U.S. Geol. Survey Bull. 1244-D, p. DI-D19.

Witkind, I. J., and Thaden, R. E., 1963, Geology and uranium-vanadium deposits of the Monument Valley area, Apache and Navajo Counties, Arizona, with sections on Serpentine at Garnet Ridge, by H. E. Malde and $\mathrm{R}$. E. Thaden, and Mineralogy and paragenesis of the ore deposits at the Monument No. 2 and Cato Sells mines, by D. H. Johnson: U.S. Geol. Survey Bull. 1103, 171 p.

Woodward, T. C., 1957, Gẻology of Deadman Butte area, Natrona County, Wyoming: Am. Assoc. Petroleum Geologists Bull., v. 41, no. 2, p. 212-262.

Wright, J. C., and Dickey, D. D., 1957, Entrada (San Rafael) studies [Colorado Plateau], in Geologic investigations of radioactive deposits semiannual progress report, Dec. 1, 1956-May 31, 1957: U.S. Geol. Survey TEI-690, book 2, p. 351-353.

_ 1958, Pre-Morrison Jurassic strata of southeastern Utah, in Inter-mountain Assoc. Petroleum Geologists, 9th Ann. Field Conf. Guidebook, p. 172-181.

_ 1963, Relations of the Navajo and Carmel formations in southwest Utah and adjoining Arizona, in Short papers in geology, hydrology, and topography: U.S. Geol. Survey Prof. Paper 450-E, p. E63-E67.

Wright, J. C., Shawe, D. R., and Lohman, S. W., 1962, Definition of members of Jurassic Entrada Sandstone in east-central Utah and west-central Colorado: Am. Assoc. Petroleum Geologists Bull., v. 46, no. 11, p. 2057-2070.

Zeller, H. D., and Stephens, E. V., 1973, Geologic map and coal resources of the Seep Flat quadrangle, Garfield and Kane Counties, Utah U.S. Geol. Survey Coal Inv. Map C-65 [1974].

Zieglar, D. L., 1956, Pre-Piper post-Minnekahta "red beds" in the Williston Basin, in North Dakota Geol. Soc., Williston Basin Symposium, Ist Internat., Bismarck, p. 170-178. 


